COMMUNICATIONS IN

ANALYSIS AND GEOMETRY

Volume 11, Number 4, 599-673, 2003

\title{
Convergence of spectral structures: a functional analytic theory and its applications to spectral geometry
}

\author{
Kazuhiro Kuwae and Takashi Shioya
}

\begin{abstract}
We present a functional analytic framework of some natural topologies on a given family of spectral structures on Hilbert spaces, and study convergence of Riemannian manifolds and their spectral structure induced from the Laplacian. We also consider convergence of Alexandrov spaces, locally finite graphs, and metric spaces with Dirichlet forms. Our study covers convergence of noncompact (or incomplete) spaces whose Laplacian has continuous spectrum.
\end{abstract}

\section{CONTENTS}

1. Introduction

2. Topologies on a family of spectral structures

2.1. Measured Gromov-Hausdorff topology

2.2. Convergence of Hilbert spaces

2.3. Convergence of bounded operators

2.4. Convergence of spectral measures on complex Hilbert spaces

2.5. Convergence of quadratic forms

2.6. Convergence of spectral structures

2.7. Asymptotic behavior of spectra

3. Convergence of manifolds

3.1. Preliminaries for Lipschitz-Riemannian manifold

3.2. Compact Lipschitz convergence and spectral structure

3.2.1. Blowing up

3.2.2. Magnifying

\footnotetext{
${ }^{1}$ The first author is partially supported by a Grant-in-Aid for Scientific Research No. 11740112 from the Ministry of Education, Science, Sports and Culture, Japan.

${ }^{2}$ The second author is partially supported by a Grant-in-Aid for Scientific Research No. 11440023, 14540056 from the Ministry of Education, Science, Sports and Culture, Japan.

${ }^{3}$ Dedicated to Professor Yukio Ogura on the occasion of his sixtieth birthday.
} 
3.2.3. Tower of coverings

3.2.4. Degeneration

3.3. Convergence of manifolds under a bound of local isoperimetric constant

3.3.1. $\quad$ Shrinking

3.3.2. Iteration of attaching small manifolds

3.4. Collapsing of warped product manifolds

3.5. Convergence of noncompact Alexandrov spaces

4. Convergence of graphs

4.1. Graph with simplicial metric

4.2. Convergence of graphs and spectral structure

4.3. Negligibility of boundary

5. Convergence of measured metric spaces with Dirichlet forms

5.1. Preliminaries for Dirichlet form

5.2. Asymptotic compactness of Dirichlet forms

5.3. Convergence of (noncompact) manifolds under a lower bound of Ricci curvature

\section{Introduction.}

The classical perturbation theory of linear operators tells us that if we perturb a Riemannian metric on a fixed manifold, then the spectral objects such as the spectral measure, the spectrum of the Laplacian etc. are continuous in metrics with respect to a suitable topology. What if we perturb not only the metric but also the topology of a manifold? In this case, there are no more natural identification between $L^{2}$ spaces of Riemannian manifolds and so we cannot rely on the standard perturbation theory. Nevertheless, we obtain some asymptotic correspondence between them under convergence of Riemannian volume measures. In this direction, Fukaya [23] first defined the measured Gromov-Hausdorff topology on the set of metric spaces with Radon measures (cf. $\S 2.1$ of this paper), and studied the convergence as $i \rightarrow \infty$ of the eigenvalues of the Laplacian of closed Riemannian manifolds $M_{i}, i=1,2, \ldots$, under a uniform bound of sectional curvature, when $M_{i}$ is convergent with respect to the measured Gromov-Hausdorff topology. After that, Kasue-Kumura [32, 33] (see also [7]) introduced a natural distance, called the spectral distance, between closed Riemannian manifolds under a uniform bound (in some sense) of heat kernel. The spectral distance expresses how close analytic structures are and is a powerful tool to study convergence of Riemannian manifolds and their analytic structure. In this paper, we present a systematic and functional analytic framework of some topologies on the set of spectral structures, which is much more general than the spectral distance. In particular, we do not need the existence of heat 
kernel (or the integral kernel of the semigroup), and also the spectrum is not needed to be discrete. Our framework is especially useful to investigate the behavior of spectrum of Laplacian under a perturbation of not only the metric but also the topology of (not necessarily compact) Riemannian manifolds.

We precisely mean by a spectral structure on a Hilbert space a compatible set $\Sigma=\left(A, \mathcal{E}, E,\left\{T_{t}\right\},\left\{R_{\zeta}\right\}\right)$ of a (nonnegative and selfadjoint) infinitesimal generator $A$, a closed nonnegative quadratic form $\mathcal{E}$, a spectral measure $E$, a strongly continuous contraction semigroup $\left\{T_{t}\right\}$, and a strongly continuous resolvent $\left\{R_{\zeta}\right\}$ on the Hilbert space. An important example of spectral structure is one whose generator is the Friedrichs extension of the $C^{\infty}$ Laplacian acting on the set, say $C_{0}^{\infty}(M)$, of smooth functions with compact support on a (not necessarily complete) Riemannian manifold $M$.

In what follows, let $(X, p)$ be any pointed, locally compact, and separable metric space and $m$ any Radon measure on it. We denote by $L^{2}(X ; m)$ the set of real valued $L^{2}$ functions with respect to $m$, equipped with $L^{2}$ inner product $(\cdot, \cdot)_{L^{2}(X ; m)}$. Then, this is a separable Hilbert space over $\mathbb{R}$. Consider the family $\mathcal{S}$ of all $(X, p, m, \Sigma)$, where $\Sigma$ is any spectral structure on $L^{2}(X ; m)$, and also the family $\mathcal{S}_{c}$ of $(X, p, m, \Sigma) \in \mathcal{S}$ for which $\Sigma$ has compact resolvent. In the first part $(\S 2)$ of this paper, we introduce a natural topology on $\mathcal{S}$ (resp. $\mathcal{S}_{c}$ ), called the strong (resp. compact) spectral topology (Definition 2.14), such that the projection $\mathcal{S}$ (resp. $\mathcal{S}_{c}$ ) $\ni(X, p, m, \Sigma) \mapsto(X, p, m)$ is continuous with respect to the pointed measured Gromov-Hausdorff topology on the family $\{(X, p, m)\}$. The convergence of spectral structures with respect to the strong (compact) spectral topology can be rephrased in terms of the quadratic forms, resolvent families, semigroups, and spectral measures respectively (Theorem 2.4). We investigate, when a sequence $\left(X_{i}, p_{i}, m_{i}, \Sigma_{i}\right) \in \mathcal{S}, i=1,2, \ldots$, converges with respect to the strong (compact) spectral topology, the asymptotic behavior of the spectrum $\sigma\left(A_{i}\right)$ of the generator $A_{i}$ of $\Sigma_{i}$ (Proposition 2.5 and Theorem 2.6). These results are generalizations of the classical topological perturbation theory (cf. [36, 47, 48]) and also of the recent study on convergence of bilinear forms, due to Mosco [42]. Remark here that even if $\left(X_{i}, p_{i}, m_{i}\right)$ for a large $i$ is close enough to $(X, p, m)$ with respect to the measured pointed Gromov-Hausdorff topology, we have no natural linear bijection between $L^{2}\left(X_{i} ; m_{i}\right)$ and $L^{2}(X ; m)$, and even no natural linear injection $L^{2}\left(X_{i} ; m_{i}\right) \rightarrow L^{2}(X ; m)$ or $L^{2}(X ; m) \rightarrow L^{2}\left(X_{i} ; m_{i}\right)$ for noncompact $X$. This makes some differences from the standard theory. Remark also that the concept of the compact spectral topology is newly defined and investigated in this paper. We indeed give the definitions of the strong and compact 
spectral topologies on the set of spectral structures on general Hilbert spaces, so that it can also be applied to that defined on $L^{2}$ differential forms, $L^{2}$ sections of vector bundles, $L^{2}$ functions on graphs, etc.

In the second part $(\S 3)$ of this paper, we apply the above functional analytic study on spectral structures to some sorts of shrinking, blowingup, and degenerating sequences of Riemannian manifolds. We define a new topology on the set of Riemannian manifolds, for which the sequences of manifolds as above are all convergent.

Definition 1.1 (Compact Lipschitz convergence). We say that a sequence $\left\{M_{i}\right\}_{i=1,2, \ldots}$ of Riemannian manifolds compact Lipschitz converges to a Riemannian manifold $M$ if for any relatively compact open subset $O \subset M$ there exists a sequence of relatively compact open subsets $O_{i} \subset M_{i}$ such that as $i \rightarrow \infty, O_{i}$ Lipschitz converges to $O$, i.e., the Lipschitz distance

$$
\begin{aligned}
d_{L}\left(O_{i}, O\right):= & \inf \left\{|\ln \operatorname{dil}(f)|+\left|\ln \operatorname{dil}\left(f^{-1}\right)\right|\right. \\
& \left.f: O_{i} \rightarrow O \text { is a bi-Lipschitz homeomorphism }\right\}
\end{aligned}
$$

tends to zero, where $\operatorname{dil}(f)$ is the smallest Lipschitz constant of $f$.

Note that compact Lipschitz convergence is only noncollapsing, i.e., preserving dimensions.

Let $M$ be a Riemannian manifold and $W^{1,2}(M)$ the $(1,2)$-Sobolev space of functions on $M$ (i.e., the set of real valued $L^{2}$ functions admitting $L^{2}$ weak derivative). Note that $W^{1,2}(M)$ is a real Hilbert space. Let $W_{0}^{1,2}(M)$ be the closure in $W^{1,2}(M)$ of $C_{0}^{\infty}(M)$. We denote by $\Sigma(M)$ the spectral structure on $L^{2}(M)$ induced from the Friedrichs extension, say $\Delta_{M}$, of the $C^{\infty}$ Laplacian acting on $C_{0}^{\infty}(M)$. Indicate by $\sigma(\cdot)$ the spectrum of an operator. The following theorem is a primitive application of our study on the spectral topologies.

Theorem 1.1. Let $\left\{M_{i}\right\}_{i=1,2, \ldots}$ be a sequence of (possibly noncompact, incomplete) Riemannian manifolds (with boundary), and $M$ a (possibly noncompact, incomplete) Riemannian manifold (with boundary) such that $W^{1,2}(M)=W_{0}^{1,2}(M)$. If $M_{i}$ compact Lipschitz converges to $M$, then the spectral structure $\Sigma\left(M_{i}\right)$ converges to $\Sigma(M)$ with respect to the strong spectral topology, and consequently we have

$$
\sigma\left(\Delta_{M}\right) \subset \lim _{i \rightarrow \infty} \sigma\left(\Delta_{M_{i}}\right),
$$

i.e., for any $\lambda \in \sigma\left(\Delta_{M}\right)$ there exist $\lambda_{i} \in \sigma\left(\Delta_{M_{i}}\right)$ with $\lambda_{i} \rightarrow \lambda$. 
Here, the condition $W_{0}^{1,2}(M)=W^{1,2}(M)$ is almost equivalent to the negligibility of the boundary in the sense of $[25,26,27]$. Note that in Theorem 1.1, the limit set of $\sigma\left(\Delta_{M_{i}}\right)$ does not necessarily coincides with $\sigma\left(\Delta_{M}\right)$ (Remark 3.2).

Although most of earlier studies treat only compact manifolds, there are some works for convergence $M_{i} \rightarrow M$ of complete noncompact manifolds: Colbois-Courtois [17] studied convergence of eigenvalues less than the infimum of essential spectrum of $M$, depending on control of the Dirichlet first eigenvalue around the ends of $M_{i}$. On the other hand, Theorem 1.1 says the semi-continuity of the whole spectrum. Also there are many works on degeneration of hyperbolic manifolds (see [56] for example), which is an example of compact Lipschitz convergence.

As a direct consequence of Theorem 1.1, we have an asymptotic estimate of the spectral gaps of a Riemannian manifold (Corollary 3.1). We extend Theorem 1.1 to collapsing of warped product manifolds (Theorem 3.3). Since the theorem is also true for Lipschitz-Riemannian manifolds, it can be applied to convergence of Alexandrov spaces (§3.5). We also study convergence of (locally finite) graphs $(\S 4)$, results which are analogous to compact Lipschitz convergence of manifolds.

In the final part $(\S 5)$ of this paper, we consider a family of measured metric spaces with (abstract) Dirichlet forms. Under some uniform condition on the metrics and measures, and a uniform bound of Poincaré constants, we prove the asymptotic compactness of the set of spectral structures associated with spaces in such a family (Theorems 5.1 and 5.2). Remark that we do not assume the doubling condition for the measures and also the existence of heat kernel (or the integral kernel of the semigroup). The results can be thought as generalizations of those due to Kasue-Kumura [32, 33]. Our proof is achieved in a different approach from theirs.

We have some geometric applications of that study. The first one is on convergence of compact Riemannian manifolds under a bound of isoperimetric constant. Let $M$ be an $n$-dimensional Riemannian manifold and $r>0$. An $r$-net of $M$ is defined to be a discrete (possibly finite) sequence $\left\{p_{k}\right\}_{k=1,2, \ldots}$ of points in $M$ with $\bigcup_{k} B\left(p_{k}, r\right)=M$, where $B(p, r)$ denotes the open metric ball centered at a point $p$ and of radius $r$. An $r$-net $\left\{p_{k}\right\}$ of $M$ is said to be $r$-discrete if $d\left(p_{k}, p_{\ell}\right) \geq r$ for all $k \neq \ell$, where $d$ is the distance function on $M$. We define the $r$-isoperimetric constant of $M$

$$
\mathcal{I}_{r}(M):=\sup _{\left\{p_{k}\right\}} \inf _{k, \Omega_{1}, \Omega_{2}} \frac{r \cdot \operatorname{area}\left(\partial \Omega_{1} \cap \partial \Omega_{2}\right)}{\operatorname{vol}\left(\Omega_{1}\right) \wedge \operatorname{vol}\left(\Omega_{2}\right)},
$$

where $\left\{p_{k}\right\}$ runs over all $r$-discrete $r$-nets of $M$ and $\left(\Omega_{1}, \Omega_{2}\right)$ over all pairs 
of two disjoint open domains in $B\left(p_{k}, r\right)$ with piecewise smooth boundary such that $\operatorname{vol}\left(B\left(p_{k}, r\right) \backslash\left(\Omega_{1} \cup \Omega_{2}\right)\right)=0$, and we set $x \wedge y:=\min \{x, y\}$ for $x, y \in \mathbb{R}$. For the completion $\bar{M}$ of an incomplete Riemannian manifold $M$, let us define a measure $\operatorname{vol}_{\bar{M}}$ on $\bar{M}$ to be the Riemannian volume measure $\operatorname{vol}_{M}$ on $M$ and to be zero on $\bar{M} \backslash M$. With these notation, we have:

Theorem 1.2. Let $\left\{M_{i}\right\}_{i=1,2, \ldots}$ be a sequence of compact Riemannian manifold (with boundary), and $M$ a (incomplete) Riemannian manifold (with boundary). We assume the following (1)-(5).

(1) $\overline{\lim }_{r \searrow 0} \underline{\lim }_{i \rightarrow \infty} \mathcal{I}_{r}\left(M_{i}\right)>0$.

(2) $W^{1,2}(M)=W_{0}^{1,2}(M)$.

(3) The completion $\bar{M}$ of $M$ is compact.

(4) $\left(M_{i}, \operatorname{vol}_{M_{i}}\right)$ converges to $\left(\bar{M}, \operatorname{vol}_{\bar{M}}\right)$ with respect to the measured Gromov-Hausdorff topology.

(5) $M_{i}$ compact Lipschitz converges to $M$.

Then, the spectral structure $\Sigma\left(M_{i}\right)$ converges to $\Sigma(M)$ with respect to the compact topology. Consequently, $\Sigma(M)$ has compact resolvent and for any fixed $k \in \mathbb{N}$, the $k^{\text {th }}$ eigenvalue of $\Delta_{M_{i}}$ converges to that of $\Delta_{M}$ as $i \rightarrow \infty$.

We construct an example of a sequence $\left\{M_{i}\right\}$ satisfying (1)-(5) by iterating to attach small manifolds. Such the manifold $M_{i}$ increases its topological complexity as $i \rightarrow \infty$ and the limit $M$ has infinite topological type (§3.3.2). In the same manner as in Theorem 3.3, it is possible to extend Theorem 1.2 to collapsing of warped product manifolds, which we omit to explore.

The second application is on convergence of complete noncompact Riemannian manifolds with a lower Ricci curvature bound. For a monotone nondecreasing function $c:[0, \infty) \rightarrow[0, \infty)$ and for a number $n \geq 2$, let $\mathcal{P} \mathcal{R}(n, c)$ be the set of $\left(M, p, \mu_{p}\right)$, where $(M, p)$ is any $n$-dimensional complete (possibly noncompact) pointed Riemannian manifold whose Ricci curvature satisfies $\operatorname{Ric}_{M} \geq-(n-1) c(R)$ on the metric ball $B(p, R)$ for every $R>0$, and we set $\mu_{p}:=\operatorname{vol}_{M} / \operatorname{vol}(B(p, 1))$. For $\left(M, p, \mu_{p}\right) \in \mathcal{P} \mathcal{R}(n, c)$, we define the $(1,2)$-Sobolev space $W^{1,2}\left(M ; \mu_{p}\right)$ to be the set of $u \in L^{2}\left(M ; \mu_{p}\right)$ admitting weak derivative $d u$ with $\int_{M}|d u|_{M}^{2} d \mu_{p}<\infty$, where $|\cdot|_{M}$ denotes the norm induced from the Riemannian metric on $M$. Let $\mathcal{E}_{\left(M, \mu_{p}\right)}$ : $W^{1,2}\left(M ; \mu_{p}\right) \times W^{1,2}\left(M ; \mu_{p}\right) \rightarrow \mathbb{R}$ be a Dirichlet form defined by

$$
\mathcal{E}_{\left(M, \mu_{p}\right)}(u, v):=\int_{M}\langle d u, d v\rangle_{M} d \mu_{p}, \quad u, v \in W^{1,2}\left(M ; \mu_{p}\right),
$$


and $\Sigma\left(M, \mu_{p}\right)$ the induced spectral structure on $L^{2}\left(M ; \mu_{p}\right)$. Cheeger-Colding [16] defined a canonical Dirichlet form, say $\mathcal{E}_{(X, m)}$, on $L^{2}(X ; m)$ for each measured pointed Gromov-Hausdorff limit $\left(X, p_{0}, m\right)$ of $\mathcal{P} \mathcal{R}(n, c)$. That induces a spectral structure on $L^{2}(X ; m)$, say $\Sigma(X, m)$. We have the following:

Theorem 1.3. Let a sequence $\left\{\left(M_{i}, p_{i}, \mu_{p_{i}}\right)\right\}_{i=1,2, \ldots} \subset \mathcal{P} \mathcal{R}(n, c)$ converge to a space $(X, p, m)$ with respect to the pointed measured Gromov-Hausdorff topology. Then, the spectral structure $\Sigma\left(M_{i}, \mu_{p_{i}}\right)$ converges to $\Sigma(X, m)$ with respect to the strong spectral topology. Consequently we have

$$
\sigma(\Delta) \subset \lim _{i} \sigma\left(\Delta_{i}\right)
$$

where $\Delta, \Delta_{i}$ are the generators of $\Sigma(X, m), \Sigma\left(M_{i}, \mu_{p_{i}}\right)$ respectively.

Note that we see from the discussions in $[32,16,15]$ that if $X$ is compact in Theorem 1.3, then $\Sigma\left(M_{i}, \mu_{p_{i}}\right)$ converges to $\Sigma(X, m)$ with respect to the compact spectral topology (see Remark 5.1).

Acknowledgment. The authors would like to thank Professors Atsushi Kasue, Yukio Ogura, Toshikazu Sunada, and Takao Yamaguchi for valuable discussions and comments.

\section{Topologies on a family of spectral structures.}

\subsection{Measured Gromov-Hausdorff topology •}

In this section, we shall define the (resp. measured) Gromov-Hausdorff topology on the set of pointed (resp. pointed and measured) metric spaces, which is originally defined by Gromov [28] (resp. Fukaya [23]). We establish some extensions of them for noncompact (or incomplete) spaces, and also present a natural definition of the $L^{2}$ topology over the set of $L^{2}$ functions on all locally compact separable measured metric spaces.

Throughout this paper, let $\mathcal{A}$ and $\mathcal{B}$ be any directed sets. Denote by $\mathcal{M}$ et the set of isometry classes of locally compact separable pointed metric spaces and by $\mathcal{M} e t_{c}$ the set of spaces in $\mathcal{M}$ et any bounded subset of which is relatively compact.

Definition 2.1 ((Compact) Gromov-Hausdorff topology). We say that a net $\left\{\left(X_{\alpha}, p_{\alpha}\right)\right\}_{\alpha \in \mathcal{A}}$ of spaces in Met converges to a space $(X, p) \in \mathcal{M}$ et in the sense of pointed compact Gromov-Hausdorff convergence (or compact $G H$ convergence) if and only if for any relatively compact open subset 
$O \subset X$ containing $p$, there exist positive numbers $\epsilon_{\alpha} \searrow 0$, relatively compact open subsets $O_{\alpha} \subset X_{\alpha}$ containing $p_{\alpha}$, and (not necessarily continuous) maps $f_{\alpha}:\left(O_{\alpha}, p_{\alpha}\right) \rightarrow(O, p), \alpha \in \mathcal{A}$, called $\epsilon_{\alpha}$-approximations, such that

$$
\begin{gathered}
\left|d\left(f_{\alpha}(x), f_{\alpha}(y)\right)-d_{\alpha}(x, y)\right|<\epsilon_{\alpha} \quad \text { for any } x, y \in O_{\alpha}, \\
O \subset B\left(f_{\alpha}\left(O_{\alpha}\right), \epsilon_{\alpha}\right),
\end{gathered}
$$

where $d_{\alpha}, d$ denote the distance functions on $X_{\alpha}, X$ respectively, and $B(A, r)$ the open metric $r$-ball of a set $A$ in a metric space. It follows from a straight forward discussion that this convergence induces a topology on $\mathcal{M}$ et, say the pointed compact Gromov-Hausdorff topology, or the compact GH topology for simplicity. We also define a stronger topology on $\mathcal{M e t}_{c}$, say the pointed Gromov-Hausdorff topology or the GH topology, by restricting $O=B(p, r)$ and $O_{\alpha}=B\left(p_{\alpha}, r_{\alpha}\right)$ in the above, where $r$ is any positive number and $\left\{r_{\alpha}\right\}$ a net of positive numbers depending on $r$ such that $r_{\alpha} \rightarrow r$.

Note that a notion of convergence of sequence of countable elements is not enough to define a topology and we need convergence of net for it.

The Gromov-Hausdorff topology on $\mathcal{M}$ et $t_{c}$ is originally defined in [28]. The compact Gromov-Hausdorff topology on $\mathcal{M}$ et introduced here is needed to cover the compact Lipschitz convergence of Riemannian manifolds.

Remark 2.1. (1) The restricted topology on $\mathcal{M e t}_{c}$ of the compact GH topology is different from (or strictly weaker than) the GH topology. In fact, setting $L_{a}:=\{(x, a) \mid x \in \mathbb{R}\} \subset \mathbb{R}^{2}, X:=L_{0}$, and $X_{\alpha}:=L_{0} \cup L_{1}$ for all $\alpha \in \mathcal{A}$ equipped with the restricted metrics of the Euclidean metric on $\mathbb{R}^{2}$, we have $\left(X_{\alpha}, o\right) \rightarrow(X, o)$ with respect to the compact GH topology, but not the GH topology.

(2) The GH topology on $\mathcal{M} e t_{c}$ is Hausdorff (see [28]). On the other hand, the compact GH topology on $\mathcal{M}$ et is not Hausdorff, as is observed in (1) above. In general, if a net $\left\{\left(X_{\alpha}, p_{\alpha}\right)\right\} \subset \mathcal{M}$ et compact GH converges to a space $(X, p) \in \mathcal{M}$ et, then it also compact $\mathrm{GH}$ converges to $(Y, p)$ for any open subset $Y \subset X$ containing $p$.

(3) Let a net $\left\{\left(X_{\alpha}, p_{\alpha}\right)\right\} \subset \mathcal{M}$ et compact GH converge to a space $(X, p) \in \mathcal{M}$ et. Then, a diagonal argument yields the existence of $\epsilon_{\alpha}$-approximations $f_{\alpha}:\left(O_{\alpha}, p_{\alpha}\right) \rightarrow\left(O_{\alpha}^{\prime}, p\right)$ with $\epsilon_{\alpha} \searrow 0$, where $\left\{O_{\alpha}^{\prime}\right\}$ is a monotone increasing net of relatively compact open subsets of $X$ containing $p$ and with $\bigcup_{\alpha} O_{\alpha}^{\prime}=X$, and $O_{\alpha} \subset X_{\alpha}$ relatively compact open subsets containing $p_{\alpha}$. 
Denote by $\mathcal{M}$ the set of isomorphism classes of triples $(X, p, m)$ with $(X, p) \in \mathcal{M}$ et and positive Radon measure $m$ on $X$, and by $\mathcal{M}_{c}$ the set of $(X, p, m) \in \mathcal{M}$ with $(X, p) \in \mathcal{M e t}_{c}$.

Definition 2.2 (Measured (and compact) Gromov-Hausdorff topology). We say that a net $\left\{\left(X_{\alpha}, p_{\alpha}, m_{\alpha}\right)\right\}_{\alpha \in \mathcal{A}}$ of spaces in $\mathcal{M}$ converges to a space $(X, p, m) \in \mathcal{M}$ in the sense of pointed, measured, and compact GromovHausdorff convergence (or measured compact GH) if and only if for any relatively compact open subset $O \subset X$ containing $p$, there exist positive numbers $\epsilon_{\alpha} \searrow 0$, relatively compact open subsets $O_{\alpha} \subset X_{\alpha}$ containing $p_{\alpha}$, and $m_{\alpha}$-measurable $\epsilon_{\alpha}$-approximations $f_{\alpha}:\left(O_{\alpha}, p_{\alpha}\right) \rightarrow(O, p)$ such that the push-forward measure $f_{\alpha *}\left(\left.m_{\alpha}\right|_{O_{\alpha}}\right)$ vaguely converges to $\left.m\right|_{O}$, i.e.,

$$
\lim _{\alpha} \int_{O_{\alpha}} u \circ f_{\alpha} d m_{\alpha}=\int_{O} u d m \quad \text { for any } u \in C_{0}(O),
$$

where $C_{0}(O)$ is the set of real valued continuous functions on $O$ with compact support in $O$. We call such a family of maps $\left\{f_{\alpha}\right\}$ a family of measured $\epsilon_{\alpha^{-}}$ approximations. This convergence induces a non-Hausdorff topology on $\mathcal{M}$, say the pointed, measured, and compact Gromov-Hausdorff topology, or the measured compact GH topology. Restricting $O=B(p, r)$ and $O_{\alpha}=B\left(p_{\alpha}, r_{\alpha}\right)$ for $r>0$ and $r_{\alpha} \rightarrow r$ in the above defines a stronger topology on $\mathcal{M}_{c}$, say the measured GH topology.

Remark 2.2. A measured compact GH convergence $\left(X_{\alpha}, p_{\alpha}, m_{\alpha}\right) \rightarrow$ $(X, p, m)$ is equivalent to the existence of $m_{\alpha}$-measurable $\epsilon_{\alpha}$-approximations $f_{\alpha}:\left(O_{\alpha}, p_{\alpha}\right) \rightarrow\left(O_{\alpha}^{\prime}, p\right), \epsilon_{\alpha} \searrow 0$, such that $\left\{O_{\alpha}^{\prime}\right\}$ is a monotone nondecreasing net of relatively compact open subsets of $X$ covering $X$ and that

$$
\lim _{\alpha} \int_{O_{\alpha}} u \circ f_{\alpha} d m_{\alpha}=\int_{X} u d m \quad \text { for any } u \in C_{0}(X) .
$$

This also holds for a measured GH convergence in $\mathcal{M}_{c}$ if we take $O_{\alpha}:=$ $B\left(p_{\alpha}, r_{\alpha}\right)$ and $O_{\alpha}^{\prime}:=B\left(p, r_{\alpha}^{\prime}\right)$ for some $r_{\alpha}, r_{\alpha}^{\prime} \nearrow \infty$ in the above. We also call such a family $\left\{f_{\alpha}\right\}$ a family of measured $\epsilon_{\alpha}$-approximations.

The measured GH topology was first introduced by Fukaya [23] on the set of compact metric spaces with Radon measures (see also $\S 3 \frac{1}{2}$ of [28]).

Note that if a sequence of Riemannian manifolds compact Lipschitz converges to a Riemannian manifold, then, by (3.1) in $\S 3.1$ below, this is also a convergence with respect to the measured compact GH topology defined here, by taking some reference points. For simplicity, we say (especially in 
$\S 3$ and $\S 4)$ that $\left(X_{\alpha}, m_{\alpha}\right)$ converges to $(X, m)$ with respect to the measured (compact) GH topology when $\left(X_{\alpha}, p_{\alpha}, m_{\alpha}\right)$ converges to $(X, p, m)$ for some reference points $p_{\alpha} \in X_{\alpha}$ and $p \in X$.

Proposition 2.1. The measured GH topology on $\mathcal{M}_{c}$ is Hausdorff.

Proof. Assume that a net $\left\{\left(X_{\alpha}, p_{\alpha}, m_{\alpha}\right)\right\}_{\alpha \in \mathcal{A}} \subset \mathcal{M}_{c}$ converges to two spaces $(X, p, m),\left(X^{\prime}, p^{\prime}, m^{\prime}\right) \in \mathcal{M}_{c}$. Then, there exist two measured $\epsilon_{\alpha}$-approximations $f_{\alpha}:\left(B\left(p_{\alpha}, r_{\alpha}\right), p_{\alpha}\right) \rightarrow\left(B\left(p, s_{\alpha}\right), p\right)$ and $g_{\alpha}$ : $\left(B\left(p_{\alpha}, r_{\alpha}^{\prime}\right), p_{\alpha}\right) \rightarrow\left(B\left(p^{\prime}, s_{\alpha}\right), p^{\prime}\right)$ with $\epsilon_{\alpha} \searrow 0, r_{\alpha}, r_{\alpha}^{\prime}, s_{\alpha} \nearrow \infty$. Here, taking a subnet if necessarily, we may assume that $r_{\alpha}^{\prime} \geq r_{\alpha}$ for all $\alpha \in \mathcal{A}$. By $(2.1)$ and $(2.2)$, we can construct $3 \epsilon_{\alpha}$-approximations $\hat{f}_{\alpha}:\left(B\left(p, s_{\alpha}\right), p\right) \rightarrow$ $\left(B\left(p_{\alpha}, r_{\alpha}\right), p_{\alpha}\right)$ such that $d\left(f_{\alpha} \circ \hat{f}_{\alpha}(x), x\right)<\epsilon_{\alpha}$ for any $x \in B\left(p, s_{\alpha}\right)$ and that $d\left(\hat{f}_{\alpha} \circ f_{\alpha}(x), x\right)<\epsilon_{\alpha}$ for any $x \in B\left(p_{\alpha}, r_{\alpha}\right)$. The maps $h_{\alpha}:=g_{\alpha} \circ \hat{f}_{\alpha}$ : $\left(B\left(p, s_{\alpha}\right), p\right) \rightarrow\left(B\left(p^{\prime}, s_{\alpha}\right), p^{\prime}\right)$ are $4 \epsilon_{\alpha}$-approximations and converge to an isometry $\iota:(X, p) \rightarrow\left(X^{\prime}, p^{\prime}\right)$ by taking a subnet (cf. 3.6 Proposition of [28]). We have

$$
\lim _{\alpha} \sup _{x \in B\left(p_{\alpha}, r_{\alpha}\right)} d\left(\iota \circ f_{\alpha}(x), g_{\alpha}(x)\right)=0 .
$$

Let $u \in C_{0}\left(X^{\prime}\right)$ be any fixed function. The uniform continuity of $u$ and (2.3) together show that for any $\epsilon>0$ there exists $\alpha_{\epsilon} \in \mathcal{A}$ such that $\mid u \circ \iota \circ f_{\alpha}(x)-$ $u \circ g_{\alpha}(x) \mid<\epsilon$ for any $\alpha \geq \alpha_{\epsilon}$ and $x \in B\left(p_{\alpha}, r_{\alpha}\right)$. Therefore,

$$
\begin{aligned}
& \varlimsup_{\alpha}\left|\int_{B\left(p_{\alpha}, r_{\alpha}\right)} u \circ \iota \circ f_{\alpha} d m_{\alpha}-\int_{B\left(p_{\alpha}, r_{\alpha}\right)} u \circ g_{\alpha} d m_{\alpha}\right| \\
& \leq \epsilon \varlimsup_{\alpha} m_{\alpha}\left(\left(\iota \circ f_{\alpha}\right)^{-1}(\operatorname{supp} u) \cup g_{\alpha}^{-1}(\operatorname{supp} u)\right) \leq \epsilon m(B(\operatorname{supp} u, \epsilon)) .
\end{aligned}
$$

Since supp $u \subset B\left(p^{\prime}, s_{\alpha}\right)$ for all sufficiently large $\alpha$, we have

$$
\begin{aligned}
\int_{X^{\prime}} u \circ \iota d m & =\lim _{\alpha} \int_{B\left(p_{\alpha}, r_{\alpha}\right)} u \circ \iota \circ f_{\alpha} d m_{\alpha} \\
& =\lim _{\alpha} \int_{B\left(p_{\alpha}, r_{\alpha}\right)} u \circ g_{\alpha} d m_{\alpha}=\int_{X^{\prime}} u d m^{\prime},
\end{aligned}
$$

which means that $\iota_{*} m=m^{\prime}$. Thus, $(X, p, m)$ and $\left(X^{\prime}, p^{\prime}, m^{\prime}\right)$ are isomorphic as pointed measured metric space. This completes the proof.

Let $h:[0, \infty) \rightarrow[0, \infty)$ be a monotone nondecreasing function, and set

$$
\mathcal{M}_{c}(h):=\left\{(X, p, m) \in \mathcal{M}_{c} \mid m(B(p, r)) \leq h(r) \text { for all } r>0\right\} .
$$


Proposition 2.2. The projection $\mathcal{M}_{c}(h) \ni(X, p, m) \mapsto(X, p) \in \mathcal{M e t}_{c}$ is a proper map.

Proof. Let $\left\{\left(X_{\alpha}, p_{\alpha}, m_{\alpha}\right)\right\}_{\alpha \in \mathcal{A}}$ be a net of spaces in $\mathcal{M}_{c}(h)$ such that $\left(X_{\alpha}, p_{\alpha}\right)$ $\mathrm{GH}$ converges to some space $(X, p) \in \mathcal{M e t}_{c}$. It suffices to prove that there exists a Radon measure $m$ on $X$ with $(X, p, m) \in \mathcal{M}_{c}(h)$ such that some subnet of $\left\{\left(X_{\alpha}, p_{\alpha}, m_{\alpha}\right)\right\}$ converges to $(X, p, m)$ with respect to the measured GH topology. We shall prove that $\epsilon_{\alpha}$-approximations $f_{\alpha}:\left(B\left(p_{\alpha}, r_{\alpha}\right), p_{\alpha}\right) \rightarrow$

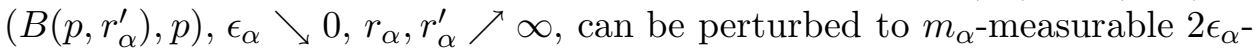
approximations. In fact, we first perturb them to satisfy that their images are discrete, which is easy to be done. We in addition perturb them to ones such that the preimage of each point in $X$ is a Borel subset of $X_{\alpha}$, which can be done by dividing $X_{\alpha}$ into disjoint Borel subsets of small diameters. Then, the perturbed approximations are $m_{\alpha}$-measurable. We may thus assume that the approximations $f_{\alpha}$ are $m_{\alpha}$-measurable from the beginning. It is easy to see that for any $r>0$ and $\alpha \in \mathcal{A}$ with $r_{\alpha}^{\prime} \geq r$, the push-forward measure $m_{\alpha, r}:=\left(\left.f_{\alpha_{*}}\left(m_{\alpha} \mid B\left(p_{\alpha}, r_{\alpha}\right)\right)\right|_{B(p, r)}\right.$ is a Radon measure on $B(p, r)$ with $\varlimsup_{\alpha} m_{\alpha, r}(B(p, r)) \leq h(r)$. We take a sequence $r(i) \nearrow \infty, i=1,2, \ldots$ By taking a subnet and by a diagonal argument, for each fixed $i$ the measure $m_{\alpha, r(i)}$ vaguely converges to a Radon measure $m_{r(i)}$ on $B(p, r(i))$ with total measure $\leq h(r(i))$ and with the property that $\left.m_{r(i+1)}\right|_{B(p, r(i))}=m_{r(i)}$. The inductive limit, say $m$, of $m_{r(i)}$ as $i \rightarrow \infty$ is a Radon measure on $X$, so that in particular $(X, p, m) \in \mathcal{M}_{c}$. It is obvious that $\left(X_{\alpha}, p_{\alpha}, m_{\alpha}\right)$ converges to $(X, p, m)$ with respect to the measured GH topology. This completes the proof.

The following is a direct consequence of the proposition.

Corollary 2.1. Let $\mathcal{C}$ be a subset of $\mathcal{M}_{c}(h)$ such that $\{(X, p) \mid(X, p, m) \in$ $\mathcal{C}\}$ is $G H$ relatively compact in $\mathcal{M e t}_{c}$. Then, $\mathcal{C}$ is measured $G H$ relatively compact in $\mathcal{M}_{c}$.

For $(X, p, m) \in \mathcal{M}$ and $K=\mathbb{R}$ or $\mathbb{C}$, we denote by $C_{0}(X, K)$ the set of $K$-valued continuous functions on $X$ with compact support in $X$, and by $L^{2}(X, K ; m)$ the set of $K$-valued $L^{2}$ functions on $X$ with respect to $m$ with $L^{2}$ inner product defined by

$$
(u, v)_{L^{2}}:=(u, v)_{L^{2}(X, K ; m)}:=\int_{X} u \bar{v} d m, \quad u, v \in L^{2}(X, K ; m) .
$$


Define the norm $\|u\|_{L^{2}}:=\|u\|_{L^{2}(X, K ; m)}:=\sqrt{(u, u)_{L^{2}(X, K ; m)}}$. It follows that $L^{2}(X, K ; m)$ is a separable Hilbert space over $K$. Note that $C_{0}(\operatorname{supp} m, K)$ is densely embedded into $L^{2}(X, K ; m)$ and that $C_{0}(X, K)$ is not embedded into $L^{2}(X, K ; m)$ if $\operatorname{supp} m \neq X$. We sometimes omit $K$ and write $L^{2}(X ; m)$ and $C_{0}(X)$ only when $K=\mathbb{R}$.

Definition 2.3 ( $L^{2}$ topology). Let $\left\{\left(X_{\alpha}, p_{\alpha}, m_{\alpha}\right)\right\}_{\alpha \in \mathcal{A}}$ be a net of spaces in $\mathcal{M}$ and $(X, p, m) \in \mathcal{M}$ be a space. A net $\left\{u_{\alpha}\right\}_{\alpha \in \mathcal{A}}$ of functions with $u_{\alpha} \in L^{2}\left(X_{\alpha}, K ; m_{\alpha}\right)$ is said to (strongly) $L^{2}$ converges to a function $u \in L^{2}(X, K ; m)$ if $\left(X_{\alpha}, p_{\alpha}, m_{\alpha}\right)$ converges to $(X, p, m)$ with respect to the measured compact GH topology and if there exists a net $\left\{\tilde{u}_{\beta}\right\}_{\beta \in \mathcal{B}}$ of functions in $C_{0}(\operatorname{supp} m, K)$ tending to $u$ in $L^{2}(X, K ; m)$ such that

$$
\lim _{\beta} \varlimsup_{\alpha}\left\|\Phi_{\alpha} \tilde{u}_{\beta}-u_{\alpha}\right\|_{L^{2}\left(X_{\alpha}, K ; m_{\alpha}\right)}=0
$$

where $f_{\alpha}:\left(O_{\alpha}, p_{\alpha}\right) \rightarrow\left(O_{\alpha}^{\prime}, p\right)$ are $\epsilon_{\alpha}$-approximations with $\bigcup_{\alpha} O_{\alpha}^{\prime}=X$ and $\epsilon_{\alpha} \searrow 0$, and where for $v \in C_{0}(\operatorname{supp} m, K), \Phi_{\alpha} v:=v \circ f_{\alpha}$ on $O_{\alpha}$ and $\Phi_{\alpha} v:=0$ on $X_{\alpha} \backslash O_{\alpha}$. This convergence defines a topology, say the (strong) $L^{2}$ topology, on the disjoint union

$$
L^{2}(\mathcal{M}, K):=\bigsqcup_{(X, p, m) \in \mathcal{M}} L^{2}(X, K ; m) .
$$

We also define the $L^{2}$ topology on $L^{2}\left(\mathcal{M}_{c}, K\right)$ in the same manner by using the measured GH topology on $\mathcal{M}_{c}$. We sometimes omit $K$ and write $L^{2}(\mathcal{M})$ and $L^{2}\left(\mathcal{M}_{c}\right)$ if $K=\mathbb{R}$.

We could consider $L^{2}(\mathcal{M}, K)$ (resp. $\left.L^{2}\left(\mathcal{M}_{c}, K\right)\right)$ as a Hilbert space bundle over $\mathcal{M}$ (resp. $\left.\mathcal{M}_{c}\right)$. It follows that the natural projections $L^{2}(\mathcal{M}, K) \rightarrow \mathcal{M}$ and $L^{2}\left(\mathcal{M}_{c}, K\right) \rightarrow \mathcal{M}_{c}$ are continuous. Obviously, the inclusion map $L^{2}\left(\mathcal{M}_{c}, K\right) \rightarrow L^{2}(\mathcal{M}, K)$ is continuous. Proposition 2.1 implies the Hausdorff property of $L^{2}\left(\mathcal{M}_{c}, K\right)$ (see Corollary 2.2 below for the precise proof in a more general setting). On the other hand, $L^{2}(\mathcal{M}, K)$ is not Hausdorff because $\mathcal{M}$ is not Hausdorff.

We can extend Definition 2.3 for the space of $L^{2}$ differential forms on manifolds and also for the space of $L^{2}$ sections on vector bundles based on compact Lipschitz convergence. By this reason, it is most convenient to generalize it for general Hilbert spaces by forgetting base spaces. 


\subsection{Convergence of Hilbert spaces.}

Let $K:=\mathbb{R}$ or $\mathbb{C}$ be fixed. For any Hilbert space $H$ over $K$, we denote the inner product by $(\cdot, \cdot)_{H}$ and the norm by $\|\cdot\|_{H}$. Here we assume that $u \mapsto$ $(u, v)_{H}$ is linear and $(u, v)_{H}=\overline{(v, u)_{H}}$ for any $u, v \in H$ when $K=\mathbb{C}$. Assume also that all Hilbert spaces in this paper are separable. Let us give a family $\left\{H_{\nu}\right\}_{\nu \in \mathcal{N}}$ of Hilbert spaces over $K$ and a family $\left\{\Phi_{\nu, \mu}: \mathcal{C}_{\nu} \rightarrow H_{\mu}\right\}_{\nu, \mu \in \mathcal{N}}$ of linear maps with dense domains $\mathcal{C}_{\nu} \subset H_{\nu}$ such that $\Phi_{\nu, \nu}$ for each $\nu \in \mathcal{N}$ is the identity operator on $\mathcal{C}_{\nu}$. Assume that $\left\{H_{\nu}\right\}_{\nu \in \mathcal{N}}$ (i.e., $\mathcal{N}$ ) has a (not necessarily Hausdorff) topology such that a net $\left\{H_{\nu_{\alpha}}\right\}_{\alpha \in \mathcal{A}}$ converges to an $H_{\nu}$ if and only if, setting

$$
H_{\alpha}:=H_{\nu_{\alpha}}, \quad H:=H_{\nu}, \quad \Phi_{\alpha}:=\Phi_{\nu, \nu_{\alpha}}, \quad \mathcal{C}:=\mathcal{C}_{\nu}
$$

for simplicity, we have

$$
\lim _{\alpha}\left\|\Phi_{\alpha} u\right\|_{H_{\alpha}}=\|u\|_{H} \quad \text { for any } u \in \mathcal{C} .
$$

Note that $\Phi_{\alpha}$ is asymptotically close to a unitary operator, however it is not necessarily injective even for sufficiently large $\alpha$. Throughout this and later sections, we suppose:

Assumption 2.1. A net $\left\{H_{\alpha}\right\}_{\alpha \in \mathcal{A}}$ converges to an $H$.

Definition 2.4 (Strong topology on $\mathcal{H}$ ). We say that a net $\left\{u_{\alpha}\right\}_{\alpha \in \mathcal{A}}$ with $u_{\alpha} \in H_{\alpha}$ (strongly) converges to a vector $u \in H$ if there exists a net $\left\{\tilde{u}_{\beta}\right\}_{\beta \in \mathcal{B}} \subset \mathcal{C}$ tending to $u$ in $H$ such that

$$
\lim _{\beta} \varlimsup_{\alpha}\left\|\Phi_{\alpha} \tilde{u}_{\beta}-u_{\alpha}\right\|_{H_{\alpha}}=0 .
$$

We call the topology on the disjoint union $\mathcal{H}:=\bigsqcup_{\nu \in \mathcal{N}} H_{\nu}$ induced from this convergence the strong topology on $\mathcal{H}$.

We define a topology on the family

$$
\left\{L^{2}(X ; m)\right\}_{(X, p, m) \in \mathcal{M}_{c}} \quad\left(\text { resp. } \quad\left\{L^{2}(X ; m)\right\}_{(X, p, m) \in \mathcal{M})}\right.
$$

by the measured (resp. compact and measured) GH topology on $\mathcal{M}_{c}$ (resp. $\mathcal{M})$. Then, $\mathcal{H}$ is seen to be a generalization of $L^{2}(\mathcal{M}, K)$ and $L^{2}\left(\mathcal{M}_{c}, K\right)$.

Lemma 2.1. Let $\left\{u_{\alpha}\right\}_{\alpha \in \mathcal{A}},\left\{v_{\alpha}\right\}_{\alpha \in \mathcal{A}}$ be two nets of vectors in $\mathcal{H}$ with $u_{\alpha}, v_{\alpha} \in H_{\alpha}$, and let $u, v \in H$. Then, we have the following: 
(1) $u_{\alpha} \rightarrow 0 \in H$ in $\mathcal{H}$ if and only if $\left\|u_{\alpha}\right\|_{H_{\alpha}} \rightarrow 0$.

(2) If $u_{\alpha} \rightarrow u$ in $\mathcal{H}$, then $\left\|u_{\alpha}\right\|_{H_{\alpha}} \rightarrow\|u\|_{H}$.

(3) If $u_{\alpha} \rightarrow u$ and $v_{\alpha} \rightarrow v$ in $\mathcal{H}$, then $a u_{\alpha}+b v_{\alpha} \rightarrow a u+b v$ in $\mathcal{H}$ for any $a, b \in K$.

(4) If $u_{\alpha} \rightarrow u$ and $v_{\alpha} \rightarrow v$ in $\mathcal{H}$, then $\left(u_{\alpha}, v_{\alpha}\right)_{H_{\alpha}} \rightarrow(u, v)_{H}$.

(5) If $\left\|u_{\alpha}-v_{\alpha}\right\|_{H_{\alpha}} \rightarrow 0$ and $u_{\alpha} \rightarrow u$ in $\mathcal{H}$, then $v_{\alpha} \rightarrow u$ in $\mathcal{H}$.

(6) If $u_{\alpha} \rightarrow u$ and $v_{\alpha} \rightarrow u$ in $\mathcal{H}$, then $\left\|u_{\alpha}-v_{\alpha}\right\|_{H_{\alpha}} \rightarrow 0$.

(7) For any $w \in H$ there exists a net $\left\{w_{\alpha}\right\}_{\alpha \in \mathcal{A}}$ with $w_{\alpha} \in H_{\alpha}$ which converges to $w$ in $\mathcal{H}$.

Proof. We prove only (6), because the proofs of the others are easy. Assume that $u_{\alpha} \rightarrow u$ and $v_{\alpha} \rightarrow u$ in $\mathcal{H}$. Then, there are $\tilde{u}_{\alpha}, \tilde{v}_{\alpha} \in \mathcal{C}$ both tending to $u$ in $H$ such that

$$
\lim _{\beta} \varlimsup_{\alpha}\left\|\Phi_{\alpha} \tilde{u}_{\beta}-u_{\alpha}\right\|_{H_{\alpha}}=\lim _{\beta} \varlimsup_{\alpha}\left\|\Phi_{\alpha} \tilde{v}_{\beta}-v_{\alpha}\right\|_{H_{\alpha}}=0 .
$$

It follows that

$$
\begin{aligned}
\lim _{\beta} \varlimsup_{\alpha}\left\|\Phi_{\alpha} \tilde{u}_{\beta}-\Phi_{\alpha} \tilde{v}_{\beta}\right\|_{H_{\alpha}} & =\lim _{\beta} \varlimsup_{\alpha}\left\|\Phi_{\alpha}\left(\tilde{u}_{\beta}-\tilde{v}_{\beta}\right)\right\|_{H_{\alpha}} \\
& =\lim _{\beta}\left\|\tilde{u}_{\beta}-\tilde{v}_{\beta}\right\|_{H}=0,
\end{aligned}
$$

which together with (2.4) completes the proof.

Corollary 2.2. The strong topology on $\mathcal{H}$ is Hausdorff if and only if $\left\{H_{\nu}\right\}_{\nu \in \mathcal{N}}$ is Hausdorff.

Proof. It is obvious that the Hausdorff property of $\left\{H_{\nu}\right\}_{\nu \in \mathcal{N}}$ implies that of the strong topology on $\mathcal{H}$. Let us prove the converse. We assume that $\left\{H_{\nu}\right\}_{\nu \in \mathcal{N}}$ is Hausdorff, so that the limit of a convergent net $\left\{H_{\alpha}\right\}_{\alpha \in \mathcal{A}} \subset$ $\left\{H_{\nu}\right\}_{\nu \in \mathcal{N}}$ is only one. Under Assumption 2.1 we suppose that a net $u_{\alpha} \in H_{\alpha}$ strongly converges to two vectors $u, v \in H$. Then, Lemma 2.1(3) implies that $0=u_{\alpha}-u_{\alpha} \in H_{\alpha}$ converges to $u-v$. By Lemma 2.1(2), we have $\|u-v\|_{H}=0$. This completes the proof. 
Definition 2.5 (Weak topology on $\mathcal{H}$ ). We say that a net $\left\{u_{\alpha}\right\}_{\alpha \in \mathcal{A}}$ with $u_{\alpha} \in H_{\alpha}$ weakly converges to vector $u \in H$ if

$$
\lim _{\alpha}\left(u_{\alpha}, v_{\alpha}\right)_{H_{\alpha}}=(u, v)_{H}
$$

for any net $\left\{v_{\alpha}\right\}_{\alpha \in \mathcal{A}}$ with $v_{\alpha} \in H_{\alpha}$ tending to a $v \in H$ in $\mathcal{H}$. This convergence induces a topology on $\mathcal{H}$, say the weak topology on $\mathcal{H}$.

It is easy to see that the weak topology on $\mathcal{H}$ is Hausdorff if and only if so is $\left\{H_{\nu}\right\}_{\nu \in \mathcal{N}}$. By Lemma 2.1(4), a strong convergence implies the weak convergence, so that the strong topology on $\mathcal{H}$ is stronger than the weak topology on $\mathcal{H}$.

Lemma 2.2. Let $\left\{u_{\alpha}\right\}_{\alpha \in \mathcal{A}}$ be a net with $u_{\alpha} \in H_{\alpha}$. If $\left\|u_{\alpha}\right\|_{H_{\alpha}}$ is uniformly bounded for $\alpha \in \mathcal{A}$, there exists a weakly convergent subnet of $\left\{u_{\alpha}\right\}_{\alpha \in \mathcal{A}}$.

Proof. The proof is little more delicate than the standard. Let $\left\{\varphi_{k}\right\}_{k \in \mathbb{N}}$ be a complete orthonormal basis of $H$. For each $k$ there is a net $\left\{\tilde{\varphi}_{k, \beta}\right\}_{\beta \in \mathcal{B}} \subset \mathcal{C}$ such that $\lim _{\beta} \tilde{\varphi}_{k, \beta}=\varphi_{k}$ in $H$. Replacing with subnets of $\mathcal{A}$ and $\mathcal{B}$ if necessarily, we assume that the limit

$$
\lim _{\beta} \lim _{\alpha}\left(u_{\alpha}, \Phi_{\alpha} \tilde{\varphi}_{1, \beta}\right)_{H_{\alpha}}=: a_{1} \in \bar{K}
$$

exists, where $\bar{K}$ denotes the one-point compactification of $K$. Here, it follows from the uniform boundedness of $\left\|u_{\alpha}\right\|_{H_{\alpha}}$ that $a_{1} \in K$. Repeating this procedure, we may assume that

$$
\lim _{\beta} \lim _{\alpha}\left(u_{\alpha}, \Phi_{\alpha} \tilde{\varphi}_{k, \beta}\right)_{H_{\alpha}}=: a_{k} \in K
$$

exists for every $k \in \mathbb{N}$. Let us fix a number $N \in \mathbb{N}$ for a while. For any $\epsilon>0$ there is a $\beta_{\epsilon} \in \mathcal{B}$ such that $\left|\left(\tilde{\varphi}_{k, \beta}, \tilde{\varphi}_{l, \beta}\right)_{H}-\delta_{k l}\right|<\epsilon$ for any $k, l=$ $1, \ldots, N$ and $\beta \geq \beta_{\epsilon}$. Moreover, for any $\beta \geq \beta_{\epsilon}$ there is an $\alpha_{\epsilon, \beta} \in \mathcal{A}$ such that $\left|\left(\Phi_{\alpha} \tilde{\varphi}_{k, \beta}, \Phi_{\alpha} \tilde{\varphi}_{l, \beta}\right)_{H_{\alpha}}-\delta_{k l}\right|<2 \epsilon$ for any $k, l=1, \ldots, N$ and $\alpha \geq \alpha_{\epsilon, \beta}$. Therefore, setting $L_{\alpha, \beta}:=\left\langle\Phi_{\alpha} \tilde{\varphi}_{k, \beta}\right\rangle_{k=1, \ldots, N}$ (linear span), we have

$$
\left.\left|\sum_{k=1}^{N}\right|\left(u_{\alpha}, \Phi_{\alpha} \tilde{\varphi}_{k, \beta}\right)_{H_{\alpha}}\right|^{2}-\left\|P_{L_{\alpha, \beta}} u_{\alpha}\right\|_{H_{\alpha}}^{2} \mid<\theta_{N}(\epsilon)
$$

for any $\alpha \geq \alpha_{\epsilon, \beta}$ and $\beta \geq \beta_{\epsilon}$, where $P_{L}: H_{\alpha} \rightarrow L$ denotes the projection to a linear subspace $L \subset H_{\alpha}$ and $\theta_{N}(\cdot)$ some function depending only on $N$ 
such that $\lim _{\epsilon \rightarrow 0} \theta_{N}(\epsilon)=0$. This implies

$$
\begin{aligned}
\sum_{k=1}^{N}\left|a_{k}\right|^{2} & =\lim _{\beta} \lim _{\alpha} \sum_{k=1}^{N}\left|\left(u_{\alpha}, \Phi_{\alpha} \tilde{\varphi}_{k, \beta}\right)_{H_{\alpha}}\right|^{2}=\lim _{\beta} \lim _{\alpha}\left\|P_{L_{\alpha, \beta}} u_{\alpha}\right\|_{H_{\alpha}}^{2} \\
& \leq \varlimsup_{\alpha}\left\|u_{\alpha}\right\|_{H_{\alpha}}^{2}<\infty
\end{aligned}
$$

for any $N \in \mathbb{N}$, so that

$$
u:=\sum_{k=1}^{\infty} a_{k} \varphi_{k} \in H
$$

We shall prove that some subnet of $\left\{u_{\alpha}\right\}_{\alpha \in \mathcal{A}}$ weakly converges to $u$. Take any $v \in H$ and set $b_{k}:=\left(v, \varphi_{k}\right)_{H}$. By Lemma 2.1(6), it suffices to show that (2.5) holds for some net $\left\{v_{\alpha}\right\}_{\alpha \in \mathcal{A}}$. Let $\tilde{v}_{\beta}^{N}:=\sum_{k=1}^{N} b_{k} \tilde{\varphi}_{k, \beta}$. Clearly, $\tilde{v}_{\beta}^{N} \in \mathcal{C}$ and $\lim _{N \rightarrow \infty} \lim _{\beta} \tilde{v}_{\beta}^{N}=v$ (strongly). We have

$$
\lim _{\beta} \lim _{\alpha}\left(u_{\alpha}, \Phi_{\alpha} \tilde{v}_{\beta}^{N}\right)_{H_{\alpha}}=\lim _{\beta} \lim _{\alpha} \sum_{k=1}^{N} b_{k}\left(u_{\alpha}, \Phi_{\alpha} \tilde{\varphi}_{k, \beta}\right)_{H_{\alpha}}=\sum_{k=1}^{N} a_{k} b_{k},
$$

which tends to $(u, v)_{H}$ as $N \rightarrow \infty$. Thus, there exists a net $\left\{N_{\beta}\right\}_{\beta \in \mathcal{B}}$ of natural numbers (slowly) tending to $\infty$ such that $\lim _{\beta} \tilde{v}_{\beta}^{N_{\beta}}=v$ (strongly) and

$$
\lim _{\beta} \lim _{\alpha}\left(u_{\alpha}, \Phi_{\alpha} \tilde{v}_{\beta}^{N_{\beta}}\right)_{H_{\alpha}}=(u, v)_{H} .
$$

This completes the proof.

Lemma 2.3. Let $\left\{u_{\alpha}\right\}_{\alpha \in \mathcal{A}}$ be a net with $u_{\alpha} \in H_{\alpha}$ weakly converging to a vector $u \in H$. Then we have

$$
\sup _{\alpha}\left\|u_{\alpha}\right\|_{H_{\alpha}}<\infty \quad \text { and } \quad\|u\|_{H} \leq \frac{\lim }{\alpha}\left\|u_{\alpha}\right\|_{H_{\alpha}} .
$$

Moreover, $u_{\alpha} \rightarrow u$ strongly if and only if

$$
\|u\|_{H}=\lim _{\alpha}\left\|u_{\alpha}\right\|_{H_{\alpha}} .
$$

Proof. Suppose $\sup _{\alpha}\left\|u_{\alpha}\right\|=\infty$. Then, there is a countable subnet $\left\{u_{\alpha_{k}}\right\}_{k=1}^{\infty}$ of $\left\{u_{\alpha}\right\}$ such that $\left\|u_{\alpha_{k}}\right\|_{H_{\alpha_{k}}} \geq k$. Setting

$$
v_{k}:=\frac{1}{k} \cdot \frac{u_{\alpha_{k}}}{\left\|u_{\alpha_{k}}\right\|_{H_{\alpha_{k}}}}
$$


one has $\left\|v_{k}\right\|_{H_{\alpha_{k}}}=1 / k \rightarrow 0$ and hence $v_{k} \rightarrow 0$ in $\mathcal{H}$, which implies $\left(u_{\alpha_{k}}, v_{k}\right)_{H_{\alpha_{k}}} \rightarrow(u, 0)_{H}=0$. On the other hand,

$$
\left(u_{\alpha_{k}}, v_{k}\right)_{H_{\alpha_{k}}}=\frac{1}{k}\left\|u_{\alpha_{k}}\right\|_{H_{\alpha_{k}}} \geq 1
$$

This is a contradiction and thus we obtain $\sup _{\alpha}\left\|u_{\alpha}\right\|<\infty$.

Let $\left\{v_{\alpha}\right\}$ be a net with $v_{\alpha} \in H_{\alpha}$ which strongly converges to $u$. Then, $\left(u_{\alpha}, v_{\alpha}\right)_{H_{\alpha}} \rightarrow(u, u)_{H}$. Hence,

$$
\begin{aligned}
0 & \leq \underline{\lim }\left\|u_{\alpha}-v_{\alpha}\right\|_{H_{\alpha}}^{2} \\
& =\underline{\lim }\left(\left\|u_{\alpha}\right\|_{H_{\alpha}}^{2}-2 \operatorname{Re}\left(u_{\alpha}, v_{\alpha}\right)_{H_{\alpha}}+\left\|v_{\alpha}\right\|_{H_{\alpha}}^{2}\right) \\
& =\underline{\lim }\left\|u_{\alpha}\right\|_{H_{\alpha}}^{2}-\|u\|_{H}^{2} .
\end{aligned}
$$

This completes the proof of the first assertion. The second also follows from the above and Lemma 2.1(5),(6).

Lemma 2.4. Let $u \in H$ and let $\left\{u_{\alpha}\right\}_{\alpha \in \mathcal{A}}$ be a net of vectors $u_{\alpha} \in H_{\alpha}$. Then, $u_{\alpha} \rightarrow u$ strongly if and only if $\left(u_{\alpha}, v_{\alpha}\right)_{H_{\alpha}} \rightarrow(u, v)_{H}$ for any net $\left\{v_{\alpha}\right\}_{\alpha \in \mathcal{A}}$ of vectors $v_{\alpha} \in H_{\alpha}$ weakly tending to a vector $v \in H$.

Proof. The 'only if' part is trivial. We prove the 'if' part. The assumption implies that $u_{\alpha} \rightarrow u$ weakly. Setting $v_{\alpha}:=u_{\alpha}$ and $v:=u$ in the assumption, we have $\left\|u_{\alpha}\right\|_{H_{\alpha}} \rightarrow\|u\|_{H}$. This completes the proof.

\subsection{Convergence of bounded operators.}

Denote by $L(H)$ the set of bounded linear operators on $H$, and by $\|\cdot\|_{L(H)}$ the operator norm. Let $B \in L(H)$ and $B_{\alpha} \in L\left(H_{\alpha}\right)$ for all $\alpha \in \mathcal{A}$.

Definition 2.6 (Convergence of operators). We say that $\left\{B_{\alpha}\right\}_{\alpha \in \mathcal{A}}$ strongly (resp. weakly) converges to $B$ if $B_{\alpha} u_{\alpha} \rightarrow B u$ strongly (resp. weakly) for any net $\left\{u_{\alpha}\right\}_{\alpha \in \mathcal{A}}$ with $u_{\alpha} \in H_{\alpha}$ strongly (resp. weakly) tending to a $u \in H$. We say that $\left\{B_{\alpha}\right\}_{\alpha \in \mathcal{A}}$ compactly converges to $B$ if $B_{\alpha} u_{\alpha} \rightarrow B u$ weakly for any net $\left\{u_{\alpha}\right\}_{\alpha \in \mathcal{A}}$ with $u_{\alpha} \in H_{\alpha}$ strongly tending to a $u \in H$. The topology induced from the strong convergence is called the strong topology on $L(\mathcal{H}):=\bigsqcup_{\nu \in \mathcal{N}} L\left(H_{\nu}\right)$.

Remark that if $H_{\alpha}=H$ (i.e., $\nu_{\alpha}=\nu$ ) for all $\alpha \in \mathcal{A}$, the concept of the strong and weak convergence in $L(\mathcal{H})$ defined here is different from the 
ordinary one (cf. Lemma 2.8). The strong topology on $L(\mathcal{H})$ is Hausdorff if so is $\left\{H_{\nu}\right\}_{\nu \in \mathcal{N}}$. It is clear that if $B_{\alpha} \rightarrow B$ compactly, then $B_{\alpha} \rightarrow B$ strongly and weakly. The compact convergence does not induce a topology on $L(\mathcal{H})$ as is seen in Remark 2.3 below.

Lemma 2.5. Let $u, v \in H$ be any vectors and $\left\{u_{\alpha}\right\}_{\alpha \in \mathcal{A}},\left\{v_{\alpha}\right\}_{\alpha \in \mathcal{A}}$ any nets of vectors with $u_{\alpha}, v_{\alpha} \in H_{\alpha}$. Then we have the following:

(1) $B_{\alpha} \rightarrow B$ strongly if and only if

$$
\lim _{\alpha}\left(B_{\alpha} u_{\alpha}, v_{\alpha}\right)_{H_{\alpha}}=(B u, v)_{H}
$$

for any $\left\{u_{\alpha}\right\},\left\{v_{\alpha}\right\}, u, v$ such that $u_{\alpha} \rightarrow u$ strongly and $v_{\alpha} \rightarrow v$ weakly.

(2) $B_{\alpha} \rightarrow B$ weakly if and only if (2.6) holds for any $\left\{u_{\alpha}\right\},\left\{v_{\alpha}\right\}, u, v$ such that $u_{\alpha} \rightarrow u$ weakly and $v_{\alpha} \rightarrow v$ strongly.

(3) $B_{\alpha} \rightarrow B$ compactly if and only if (2.6) holds for any $\left\{u_{\alpha}\right\},\left\{v_{\alpha}\right\}, u, v$ such that $u_{\alpha} \rightarrow u$ weakly and $v_{\alpha} \rightarrow v$ weakly.

Proof. The lemma follows from the definitions of convergences and Lemma 2.4 .

Denote by $A^{*}$ the adjoint of an operator $A$. The following is a direct consequence of Lemma 2.5 .

Lemma 2.6. (1) $B_{\alpha} \rightarrow B$ strongly if and only if $B_{\alpha}^{*} \rightarrow B^{*}$ weakly. In particular, the strong convergence is equivalent to the weak convergence for symmetric operators.

(2) $B_{\alpha} \rightarrow B$ compactly if and only if $B_{\alpha}^{*} \rightarrow B^{*}$ compactly.

Lemma 2.7. If $B_{\alpha} \rightarrow B$ compactly, then $B$ and $B^{*}$ are both compact operators.

Proof. Note that the compactness of $B$ is equivalent to that of $B^{*}$. Let $\left\{u_{\beta}\right\}_{\beta \in \mathcal{B}}$ be a net of vectors in $H$ weakly converging to a vector $u \in H$. It suffices to prove that $B^{*} u_{\beta} \rightarrow B^{*} u H$-strongly. We easily see that $B^{*} u_{\beta} \rightarrow$ $B^{*} u H$-weakly. For each $\beta \in \mathcal{B}$, there is a net $\left\{u_{\beta, \alpha}\right\}_{\alpha \in \mathcal{A}}$ with $u_{\beta, \alpha} \in H_{\alpha}$ such that $\lim _{\alpha} u_{\beta, \alpha}=u_{\beta} \mathcal{H}$-strongly. Since $B_{\alpha}^{*} \rightarrow B^{*}$ strongly, we have 
$\lim _{\alpha} B_{\alpha}^{*} u_{\beta, \alpha}=B^{*} u_{\beta} \mathcal{H}$-strongly. A diagonal argument yields that there is a subnet $\{\alpha(\beta)\}_{\beta \in \mathcal{B}}$ of $\mathcal{A}$ such that

$$
\begin{gathered}
\lim _{\beta} u_{\beta, \alpha(\beta)}=u \quad \mathcal{H} \text {-weakly, } \\
\lim _{\beta}\left|\left\|B_{\alpha(\beta)}^{*} u_{\beta, \alpha(\beta)}\right\|_{H_{\alpha(\beta)}}-\left\|B^{*} u_{\beta}\right\|_{H}\right|=0 .
\end{gathered}
$$

The compact convergence $B_{\alpha}^{*} \rightarrow B^{*}$ and (2.7) together show that $\lim _{\beta} B_{\alpha(\beta)}^{*} u_{\beta, \alpha(\beta)}=B^{*} u \mathcal{H}$-strongly. Hence, by (2.8), $\left\|B^{*} u_{\beta}\right\|_{H} \rightarrow\left\|B^{*} u\right\|_{H}$ and so $B^{*} u_{\beta} \rightarrow B^{*} u H$-strongly. This completes the proof.

Remark 2.3. If $B$ is a noncompact operator, then $B_{\alpha}:=B$ does not compactly converge to $B$ by Lemma 2.7 . Therefore, the compact convergence on $L(\mathcal{H})$ does not induce a topology on $L(\mathcal{H})$ in general and induces a topology only on the set of compact operators in $L(\mathcal{H})$.

Lemma 2.8. Assume that $H_{\alpha}=H$ (i.e., $\nu_{\alpha}=\nu$ ) for all $\alpha \in \mathcal{A}$. Then we have the following (1) and (2).

(1) Supposing $\left\{\left\|B_{\alpha}\right\|_{L(H)}\right\}$ is uniformly bounded, we obtain $B_{\alpha} \rightarrow B$ strongly in $L(\mathcal{H})$ if and only if $B_{\alpha} \rightarrow B$ strongly in $L(H)$, i.e., $\lim _{\alpha}\left\|B_{\alpha} u-B u\right\|_{H}=0$ for any $u \in H$.

(2) $B_{\alpha} \rightarrow B$ compactly in $L(\mathcal{H})$ if and only if $\left\|B_{\alpha}-B\right\|_{L(H)} \rightarrow 0$ and $B$ is compact.

Proof. (1) follows from

$$
\left\|B_{\alpha} u_{\alpha}-B_{\alpha} u\right\|_{H_{\alpha}} \leq\left\|B_{\alpha}\right\|_{L(H)}\left\|u_{\alpha}-u\right\|_{H} .
$$

(2): Assume that $B_{\alpha} \rightarrow B$ compactly in $L(\mathcal{H})$. The compactness of $B$ follows from Lemma 2.7. To prove $\left\|B_{\alpha}-B\right\|_{L(H)} \rightarrow 0$, we suppose that $\underline{\lim }_{\alpha}\left\|B_{\alpha}-B\right\|_{L(H)}>0$. There exists a net of unit vectors $u_{\alpha} \in H$ such that

$$
\lim _{\alpha}\left|\left\|B_{\alpha}-B\right\|_{L(H)}-\left\|B_{\alpha} u_{\alpha}-B u_{\alpha}\right\|_{H}\right|=0 .
$$

By replacing a subnet, $u_{\alpha}$ weakly converges to some vector $u \in H$ with $\|u\|_{H} \leq 1$, which together with the compact convergence $B_{\alpha} \rightarrow B$ implies $B_{\alpha} u_{\alpha} \rightarrow B u$ strongly. Besides, by the compactness of $B$, we have $B u_{\alpha} \rightarrow$ $B u$ strongly. Thus, by (2.9), we obtain $\left\|B_{\alpha}-B\right\|_{L(H)} \rightarrow 0$, which is a contradiction. 
Conversely, we assume that $\left\|B_{\alpha}-B\right\|_{L(H)} \rightarrow 0$ and $B$ is compact. For any net $u_{\alpha} \in H$ weakly converging to a vector $u \in H$, we have

$$
\left\|B_{\alpha} u_{\alpha}-B u\right\|_{H} \leq\left\|B_{\alpha}-B\right\|_{L(H)}\left\|u_{\alpha}\right\|_{H}+\left\|B u_{\alpha}-B u\right\|_{H} \rightarrow 0 .
$$

This completes the proof.

Lemma 2.9. (1) If $B_{\alpha} \rightarrow B$ strongly, then

$$
\varliminf_{\alpha}\left\|B_{\alpha}\right\|_{L\left(H_{\alpha}\right)} \geq\|B\|_{L(H)} .
$$

(2) If $B_{\alpha} \rightarrow B$ compactly, then

$$
\lim _{\alpha}\left\|B_{\alpha}\right\|_{L\left(H_{\alpha}\right)}=\|B\|_{L(H)} .
$$

Proof. (1): For any $\epsilon>0$ there is a unit vector $u \in H$ such that $\|B u\|_{H}>$ $\|B\|_{L(H)}-\epsilon$. Find a net $u_{\alpha} \in H_{\alpha}$ strongly converging to $u$. Note that $\left\|u_{\alpha}\right\|_{H_{\alpha}} \rightarrow 1$. Since $B_{\alpha} \rightarrow B$ strongly, we have $\left\|B_{\alpha} u_{\alpha}\right\|_{H_{\alpha}} \rightarrow\|B u\|_{H}$ and therefore,

$$
\underline{\lim }\left\|B_{\alpha}\right\|_{L\left(H_{\alpha}\right)} \geq \underline{\lim } \frac{\left\|B_{\alpha} u_{\alpha}\right\|_{H_{\alpha}}}{\left\|u_{\alpha}\right\|_{H_{\alpha}}}=\|B u\|_{H}>\|B\|_{L(H)}-\epsilon .
$$

This completes the proof of (1).

(2): There is a net of unit vectors $u_{\alpha} \in H_{\alpha}$ such that $\mid\left\|B_{\alpha}\right\|_{L\left(H_{\alpha}\right)}-$ $\left\|B_{\alpha} u_{\alpha}\right\| \mid \rightarrow 0$. Replacing with a subnet of $\mathcal{A}$, we assume that $u_{\alpha}$ weakly converges a vector $u \in H$ with $\|u\|_{H} \leq 1$. Since $B_{\alpha} u_{\alpha} \rightarrow B u$ strongly by the assumption, we have

$$
\|B\|_{L(H)} \geq \frac{\|B u\|_{H}}{\|u\|_{H}} \geq\|B u\|_{H}=\lim _{\alpha}\left\|B_{\alpha} u_{\alpha}\right\|_{H_{\alpha}}=\lim _{\alpha}\left\|B_{\alpha}\right\|_{L\left(H_{\alpha}\right)} .
$$

This together with (1) completes the proof.

\subsection{Convergence of spectral measures on complex Hilbert spaces.}

Throughout this section, we assume that $H$ and $H_{\alpha}$ have complex coefficient $K=\mathbb{C}$ under Assumption 2.1. Let $A$ and $A_{\alpha}$ be selfadjoint operators on $H$ and $H_{\alpha}$ respectively. Denote by $E$ and $E_{\alpha}$ the spectral measures of $A$ and $A_{\alpha}$, and by $\left\{R_{\zeta}\right\}_{\zeta \in \rho(A)}$ and $\left\{R_{\zeta}^{\alpha}\right\}_{\zeta \in \rho\left(A_{\alpha}\right)}$ their resolvents (i.e., $R_{\zeta}:=$ $(\zeta-A)^{-1}$ and $\left.R_{\zeta}^{\alpha}:=\left(\zeta-A_{\alpha}\right)^{-1}\right)$, where $\rho(\cdot)$ denotes the resolvent set. We 
say that a continuous function $\varphi: \mathbb{R} \rightarrow \mathbb{C}$ vanishes at infinity if and only if $\lim _{|x| \rightarrow \infty} f(x)=0$. We set $i:=\sqrt{-1}$ and indicate by $\operatorname{Im} \zeta$ the imaginary part of a $\zeta \in \mathbb{C}$.

Theorem 2.1. The following are all equivalent:

(1) $R_{\zeta}^{\alpha} \rightarrow R_{\zeta}$ strongly (resp. compactly) for some $\zeta \in \mathbb{C}$ with $\operatorname{Im} \zeta \neq 0$.

(2) $\varphi\left(A_{\alpha}\right) \rightarrow \varphi(A)$ strongly (resp. compactly) for any continuous function $\varphi: \mathbb{R} \rightarrow \mathbb{C}$ with compact support.

(3) $\varphi_{\alpha}\left(A_{\alpha}\right) \rightarrow \varphi(A)$ strongly (resp. compactly) for any net $\left\{\varphi_{\alpha}: \mathbb{R} \rightarrow \mathbb{C}\right\}$ of continuous functions vanishing at infinity which uniformly converges to a continuous function $\varphi: \mathbb{R} \rightarrow \mathbb{C}$ vanishing at infinity.

(4) $E_{\alpha}((\lambda, \mu]) \rightarrow E((\lambda, \mu])$ strongly (resp. compactly) for any two real numbers $\lambda<\mu$ which are not in the point spectrum of $A$.

(5) $\left(E_{\alpha} u_{\alpha}, v_{\alpha}\right)_{H_{\alpha}} \rightarrow(E u, v)_{H}$ vaguely for any nets $\left\{u_{\alpha}\right\}_{\alpha \in \mathcal{A}},\left\{v_{\alpha}\right\}_{\alpha \in \mathcal{A}}$ of vectors $u_{\alpha}, v_{\alpha} \in H_{\alpha}$ and any $u, v \in H$ such that $u_{\alpha} \rightarrow u$ strongly and $v_{\alpha} \rightarrow v$ weakly (resp. $u_{\alpha} \rightarrow u$ weakly and $v_{\alpha} \rightarrow v$ weakly).

Proof. It is trivial that (3) implies (1) and (2).

Let us prove $(1) \Longrightarrow(2)$. The idea of this proof is essentially due to XI $\S 11.4$ of [48]. Consider the set $\mathbf{A}$ of continuous functions $\varphi: \mathbb{R} \rightarrow \mathbb{C}$ vanishing at infinity such that $\varphi\left(A_{\alpha}\right) \rightarrow \varphi(A)$ strongly (resp. compactly). Note that for any bounded continuous functions $\varphi, \psi: \mathbb{R} \rightarrow \mathbb{C}$, we have

$$
\left\|\varphi\left(A_{\alpha}\right)-\psi\left(A_{\alpha}\right)\right\|_{L\left(H_{\alpha}\right)},\|\varphi(A)-\psi(A)\|_{L(H)} \leq \sup _{x \in \mathbb{R}}|\varphi(x)-\psi(x)|,
$$

which proves that a uniform limit of functions in $\mathbf{A}$ is also a function in A. Thus, A is a uniformly closed algebra which is closed under complex conjugation. By (1), it contains the function $x \mapsto(\zeta-x)^{-1}$ and so separates the points on $\mathbb{R}$ (i.e., $\left.x \neq y \Longrightarrow(\zeta-x)^{-1} \neq(\zeta-y)^{-1}\right)$. Thus, by the Stone-Weierstrass theorem, $\mathbf{A}$ contains all continuous functions vanishing at infinity, which implies (2).

Let $u, v \in H$ and let $\left\{u_{\alpha}\right\}_{\alpha \in \mathcal{A}},\left\{v_{\alpha}\right\}_{\alpha \in \mathcal{A}}$ be two nets of vectors $u_{\alpha}, v_{\alpha} \in$ $H_{\alpha}$ such that $u_{\alpha} \rightarrow u$ strongly and $v_{\alpha} \rightarrow v$ weakly (resp. $u_{\alpha} \rightarrow u$ weakly and $v_{\alpha} \rightarrow v$ weakly). Letting $a_{\alpha}:=\left(E_{\alpha} u_{\alpha}, v_{\alpha}\right)_{H_{\alpha}}$ and $a:=(E u, v)_{H}$, we have

$$
(\varphi(A) u, v)_{H}=\int_{\mathbb{R}} \varphi d a, \quad a((\lambda, \mu])=((E(\lambda, \mu]) u, v)_{H},
$$


and also the similar formulas for $a_{\alpha}, A_{\alpha}, E_{\alpha}((\lambda, \mu])$. Thus, by Lemma 2.5, we obtain the equivalence between $(2)-(5)$.

Remark 2.4. If one (or all) of the compact convergence conditions of Theorem 2.1 holds, then, by Lemma 2.7 , all $R_{\zeta}$ and $E((\lambda, \mu])$ are compact operators, and in particular $A$ has only discrete spectrum.

Definition 2.7. The strong graph limit $\Gamma_{\infty}$ of $\left\{A_{\alpha}\right\}_{\alpha \in \mathcal{A}}$ is defined to be the set of pairs $(u, v) \in H \times H$ such that there exists a net of vectors $u_{\alpha} \in \mathcal{D}\left(A_{\alpha}\right)$ with $u_{\alpha} \rightarrow u$ and $A_{\alpha} u_{\alpha} \rightarrow v$ strongly.

Theorem 2.2. Only for the strong convergences, the conditions (1)-(5) of Theorem 2.1 are also equivalent to each of the following:

(6) $e^{i t A_{\alpha}} \rightarrow e^{i t A}$ strongly for any $t \in \mathbb{R}$.

(7) $\varphi_{\alpha}\left(A_{\alpha}\right) \rightarrow \varphi(A)$ strongly for any net $\left\{\varphi_{\alpha}: \mathbb{R} \rightarrow \mathbb{C}\right\}$ of bounded continuous functions uniformly converging to a bounded continuous function $\varphi: \mathbb{R} \rightarrow \mathbb{C}$.

(8) The strong graph limit $\Gamma_{\infty}$ of $\left\{A_{\alpha}\right\}_{\alpha \in \mathcal{A}}$ coincides with the graph of $A$.

Proof. It is trivial that $(7) \Longrightarrow(3),(6)$.

Let us prove $(6) \Longrightarrow(2)$. Let $H_{\alpha} \ni u_{\alpha} \rightarrow u \in H$ strongly and $H_{\alpha} \ni v_{\alpha} \rightarrow v \in H$ weakly. Then, by (6),

$$
\left(e^{i t A_{\alpha}} u_{\alpha}, v_{\alpha}\right)_{H_{\alpha}} \rightarrow\left(e^{i t A} u, v\right)_{H}
$$

for any $t \in \mathbb{R}$. The dominated convergence theorem shows that for any $\varphi \in L^{1}(\mathbb{R})$,

$$
\int_{-\infty}^{\infty}\left(\varphi(t) e^{i t A_{\alpha}} u_{\alpha}, v_{\alpha}\right)_{H_{\alpha}} d t \rightarrow \int_{-\infty}^{\infty}\left(\varphi(t) e^{i t A} u, v\right)_{H} d t
$$

and hence $\left(\hat{\varphi}\left(A_{\alpha}\right) u_{\alpha}, v_{\alpha}\right)_{H_{\alpha}} \rightarrow(\hat{\varphi}(A) u, v)_{H}$, where $\hat{\varphi}$ denotes the Fourier transform of $\varphi$. Thus, $\hat{\varphi}\left(A_{\alpha}\right) \rightarrow \hat{\varphi}(A)$ strongly. Since $\left\{\hat{\varphi} \mid \varphi \in L^{1}(\mathbb{R})\right\}$ is dense in the set of continuous functions with compact support with respect to the uniform norm, we obtain (2).

Let us prove (4),(5) $\Longrightarrow(7)$. Let $H_{\alpha} \ni u_{\alpha} \rightarrow u \in H$ strongly, $H_{\alpha} \ni$ $v_{\alpha} \rightarrow v \in H$ weakly, $a_{\alpha}:=\left(E_{\alpha} u_{\alpha}, v_{\alpha}\right)_{H_{\alpha}}$, and $a:=(E u, v)_{H}$. We take any two real numbers $\lambda<\mu$ which are not point spectrum of $A$, and a continuous function $h: \mathbb{R} \rightarrow[0,1]$ with compact support such that $h=1$ on 
the interval $I:=(\lambda, \mu]$. We note that the identity operator on $H_{\alpha}$ strongly converges to that on $H$, which implies $a_{\alpha}(\mathbb{R}) \rightarrow a(\mathbb{R})$. Thus, (4) shows that $a_{\alpha}(I), a_{\alpha}(\mathbb{R} \backslash I)$ respectively converges to $a(I), a(\mathbb{R} \backslash I)$. Since $a_{\alpha} \rightarrow a$ vaguely and $h \varphi_{\alpha} \rightarrow h \varphi$ uniformly, we obtain

$$
\begin{aligned}
& \varlimsup_{\alpha}\left|\int_{\mathbb{R}} \varphi_{\alpha} d a_{\alpha}-\int_{\mathbb{R}} \varphi d a\right| \\
& \leq \varlimsup_{\alpha}\left|\int_{\mathbb{R}} h \varphi_{\alpha} d a_{\alpha}-\int_{\mathbb{R}} h \varphi d a\right| \\
& \quad+\varlimsup_{\alpha} \int_{\mathbb{R}}(1-h)\left|\varphi_{\alpha}\right| d a_{\alpha}+\int_{\mathbb{R}}(1-h)|\varphi| d a \\
& \leq 2 \sup |\varphi| a(\mathbb{R} \backslash I) .
\end{aligned}
$$

The arbitrariness of $I$ leads to the convergence

$$
\left(\varphi_{\alpha}\left(A_{\alpha}\right) u_{\alpha}, v_{\alpha}\right)_{H_{\alpha}}=\int_{\mathbb{R}} \varphi_{\alpha} d a_{\alpha} \rightarrow \int_{\mathbb{R}} \varphi d a=(\varphi(A) u, v)_{H},
$$

which shows (7).

To prove $(1) \Longrightarrow(8)$, we assume $R_{i}^{\alpha} \rightarrow R_{i}$ strongly. For any $u \in \mathcal{D}(A)$, there exists a net of vectors $w_{\alpha} \in H_{\alpha}$ strongly converging to $(i-A) u$. Setting $u_{\alpha}:=R_{i}^{\alpha} w_{\alpha}$, we have $u_{\alpha} \rightarrow R_{i}(i-A) u=u$ by the assumption. Moreover,

$$
A_{\alpha} u_{\alpha}=i u_{\alpha}-w_{\alpha} \rightarrow i u-(i-A) u=A u .
$$

This implies $(u, A u) \in \Gamma_{\infty}$. Thus, the graph of $A$ is contained in $\Gamma_{\infty}$.

Conversely, we assume that $\mathcal{D}\left(A_{\alpha}\right) \ni u_{\alpha} \rightarrow u \in H$ and $A_{\alpha} u_{\alpha} \rightarrow v \in H$. Since $\left(i-A_{\alpha}\right) u_{\alpha} \rightarrow i u-v$, the assumption shows $u_{\alpha}=R_{i}^{\alpha}\left(i-A_{\alpha}\right) u_{\alpha} \rightarrow$ $R_{i}(i u-v)=: w$. Here we must have $u=w$. It follows that

$$
A u=A w=i R_{i}(i u-v)+v-i u=v .
$$

Thus we obtain (8).

We will at last prove $(8) \Longrightarrow(1)$. By $(8)$, for any $w \in \mathcal{D}(A)$ there exists a net of vectors $w_{\alpha} \in \mathcal{D}\left(A_{\alpha}\right)$ with $w_{\alpha} \rightarrow w$ and $A_{\alpha} w_{\alpha} \rightarrow A w$. It follows that

$$
\begin{gathered}
\left(i-A_{\alpha}\right) w_{\alpha} \rightarrow(i-A) w \\
R_{i}^{\alpha}\left(i-A_{\alpha}\right) w_{\alpha}=w_{\alpha} \rightarrow w=R_{i}(i-A) w .
\end{gathered}
$$

Since $(i-A) H=H$, i.e., $(i-A) w, w \in \mathcal{D}(A)$, runs over all vectors in $H$, the above implies that for any $u \in H$ there exists a net of vectors $u_{\alpha} \in H$ 
with $u_{\alpha} \rightarrow u$ and $R_{i}^{\alpha} u_{\alpha} \rightarrow R_{i} u$. Taking any net of vectors $v_{\alpha} \in H_{\alpha}$ with $v_{\alpha} \rightarrow u$, we have

$$
\left\|R_{i}^{\alpha} u_{\alpha}-R_{i}^{\alpha} v_{\alpha}\right\|_{H_{\alpha}} \leq\left\|u_{\alpha}-v_{\alpha}\right\|_{H_{\alpha}} \rightarrow 0
$$

and therefore $R_{i}^{\alpha} v_{\alpha} \rightarrow R_{i} u$. This completes the proof of the theorem.

Remark 2.5. The identity operator on $H_{\alpha}$ does not compactly converge to that on $H$ provided $H$ has infinite dimensional. In particular, Theorem 2.2(7) does not hold for the compact convergence in general.

\subsection{Convergence of quadratic forms.}

First we introduce the extended notion of $\Gamma$-convergence of functions on $H_{\alpha}$ with values in $\overline{\mathbb{R}}:=\mathbb{R} \cup\{-\infty, \infty\}$. Compare [18].

Definition 2.8 ( $\Gamma$-convergence). Under Assumption 2.1, we say that a net $\left\{F_{\alpha}: H_{\alpha} \rightarrow \overline{\mathbb{R}}\right\}_{\alpha \in \mathcal{A}}$ of functions $\Gamma$-converges to a function $F: H \rightarrow \overline{\mathbb{R}}$ (or $F$ is a $\Gamma$-limit of $\left\{F_{\alpha}\right\}_{\alpha \in \mathcal{A}}$ ) if and only if the two following (F1) and (F2) hold:

(F1) If a net $\left\{u_{\alpha}\right\}_{\alpha \in \mathcal{A}}$ with $u_{\alpha} \in H_{\alpha}$ strongly converges to a $u \in H$ in $\mathcal{H}$, then

$$
F(u) \leq \frac{\lim }{\alpha} F_{\alpha}\left(u_{\alpha}\right)
$$

(F2) For any $u \in H$ there exists a net $\left\{u_{\alpha}\right\}_{\alpha \in \mathcal{A}}$ with $u_{\alpha} \in H_{\alpha}$ which strongly converges to $u$ in $\mathcal{H}$ and

$$
F(u)=\lim _{\alpha} F_{\alpha}\left(u_{\alpha}\right)
$$

Lemma 2.10. If a net $\left\{F_{\alpha}: H_{\alpha} \rightarrow \overline{\mathbb{R}}\right\}_{\alpha \in \mathcal{A}}$ of functions $\Gamma$-converges to a function $F: H \rightarrow \overline{\mathbb{R}}$, then $F$ is lower semi-continuous.

Proof. Let $\left\{u_{\beta}\right\}_{\beta \in \mathcal{B}}$ be a net of vectors in $H$ strongly converging to a vector $u \in H$. Then, by (F2), for each $\beta \in \mathcal{B}$ there exists a net $\left\{u_{\beta}^{\alpha}\right\}_{\alpha \in \mathcal{A}}$ with $u_{\beta}^{\alpha} \in H_{\alpha}$ such that $\lim _{\alpha} u_{\beta}^{\alpha}=u_{\beta}$ (strongly) and $\lim _{\alpha} F_{\alpha}\left(u_{\beta}^{\alpha}\right)=F\left(u_{\beta}\right)$. For a net $\epsilon_{\beta} \searrow 0$, we find a subnet $\left\{\alpha_{\beta}\right\}_{\beta \in \mathcal{B}}$ of $\mathcal{A}$ such that $u_{\beta}^{\alpha_{\beta}} \rightarrow u$ strongly and

$$
\left|F\left(u_{\beta}\right)-F_{\alpha_{\beta}}\left(u_{\beta}^{\alpha_{\beta}}\right)\right|<\epsilon_{\beta} \quad \text { for any } \beta \in \mathcal{B} \text {. }
$$


Therefore, by (F1),

$$
\underline{\varliminf_{\beta}} F\left(u_{\beta}\right)=\varliminf_{\beta} F_{\alpha_{\beta}}\left(u_{\beta}^{\alpha_{\beta}}\right) \geq F(u) .
$$

This completes the proof.

A quadratic form on a Hilbert space $H$ over $K$ is, by definition, a sesquilinear (or bilinear if $K=\mathbb{R}$ ) form $\mathcal{E}: \mathcal{D}(\mathcal{E}) \times \mathcal{D}(\mathcal{E}) \rightarrow K$, where $\mathcal{D}(\mathcal{E}) \subset H$ is a (not necessarily dense) linear subspace. We assume that any quadratic form $\mathcal{E}$ in this paper is nonnegative and symmetric, i.e., $u \mapsto \mathcal{E}(u, v)$ is linear, $\mathcal{E}(u, v)=\overline{\mathcal{E}(v, u)}$, and $\mathcal{E}(u, u) \geq 0$ for any $u, v \in \mathcal{D}(\mathcal{E})$. Note that $\mathcal{E}_{1}(u, v):=(u, v)_{H}+\mathcal{E}(u, v), u, v \in \mathcal{D}(\mathcal{E})$, is also a (nonnegative and symmetric) quadratic form, so that $\mathcal{D}(\mathcal{E})$ with $\mathcal{E}_{1}$ becomes a (not necessarily complete) inner product space over $K$. We say that $\mathcal{E}$ is closed if and only if $\mathcal{D}(\mathcal{E})$ is $\mathcal{E}_{1}$-complete. We sometimes identify a quadratic form $\mathcal{E}$ on $H$ with a function $H \ni u \mapsto \mathcal{E}(u, u)=: \mathcal{E}(u) \in \overline{\mathbb{R}}$ by setting $\mathcal{E}(u):=\infty$ for $u \in H \backslash \mathcal{D}(\mathcal{E})$. Then, the closedness of $\mathcal{E}$ is equivalent to the lower semi-continuity of $\mathcal{E}: H \rightarrow \overline{\mathbb{R}}$.

Lemma 2.11. If a net $\left\{\mathcal{E}_{\alpha}\right\}_{\alpha \in \mathcal{A}}$ of quadratic forms $\mathcal{E}_{\alpha}$ on $H_{\alpha} \Gamma$-converges to a function $F: H \rightarrow \overline{\mathbb{R}}$, then $F$ is identified with a closed quadratic form on $H$.

Proof. To see that $F$ is identified with a quadratic form, it suffices to show that for any $u, v \in H$ and $a \in K$,

$$
\begin{gathered}
F(u) \geq 0, \quad F(a u)=|a|^{2} F(u), \\
F(u+v)+F(u-v)=2(F(u)+F(v)) .
\end{gathered}
$$

In fact, these are proved by an easy discussion using (F2). The closedness of $F$ follows from Lemma 2.10.

Denote by $\mathcal{F}(\mathcal{H})$ the set of closed quadratic forms on $H_{\nu}, \nu \in \mathcal{N}$.

Proposition 2.3. The $\Gamma$-convergence induces a topology on $\mathcal{F}(\mathcal{H})$. It is Hausdorff if $\left\{H_{\nu}\right\}_{\nu \in \mathcal{N}}$ is Hausdorff.

Proof. If $H_{\alpha}=H$ and $\mathcal{E}_{\alpha}=\mathcal{E}$ for all $\alpha \in \mathcal{A}$, then $\mathcal{E}_{\alpha} \Gamma$-converges to $\mathcal{E}$ provided $\mathcal{E}$ is a closed quadratic form. This together with (F1) and (F2) 
shows the first assertion. If $\left\{H_{\nu}\right\}_{\nu \in \mathcal{N}}$ is Hausdorff, then (F2) implies the uniqueness of a $\Gamma$-limit.

Definition 2.9 ( $\Gamma$-topology). We call the topology induced from the $\Gamma$ convergence on $\mathcal{F}(\mathcal{H})$ the $\Gamma$-topology.

Our next purpose is to prove:

Theorem 2.3. Any net $\left\{\mathcal{E}_{\alpha}\right\}_{\alpha \in \mathcal{A}}$ of quadratic forms $\mathcal{E}_{\alpha}$ on $H_{\alpha}$ has a $\Gamma$ convergent subnet whose $\Gamma$-limit is a closed quadratic form on $H$. In particular, $\mathcal{F}(\mathcal{H})$ is sequentially compact with respect to the $\Gamma$-topology provided $\left\{H_{\nu}\right\}_{\nu \in \mathcal{N}}$ is sequentially compact.

In order to prove this theorem, we need to recall the definition of $\Gamma$-limit of functions on a topological space $X$, due to E. De Giorgi. Note that if we assume the first countability of $X$, the following definition is equivalent to the original one.

Definition 2.10 ( $\Gamma$-convergence (original)). Let $X$ be a topological space. We say that a net $\left\{F_{\beta}: X \rightarrow \overline{\mathbb{R}}\right\}_{\beta \in \mathcal{B}}$ of functions $\Gamma$-converges to a function $F: X \rightarrow \overline{\mathbb{R}}$ (or $F$ is a $\Gamma$-limit of $\left\{F_{\alpha}\right\}_{\alpha \in \mathcal{A}}$ ) if and only if the two following (G1) and (G2) hold:

(G1) If a net $\left\{x_{\beta}\right\}_{\beta \in \mathcal{B}}$ of points in $X$ converges to a point $x \in X$, then

$$
F(x) \leq \varliminf_{\beta} F_{\beta}\left(x_{\beta}\right) .
$$

(G2) For any $x \in X$ there exists a net $\left\{x_{\beta}\right\}_{\beta \in \mathcal{B}}$ of points in $X$ converging to $x$ such that

$$
F(x)=\lim _{\beta} F_{\beta}\left(x_{\beta}\right) .
$$

We apply the above definition to $X:=\mathcal{H}=\bigsqcup_{\nu \in \mathcal{N}} H_{\nu}$ with the strong topology. For a function $F: \mathcal{H} \rightarrow \overline{\mathbb{R}}$ we set $\mathcal{D}(F):=\{u \in \mathcal{H} \mid F(u)<\infty\}$. The following describes the connection between Definitions 2.8 and 2.10.

Lemma 2.12. Let $\left\{H_{\beta}\right\}_{\beta \in \mathcal{B}}$ be a net of $\left\{H_{\nu}\right\}_{\nu \in \mathcal{N}}$ and $\left\{F_{\beta}: \mathcal{H} \rightarrow \overline{\mathbb{R}}\right\}_{\beta \in \mathcal{B}}$ a $\Gamma$-convergent (in the sense of Definition 2.10) net of functions such that $\mathcal{D}\left(F_{\beta}\right) \subset H_{\beta}$ for each $\beta \in \mathcal{B}$. Then, the $\Gamma$-limit $F: \mathcal{H} \rightarrow \overline{\mathbb{R}}$ of $\left\{F_{\beta}\right\}$ satisfies

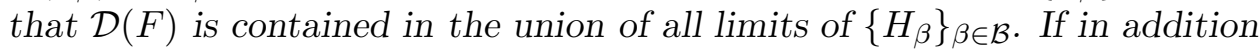
$H_{\beta}$ converges to an $H_{\infty} \in\left\{H_{\nu}\right\}_{\nu \in \mathcal{N}}$, then $\left.F_{\beta}\right|_{H_{\beta}} \Gamma$-converges to $\left.F\right|_{H_{\infty}}$ in the sense of Definition 2.8 . 
Proof. Let $H \in\left\{H_{\nu}\right\}_{\nu \in \mathcal{N}}$ be any Hilbert space which is not a limit of $\left\{H_{\beta}\right\}_{\beta \in \mathcal{B}}$. Then, there exists a neighborhood $\mathcal{U}$ at $H$ in $\left\{H_{\nu}\right\}_{\nu \in \mathcal{N}}$ such that $H_{\beta} \notin \mathcal{U}$ for all sufficiently large $\beta \in \mathcal{B}$. It then follows that for sufficiently large $\beta \in \mathcal{B}, F_{\beta}=\infty$ on each $H^{\prime} \in \mathcal{U}$. Since $\bigsqcup \mathcal{U} \subset \mathcal{H}$ is a neighborhood at each $u \in H$ in $\mathcal{H}$ and by (G2), we have $F=\infty$ on $H$, i.e., $\mathcal{D}(F) \cap H=\emptyset$. This completes the proof of the first assertion. The second is clear.

We know the following:

Fact 2.1 (Theorem 8.5 of [18]). If $X$ is a second countable topological space, then any net $\left\{F_{\beta}: X \rightarrow \overline{\mathbb{R}}\right\}_{\beta \in \mathcal{B}}$ of functions has a $\Gamma$-convergent subnet.

We intend to use Fact 2.1 to prove Theorem 2.3. A trouble here is that $\mathcal{H}$ is not second countable in general. The following is a trick to avoid this trouble.

Lemma 2.13. Let $\left\{H_{\alpha(j)}\right\}_{j \in \mathbb{N}}$ be a countable subnet of $\left\{H_{\alpha}\right\}_{\alpha \in \mathcal{A}}$. Then, the subspace $\mathcal{H}^{\prime}:=\bigsqcup_{j=1}^{\infty} H_{\alpha(j)} \sqcup H$ of $\mathcal{H}$ is second countable.

Proof. The separability of $H$ allows us to find a countable dense subset $\left\{u_{i}\right\}_{i \in \mathbb{N}}$ of $H$. For each $i$ there are $u_{i j} \in H_{\alpha(j)}, j \in \mathbb{N}$, tending to $u_{i}$ as $j \rightarrow \infty$. For any $i, j, k \in \mathbb{N}$ we set

$$
O_{i j k}:=B\left(u_{i}, 1 / k\right) \cup \bigcup_{j^{\prime}=j}^{\infty} B\left(u_{i j^{\prime}}, 1 / k\right) \subset \mathcal{H}^{\prime},
$$

where $B(u, r):=\left\{v \in H_{\nu} \mid\|u-v\|_{H_{\nu}}<r\right\}$ is the metric ball for $u \in$ $H_{\nu}, r>0$. Obviously, $O_{i j k}$ is an open neighborhood of $u_{i}$ in $\mathcal{H}^{\prime}$. We take a countable basis $\mathcal{O}_{j}$ of open subsets of each $H_{\alpha(j)}$. Let us prove that $\left\{O_{i j k}\right\}_{i, j, k \in \mathbb{N}} \cup \bigcup_{j \in \mathbb{N}} \mathcal{O}_{j}$ is a countable basis of open subsets of $\mathcal{H}^{\prime}$. Assume that $u \in O_{i_{1} j_{1} k_{1}} \cap O_{i_{2} j_{2} k_{2}} \cap H$. It suffices to prove that there are $i_{3}, j_{3}, k_{3} \in \mathbb{N}$ such that

$$
u \in O_{i_{3} j_{3} k_{3}} \subset O_{i_{1} j_{1} k_{1}} \cap O_{i_{2} j_{2} k_{2}} .
$$

Since $\left\|u-u_{i_{p}}\right\|_{H}<1 / k_{p}$ for $p=1,2$, we find a number $k_{3} \in \mathbb{N}$ such that

$$
\left\|u-u_{i_{p}}\right\|_{H}+1 / k_{3}<1 / k_{p}, \quad p=1,2,
$$

and then find a number $i_{3} \in \mathbb{N}$ such that

$$
\begin{aligned}
\left\|u-u_{i_{3}}\right\|_{H} & <1 / k_{3}, \\
\left\|u_{i_{3}}-u_{i_{p}}\right\|_{H}+1 / k_{3} & <1 / k_{p}, \quad p=1,2 .
\end{aligned}
$$


There exists $j_{3} \in \mathbb{N}$ such that for any $j \geq j_{3}$ and $p=1,2$,

$$
\left\|u_{i_{3} j}-u_{i_{p} j}\right\|_{H_{\alpha(j)}}+1 / k_{3}<1 / k_{p}
$$

The inequalities (2.12), (2.13), (2.14), and triangle inequality together imply (2.11). This completes the proof.

Lemma 2.14. Let $\left\{F_{\alpha}: H_{\alpha} \rightarrow \overline{\mathbb{R}}\right\}_{\alpha \in \mathcal{A}}$ be a net of functions. Then some subnet of it $\Gamma$-converges in the sense of Definition 2.8 .

Proof. We take a countable subnet $\left\{H_{\alpha(j)}\right\}_{j \in \mathbb{N}}$ of $\left\{H_{\alpha}\right\}_{\alpha \in \mathcal{A}}$ and set $\mathcal{H}^{\prime}:=$ $\bigsqcup_{j=1}^{\infty} H_{\alpha(j)} \sqcup H$. Extend $F_{\alpha(j)}$ to $F_{\alpha(j)}: \mathcal{H}^{\prime} \rightarrow \overline{\mathbb{R}}$ by setting $F_{\alpha(j)}:=\infty$ on $\mathcal{H}^{\prime} \backslash H_{\alpha(j)}$. Then, Lemma 2.13 and Fact 2.1 together show that $\left\{F_{\alpha(j)}\right\}_{j \in \mathbb{N}}$ has a subnet which $\Gamma$-converges in $\mathcal{H}^{\prime}$ to some function $F: \mathcal{H}^{\prime} \rightarrow \mathbb{R}$. By Lemma 2.12, $\left.F\right|_{H_{\alpha(j)}} \Gamma$-converges to $\left.F\right|_{H}$ in the sense of Definition 2.8. This completes the proof.

Proof of Theorem 2.3. The theorem follows from Lemmas 2.14 and 2.11.

The following definitions are originally due to Mosco [42] for a fixed Hilbert space. Let $\left\{\mathcal{E}_{\alpha}\right\}_{\alpha \in \mathcal{A}}$ be a net of closed quadratic forms $\mathcal{E}_{\alpha}$ on $H_{\alpha}$ and $\mathcal{E}$ a closed quadratic form on $H$.

Definition 2.11 (Mosco topology). We say that $\left\{\mathcal{E}_{\alpha}\right\}_{\alpha \in \mathcal{A}}$ Mosco converges to $\mathcal{E}$ if both (F2) and the following (F1') hold:

(F1') If a net $\left\{u_{\alpha}\right\}_{\alpha \in \mathcal{A}}$ with $u_{\alpha} \in H_{\alpha}$ weakly converges to a $u \in H$, then

$$
\mathcal{E}(u) \leq \frac{\lim }{\alpha} \mathcal{E}_{\alpha}\left(u_{\alpha}\right) \text {. }
$$

It follows that the Mosco convergence induces a topology, say the Mosco topology on $\mathcal{F}(\mathcal{H})$.

Clearly, a Mosco convergence implies the $\Gamma$-convergence, so that the Mosco topology is stronger than the $\Gamma$-topology. The Mosco topology is Hausdorff if $\left\{H_{\nu}\right\}_{\nu \in \mathcal{N}}$ is Hausdorff.

Definition 2.12 (Asymptotic compactness). The net $\left\{\mathcal{E}_{\alpha}\right\}_{\alpha \in \mathcal{A}}$ is said to be asymptotically compact if for any net $\left\{u_{\alpha}\right\}_{\alpha \in \mathcal{A}}$ such that $u_{\alpha} \in H_{\alpha}$ and $\varlimsup_{\alpha}\left(\mathcal{E}_{\alpha}\left(u_{\alpha}\right)+\left\|u_{\alpha}\right\|_{H_{\alpha}}^{2}\right)<\infty$, there exists a strongly convergent subnet of $\left\{u_{\alpha}\right\}_{\alpha \in \mathcal{A}}$. 
It is easy to see the following (cf. Lemma 2.3.2 of [42]):

Lemma 2.15. Assume that $\left\{\mathcal{E}_{\alpha}\right\}_{\alpha \in \mathcal{A}}$ is asymptotically compact. Then, $\left\{\mathcal{E}_{\alpha}\right\}_{\alpha \in \mathcal{A}} \Gamma$-converges to $\mathcal{E}$ if and only if $\left\{\mathcal{E}_{\alpha}\right\}_{\alpha \in \mathcal{A}}$ Mosco converges to $\mathcal{E}$.

Definition 2.13. We say that $\mathcal{E}_{\alpha} \rightarrow \mathcal{E}$ compactly if $\mathcal{E}_{\alpha} \rightarrow \mathcal{E}$ with respect to the Mosco topology and if $\left\{\mathcal{E}_{\alpha}\right\}_{\alpha \in \mathcal{A}}$ is asymptotically compact.

Theorem 2.3 and Lemma 2.15 together imply:

Corollary 2.3. If $\left\{\mathcal{E}_{\alpha}\right\}_{\alpha \in \mathcal{A}}$ is asymptotically compact, it has a compact convergent subnet.

\subsection{Convergence of spectral structures.}

A spectral structure on a Hilbert space $H$ over $K$ is defined to be a compatible set

$$
\Sigma=\left(A, \mathcal{E}, E,\left\{T_{t}\right\},\left\{R_{\zeta}\right\}\right),
$$

where $A$ is a selfadjoint nonnegative definite linear operator on $H$, which is considered as an infinitesimal generator associated with a densely defined closed quadratic form $\mathcal{E}$ (determined by $\mathcal{D}(\mathcal{E})=\mathcal{D}(\sqrt{A})$ and $\mathcal{E}(u, v)=$ $\left.(\sqrt{A} u, \sqrt{A} v)_{H}, u, v \in \mathcal{D}(\mathcal{E})\right)$, a spectral measure $E$, a strongly continuous contraction semigroup $\left\{T_{t}\right\}_{t \geq 0}\left(T_{t}=e^{-t A}, t \geq 0\right)$, and with a strongly continuous resolvent $\left\{R_{\zeta}\right\}_{\zeta \in \rho(A)}\left(R_{\zeta}=(\zeta-A)^{-1}, \zeta \in \rho(A)\right)$, where $\rho(A)$ denotes the resolvent set of $A$.

Throughout this and the next section, let

$$
\Sigma=\left(A, \mathcal{E}, E,\left\{T_{t}\right\},\left\{R_{\zeta}\right\}\right), \quad \Sigma_{\alpha}=\left(A_{\alpha}, \mathcal{E}_{\alpha}, E_{\alpha},\left\{T_{t}^{\alpha}\right\},\left\{R_{\zeta}^{\alpha}\right\}\right)
$$

be given spectral structures on $H$ and $H_{\alpha}$ respectively.

Theorem 2.4. The following are all equivalent:

(1) $\mathcal{E}_{\alpha} \rightarrow \mathcal{E}$ with respect to the Mosco topology (resp. $\mathcal{E}_{\alpha} \rightarrow \mathcal{E}$ compactly).

(2) $R_{\zeta}^{\alpha} \rightarrow R_{\zeta}$ strongly (resp. compactly) for some $\zeta<0$.

(3) $T_{t}^{\alpha} \rightarrow T_{t}$ strongly (resp. compactly) for some $t>0$.

(4) $\varphi\left(A_{\alpha}\right) \rightarrow \varphi(A)$ strongly (resp. compactly) for any continuous function $\varphi:[0, \infty) \rightarrow K$ with compact support. 
(5) $\varphi_{\alpha}\left(A_{\alpha}\right) \rightarrow \varphi(A)$ strongly (resp. compactly) for any net $\left\{\varphi_{\alpha}:[0, \infty) \rightarrow\right.$ $K\}$ of continuous functions vanishing at infinity which uniformly converges to a continuous function $\varphi:[0, \infty) \rightarrow K$ vanishing at infinity.

(6) $E_{\alpha}((\lambda, \mu]) \rightarrow E((\lambda, \mu])$ strongly (resp. compactly) for any two real numbers $\lambda<\mu$ which are not in the point spectrum of $A$.

(7) $\left(E_{\alpha} u_{\alpha}, v_{\alpha}\right)_{H_{\alpha}} \rightarrow(E u, v)_{H}$ vaguely for any nets $\left\{u_{\alpha}\right\}_{\alpha \in \mathcal{A}},\left\{v_{\alpha}\right\}_{\alpha \in \mathcal{A}}$ of vectors $u_{\alpha}, v_{\alpha} \in H_{\alpha}$ and any $u, v \in H$ such that $u_{\alpha} \rightarrow u$ strongly and $v_{\alpha} \rightarrow v$ weakly (resp. $u_{\alpha} \rightarrow u$ weakly and $v_{\alpha} \rightarrow v$ weakly).

Proof. The equivalence between (2)-(7) is obtained in the same way as in the proof of Theorem 2.1.

The equivalence between (1) and (2) for the Mosco/strong topology is proved in the same way as in the proof of Theorem 2.4.1 of [42]. (Although the definition of the resolvent $G_{\zeta}:=(\zeta+A)^{-1}, \zeta \in \rho(-A)$, in [42] is different from our resolvent $R_{\zeta}=(\zeta-A)^{-1}$, it can be translated by $R_{\zeta}=-G_{-\zeta}$.)

Let us prove $(6) \Longrightarrow(1)$ for the compact topologies. It suffices to show the asymptotic compactness of $\left\{\mathcal{E}_{\alpha}\right\}$. Assume that

$$
\sup _{\alpha}\left(\mathcal{E}_{\alpha}\left(u_{\alpha}\right)+\left\|u_{\alpha}\right\|_{H_{\alpha}}^{2}\right) \leq M<\infty .
$$

Replacing with a subnet of $\mathcal{A}$, we assume that $u_{\alpha} \rightarrow u$ weakly. Let $\rho>0$ be a number which is not in the point spectrum of $A$. Since

$$
\int_{(\rho, \infty)} d\left(E_{\alpha} u_{\alpha}, u_{\alpha}\right)_{H_{\alpha}} \leq \frac{1}{\rho} \int_{(\rho, \infty)} \lambda d\left(E_{\alpha}(\lambda) u_{\alpha}, u_{\alpha}\right)_{H_{\alpha}} \leq \frac{\mathcal{E}_{\alpha}\left(u_{\alpha}\right)}{\rho} \leq \frac{M}{\rho}
$$

we have

$$
\left\|u_{\alpha}\right\|_{H_{\alpha}}^{2} \leq \int_{[0, \rho]} d\left(E_{\alpha} u_{\alpha}, u_{\alpha}\right)_{H_{\alpha}}+\frac{M}{\rho}
$$

and hence, by (6),

$$
\varlimsup_{\alpha}\left\|u_{\alpha}\right\|_{H_{\alpha}}^{2} \leq \int_{[0, \rho]} d(E u, u)_{H}+\frac{M}{\rho} \leq\|u\|_{H}^{2}+\frac{M}{\rho} .
$$

Since $\rho>0$ can be taken to be arbitrarily large, we obtain

$$
\varlimsup_{\alpha}\left\|u_{\alpha}\right\|_{H_{\alpha}} \leq\|u\|_{H}
$$

which shows that $u_{\alpha} \rightarrow u$ strongly. Thus, $\left\{\mathcal{E}_{\alpha}\right\}$ is asymptotically compact. 
Let us prove $(1) \Longrightarrow(3)$ for the compact topologies. Fix a number $t>0$, and let $\left\{u_{\alpha}\right\}$ be a net of vectors $u_{\alpha} \in H_{\alpha}$ weakly converging to a vector $u \in H$. We have already proved $T_{t}^{\alpha} \rightarrow T_{t}$ strongly. Then, by Lemma 2.6(1), $T_{t}^{\alpha} u_{\alpha} \rightarrow T_{t} u$ weakly. By setting $M:=\sup _{\alpha}\left\|u_{\alpha}\right\|_{H_{\alpha}}$, it follows that

$$
\begin{aligned}
\mathcal{E}_{\alpha}\left(T_{t}^{\alpha} u_{\alpha}\right) & =\int_{[0, \infty)} \lambda e^{-2 \lambda t} d\left(E_{\alpha}(\lambda) u_{\alpha}, u_{\alpha}\right)_{H_{\alpha}} \\
& \leq \int_{[0, \infty)} \frac{1-e^{-2 \lambda t}}{2 t} d\left(E_{\alpha}(\lambda) u_{\alpha}, u_{\alpha}\right)_{H_{\alpha}} \\
& =\frac{\left\|u_{\alpha}\right\|_{H_{\alpha}}^{2}-\left\|T_{t}^{\alpha} u_{\alpha}\right\|_{H_{\alpha}}^{2}}{2 t} \leq \frac{M}{2 t}
\end{aligned}
$$

and

$$
\sup _{\alpha}\left\|T_{t}^{\alpha} u_{\alpha}\right\| \leq M
$$

Hence, the asymptotic compactness of $\left\{\mathcal{E}_{\alpha}\right\}$ shows that $T_{t}^{\alpha} u_{\alpha} \rightarrow T_{t} u$ strongly by replacing with a subnet of $\mathcal{A}$. This completes the proof.

Definition 2.14. If one (or all) of the conditions in Theorem 2.4 holds, we say that $\left\{\Sigma_{\alpha}\right\}_{\alpha \in \mathcal{A}}$ strongly (resp. compactly) converges to $\Sigma$. Denote by $\mathcal{S}(\mathcal{H})$ the set of all spectral structures on $H_{\nu}, \nu \in \mathcal{N}$, and by $\mathcal{S}_{c}(\mathcal{H})$ the set of all $\Sigma \in$ $\mathcal{S}(\mathcal{H})$ which has compact resolvent. The strong (resp. compact) convergence induces a topology on $\mathcal{S}(\mathcal{H})$ (resp. $\mathcal{S}_{c}(\mathcal{H})$ ), say the strong (resp. compact) spectral topology.

Remark 2.6. If $\Sigma_{\alpha} \rightarrow \Sigma$ compactly, then $R_{\zeta}, T_{t}$, and $E((\lambda, \mu])$ are all compact operators by Lemma 2.7 . Therefore, if $R_{\zeta}$ is not compact, $\Sigma_{\alpha}:=\Sigma$ cannot compactly converge to $\Sigma$. Thus, the compact convergence does not induces a topology on the set of all spectral structures on $H_{\nu}, \nu \in \mathcal{N}$, in general.

For a real Hilbert space $H$ we have the complex Hilbert space $\hat{H}:=H \otimes \mathbb{C}$. Conversely, for a given complex Hilbert space $\hat{H}$ we have the real Hilbert space $H$ with $\hat{H}=H \otimes \mathbb{C}$. There is a $1-1$ correspondence between selfadjoint operators $A$ on $H$ and selfadjoint operators $\hat{A}$ on $\hat{H}$ such that $\hat{A}(u+i v)=$ $A u+i A v$ for $u, v \in H$, or $\left.\hat{A}\right|_{H}=A$. Of-course, $A$ is nonnegative if and only if so is $\hat{A}$. Assume that $H_{\alpha}, H$ are real Hilbert spaces, and $\hat{\Sigma}_{\alpha}, \hat{\Sigma}$ the spectral structures associated with nonnegative selfadjoint operators $\hat{A}_{\alpha}, \hat{A}$ on $\hat{H}_{\alpha}, \hat{H}$ respectively. We obviously have the following:

Proposition 2.4. The following are all equivalent: 
(1) $\Sigma_{\alpha} \rightarrow \Sigma$ strongly (resp. compactly).

(2) $\hat{\Sigma}_{\alpha} \rightarrow \hat{\Sigma}$ strongly (resp. compactly).

(3) One of the conditions in Theorem 2.1 for $\hat{A}, \hat{A}_{\alpha}$ holds.

Theorem 2.2 and Proposition 2.4 together imply:

Theorem 2.5. For a fixed $K:=\mathbb{R}$ or $\mathbb{C}$, let $H_{\alpha}, H$ have coefficient field $K$. The following are all equivalent:

(1) $\Sigma_{\alpha} \rightarrow \Sigma$ strongly.

(2) $\varphi_{\alpha}\left(A_{\alpha}\right) \rightarrow \varphi(A)$ strongly for any net $\left\{\varphi_{\alpha}:[0, \infty) \rightarrow K\right\}$ of bounded continuous functions uniformly converging to a bounded continuous function $\varphi:[0, \infty) \rightarrow K$.

(3) The strong graph limit $\Gamma_{\infty}$ of $\left\{A_{\alpha}\right\}_{\alpha \in \mathcal{A}}$ coincides with the graph of $A$.

Remark 2.7. We should notice that, although the statements of Theorems 2.1-2.5 and Proposition 2.4 are similar to those of the well-known theorems for a fixed Hilbert space (cf. [36, 42, 47, 48]), yet they are never exactly the same even if we assume $H_{\alpha}=H$ for all $\alpha \in \mathcal{A}$. This is because of the difference of the definition of the strong convergence of operators. Our case is a little more delicate. Besides, the statement for compact convergence is quite new.

As a consequence of Theorem 2.4, we have the following well-known statement. The proof is omitted.

Corollary 2.4. The following are equivalent:

(1) The embedding $\left(\mathcal{D}(\mathcal{E}), \mathcal{E}_{1}\right) \hookrightarrow H$ is compact.

(2) $R_{\zeta}$ is compact for some $\zeta \in \rho(A)$.

(3) $T_{t}$ is compact for some $t>0$.

(4) $\varphi(A)$ is compact for any continuous function $\varphi: \sigma(A) \rightarrow K$ vanishing at infinity.

(5) $E((\lambda, \mu])$ is compact for any two real numbers $\lambda<\mu$ which are not in the point spectrum of $A$.

(6) $\left(E u_{\alpha}, v_{\alpha}\right)_{H} \rightarrow(E u, v)_{H}$ vaguely for any nets $\left\{u_{\alpha}\right\}_{\alpha \in \mathcal{A}},\left\{v_{\alpha}\right\}_{\alpha \in \mathcal{A}} \subset H$ such that $u_{\alpha} \rightarrow u$ and $v_{\alpha} \rightarrow v$ weakly. 


\subsection{Asymptotic behavior of spectra.}

Denote by $\sigma(\cdot)$ the spectrum of an operator.

Proposition 2.5. If $\Sigma_{\alpha} \rightarrow \Sigma$ strongly, then

$$
\sigma(A) \subset \lim _{\alpha \in \mathcal{A}} \sigma\left(A_{\alpha}\right)
$$

i.e., for any $\lambda \in \sigma(A)$ there exist $\lambda_{\alpha} \in \sigma\left(A_{\alpha}\right)$ tending to $\lambda$.

Proof. We prove the proposition in the same way as in the proof of Theorem 1.14 in VIII $\S 2$ of [36]. By Proposition 2.4 and since $\sigma(A)=\sigma(\hat{A})$, we may assume that $H$ and $H_{\alpha}$ are all complex Hilbert spaces.

Take any $\lambda \in \sigma(A)$ and fix it. For a number $\epsilon>0$ we set $\zeta:=\lambda+i \epsilon$. Then,

$$
\left\|R_{\zeta}^{\alpha}\right\|_{L\left(H_{\alpha}\right)}=\frac{1}{\inf _{\rho \in \sigma\left(A_{\alpha}\right)}|\zeta-\rho|}, \quad \text { and } \quad\left\|R_{\zeta}\right\|_{L(H)}=\frac{1}{\inf _{\rho \in \sigma(A)}|\zeta-\rho|}=\frac{1}{\epsilon} .
$$

Applying Theorem 2.1(3) yields that $R_{\zeta}^{\alpha} \rightarrow R_{\zeta}$ strongly. Hence, by Lemma 2.9 ,

$$
\varlimsup_{\alpha} \inf _{\rho \in \sigma\left(A_{\alpha}\right)}|\zeta-\rho| \leq \epsilon
$$

Since $\epsilon>0$ is arbitrary, this completes the proof.

Remark 2.8. The conclusion of Proposition 2.5 actually holds even if $A$ and $A_{\alpha}$ are not necessarily nonnegative and if one (or all) of the conditions in Theorem 2.1 holds.

Lemma 2.16. If two extended real numbers $a, b$ with $-\infty \leq a<b \leq \infty$ are both not in the point spectrum of $A$, we have

$$
a \leq \frac{\mathcal{E}(u)}{\|u\|_{H}^{2}} \leq b \quad \text { for any } u \in E((a, b]) H \backslash\{o\} .
$$

Here we agree that $E((a, b])=E((a, \infty))$ for $b=\infty$.

Proof. Let $a<b$ be not in the point spectrum of $A$ and let $u \in E((a, b]) H \backslash$ $\{o\}$. Then,

$$
\int_{(a, b]} d E u=E((a, b]) u=u=\int_{\mathbb{R}} d E u
$$


and hence $(E u, u)=0$ on $\mathbb{R} \backslash(a, b]$. Therefore, if $u \in \mathcal{D}(A)$,

$$
\mathcal{E}(u)=(A u, u)_{H}=\int_{\mathbb{R}} \lambda d(E(\lambda) u, u)_{H}=\int_{(a, b]} \lambda d(E(\lambda) u, u)_{H}
$$

which is not less than $a \int_{(a, b]} d(E u, u)_{H}=a\|u\|_{H}^{2}$ and not greater than $b \int_{(a, b]} d(E u, u)_{H}=b\|u\|_{H}^{2}$. This completes the proof.

Define $n(I):=\operatorname{dim} E(I) H$ and $n_{\alpha}(I):=\operatorname{dim} E_{\alpha}(I) H$ for a Borel subset $I \subset \mathbb{R}$.

Proposition 2.6. Let $a<b$ be two numbers which are not in the point spectrum of $A$. If $\Sigma_{\alpha} \rightarrow \Sigma$ strongly, we have

$$
\varliminf_{\alpha} n_{\alpha}((a, b]) \geq n((a, b])
$$

and in particular,

$$
\frac{\lim }{\alpha} \operatorname{dim} H_{\alpha} \geq \operatorname{dim} H
$$

Proof. Take a complete orthonormal basis $\left\{\varphi_{k}\right\}_{k=1}^{n((a, b])}$ of $E((a, b]) H$. Let $n \in \mathbb{N}$ be any fixed number if $n((a, b])=\infty$, and $n:=n((a, b])$ if $n((a, b])<$ $\infty$. There are $\varphi_{k}^{\alpha} \in H_{\alpha}, k=1, \ldots, n$, with $\lim _{\alpha} \varphi_{k}^{\alpha}=\varphi_{k}$. Since $E_{\alpha}((a, b]) \rightarrow$ $E((a, b])$ strongly, setting $\psi_{k}^{\alpha}:=E_{\alpha}((a, b]) \varphi_{k}^{\alpha} \in E_{\alpha}((a, b]) H_{\alpha}$ we have

$$
\lim _{\alpha} \psi_{k}^{\alpha}=E((a, b]) \varphi_{k}=\varphi_{k}
$$

and so

$$
\lim _{\alpha}\left(\psi_{k}^{\alpha}, \psi_{\ell}^{\alpha}\right)_{H_{\alpha}}=\left(\varphi_{k}, \varphi_{\ell}\right)_{H}=\delta_{k \ell} .
$$

This proves that $\left\{\psi_{k}^{\alpha}\right\}_{k=1}^{n}$ is linearly independent for all sufficiently large $\alpha$ and hence $\lim _{\alpha} n_{\alpha}((a, b]) \geq n$. This proves (2.15). Since $n((a, b]) \rightarrow \operatorname{dim} H$ as $a \rightarrow-\infty, b \rightarrow \infty$, we obtain (2.16).

Theorem 2.6. Assume that $\Sigma_{\alpha} \rightarrow \Sigma$ compactly. Then, for any $a, b \in$ $\mathbb{R} \backslash \sigma(A)$ with $a<b$, we have $n_{\alpha}((a, b])=n((a, b])$ for sufficiently large $\alpha$. In particular, the limit set of $\sigma\left(A_{\alpha}\right)$ coincides with $\sigma(A)$.

Remark 2.9. Under the assumption of this theorem, Lemma 2.7 implies that $R_{\zeta}, T_{t}$, and $E((\lambda, \mu])$ are all compact operators, so that $A$ has only discrete spectrum and $n((a, b])<\infty$ for any $a<b<\infty$. 
Proof of Theorem 2.6. The spectrum $\sigma(A)$ of $A$ is a finite or infinite sequence of eigenvalues $(0 \leq) \lambda_{1} \leq \lambda_{2} \leq \cdots \leq \lambda_{n}, n \in\{0\} \cup \mathbb{N} \cup\{\infty\}$, with no accumulation, where $n=0$ means that it is the empty sequence, and $n=\infty$ means that it is an infinite sequence tending to infinity. We set $L_{1}^{\alpha}:=$ $E_{\alpha}\left(\left(-\infty, \lambda_{1}+\epsilon_{0}\right]\right) H_{\alpha}$ and $L_{1}:=H$ for a fixed $\epsilon_{0}>0$, where $\lambda_{1}+\epsilon_{0}=\lambda_{1}:=$ $\infty$ if $n=0$. Let

$$
\mu_{1}:=\underline{\lim } \inf \left\{\mathcal{E}_{\alpha}(u) \mid\|u\|_{H_{\alpha}}=1, u \in L_{1}^{\alpha}\right\}
$$

Then, by Lemma 2.16, we have $\underline{\lim }_{\alpha} n_{\alpha}((-\infty, \mu])=0$ for any $\mu \in\left(-\infty, \mu_{1}\right)$, which together with Proposition 2.6 shows $\mu_{1} \leq \lambda_{1}$. Therefore, when $\mu_{1}=\infty$, we have $n=0$ and $H_{\alpha}=o$ for sufficiently large $\alpha$, which implies the theorem. Suppose $\mu_{1}<\infty$. There exist unit vectors $\varphi_{1}^{\alpha} \in L_{1}^{\alpha}$ for all sufficiently large $\alpha$ such that $\underline{\lim }_{\alpha} \mathcal{E}_{\alpha}\left(\varphi_{1}^{\alpha}\right)=\mu_{1}$. Since $\mathcal{E}_{\alpha} \rightarrow \mathcal{E}$ compactly and by replacing with a subnet of $\mathcal{A}$, there exists a strong limit $\varphi_{1}:=\lim _{\alpha} \varphi_{1}^{\alpha} \in L_{1}$ with $\mathcal{E}\left(\varphi_{1}\right) \leq \mu_{1}$. It follows that $\left\|\varphi_{1}\right\|_{H}=1$ and $\lambda_{1}=\inf \left\{\mathcal{E}(u) \mid u \in L_{1},\|u\|_{H}=1\right\} \leq \mathcal{E}\left(\varphi_{1}\right) \leq \mu_{1}<\infty$. Thus, we obtain $n \geq 1, \lambda_{1}=\mu_{1}=\mathcal{E}\left(\varphi_{1}\right)$, and that $\varphi_{1}$ is a unit eigenvector for $\lambda_{1}$ of $A$. Since $E_{\alpha}\left(\left(\lambda_{1}-\epsilon, \lambda_{1}+\epsilon\right]\right) \rightarrow E\left(\left(\lambda_{1}-\epsilon, \lambda_{1}+\epsilon\right]\right)$ strongly for any fixed $\epsilon>0$ and since $E\left(\left(\lambda_{1}-\epsilon, \lambda_{1}+\epsilon\right]\right) \rightarrow E\left(\left\{\lambda_{1}\right\}\right)$ strongly as $\epsilon \searrow 0$, there exists a net of positive numbers $\epsilon_{1}^{\alpha} \rightarrow 0$ such that $E_{\alpha}\left(\left(\lambda_{1}-\epsilon_{1}^{\alpha}, \lambda_{1}+\epsilon_{1}^{\alpha}\right]\right) \rightarrow E\left(\left\{\lambda_{1}\right\}\right)$ strongly. Then we have $\psi_{1}^{\alpha}:=E_{\alpha}\left(\left(\lambda_{1}-\epsilon_{1}^{\alpha}, \lambda_{1}+\epsilon_{1}^{\alpha}\right]\right) \varphi_{1}^{\alpha} \rightarrow E\left(\left\{\lambda_{1}\right\}\right) \varphi_{1}=\varphi_{1}$.

We next set $L_{2}^{\alpha}:=E_{\alpha}\left(\left(-\infty, \lambda_{2}+\epsilon_{0}\right]\right) H_{\alpha} \cap\left\langle\psi_{1}^{\alpha}\right\rangle^{\perp}, L_{2}:=\left\langle\varphi_{1}\right\rangle^{\perp}$, and

$$
\mu_{2}:=\underline{\lim } \inf \left\{\mathcal{E}_{\alpha}(u) \mid\|u\|_{H_{\alpha}}=1, u \in L_{2}^{\alpha}\right\} .
$$

Then, by Lemma 2.16, we have $\underline{\lim }_{\alpha} n_{\alpha}((-\infty, \mu])=1$ for any $\mu \in\left(\mu_{1}, \mu_{2}\right)$, which together with Proposition 2.6 shows $\mu_{2} \leq \lambda_{2}$. Therefore, when $\mu_{2}=$ $\infty$, we have $n=1$ and $H_{\alpha}=\left\langle\psi_{1}^{\alpha}\right\rangle$ for sufficiently large $\alpha$. Suppose $\mu_{2}<\infty$. We take unit vectors $\varphi_{2}^{\alpha} \in L_{2}^{\alpha}$ such that $\underline{\lim }_{\alpha} \mathcal{E}_{\alpha}\left(\varphi_{2}^{\alpha}\right)=\mu_{2}$. Then, the same discussion as above yields $n \geq 2, \lambda_{2}=\mu_{2}$, and that $\varphi_{2}^{\alpha}$ strongly converges to a unit eigenvector $\varphi_{2}$ for $\lambda_{2}$ of $A$, by replacing with a subnet of $\mathcal{A}$. For some net of positive numbers $\epsilon_{2}^{\alpha} \rightarrow 0$, we have $\psi_{2}^{\alpha}:=E_{\alpha}\left(\left(\lambda_{2}-\epsilon_{2}^{\alpha}, \lambda_{2}+\epsilon_{2}^{\alpha}\right]\right) \varphi_{2}^{\alpha} \rightarrow$ $\varphi_{2}$. We see that for any $\epsilon>0$ there exists $\alpha_{\epsilon} \in \mathcal{A}$ such that for any $\alpha \geq \alpha_{\epsilon}$,

(1) $\psi_{i}^{\alpha} \in E_{\alpha}\left(\left(\lambda_{i}-\epsilon, \lambda_{i}+\epsilon\right]\right) H_{\alpha}$ for each $i=1,2$,

(2) if $\lambda_{1}+2 \epsilon<\lambda_{2}$ then

$$
E_{\alpha}\left(\left(\lambda_{1}-\epsilon, \lambda_{1}+\epsilon\right]\right) H_{\alpha}=\left\langle\psi_{1}^{\alpha}\right\rangle \quad \text { and } \quad E_{\alpha}\left(\left(\lambda_{1}+\epsilon, \lambda_{2}-\epsilon\right]\right) H_{\alpha}=o .
$$


We repeat this procedure. Setting $L_{k}^{\alpha}:=E_{\alpha}\left(\left(-\infty, \lambda_{k}+\epsilon_{0}\right]\right) H_{\alpha} \cap$ $\left\langle\psi_{1}, \ldots, \psi_{k-1}\right\rangle^{\perp}$, we have

$$
\lambda_{k}=\mu_{k}:=\underline{\lim } \inf \left\{\mathcal{E}_{\alpha}(u) \mid\|u\|_{H_{\alpha}}=1, u \in L_{k}^{\alpha}\right\}
$$

while $k \leq n$. Let $k=1,2, \ldots, n$ be any number and $\epsilon>0$ be sufficiently small compared with $k$. Then, there exists $\alpha_{k, \epsilon} \in \mathcal{A}$ such that for any $\alpha \geq \alpha_{k, \epsilon}$,

(1) for each $\lambda \in\left\{\lambda_{1}, \ldots, \lambda_{k-1}\right\}$ with $\lambda<\lambda_{k}$,

$$
E_{\alpha}((\lambda-\epsilon, \lambda+\epsilon]) H_{\alpha}=\left\langle\psi_{i}^{\alpha} \mid p_{\lambda} \leq i \leq q_{\lambda}\right\rangle,
$$

where $p_{\lambda}:=\min \left\{i \in \mathbb{N} \mid \lambda_{i}=\lambda\right\}$ and $q_{\lambda}:=\max \left\{i \in \mathbb{N} \mid \lambda_{i}=\lambda\right\}$,

(2) for each $i=1, \ldots, k-1$ with $\lambda_{i}<\lambda_{i+1}$,

$$
E_{\alpha}\left(\left(\lambda_{i}+\epsilon, \lambda_{i+1}-\epsilon\right]\right) H_{\alpha}=o .
$$

Let $a, b \in[0, \infty) \backslash \sigma(A)$ be two given numbers with $a<b$. The above implies that

$$
\left.E_{\alpha}((a, b]) H_{\alpha}=\left\langle\psi_{k}^{\alpha}\right| k=1, \ldots, n \text { with } a<\lambda_{k} \leq b\right\rangle
$$

for all sufficiently large $\alpha$. Thus, $n_{\alpha}((a, b])$ coincides with the number of $k$ 's with $a<\lambda_{k} \leq b$, namely $n((a, b])$. This completes the proof.

Corollary 2.5. Assume that $\Sigma_{\alpha} \rightarrow \Sigma$ compactly and that the resolvents $R_{\zeta}^{\alpha}$ are all compact. Denote by $\lambda_{k}\left(\right.$ resp. $\left.\lambda_{k}^{\alpha}\right)$ the $k^{\text {th }}$ eigenvalue of $A\left(\right.$ resp. $\left.A_{\alpha}\right)$ with multiplicity. We set $\lambda_{k}:=\infty$ for all $k \geq \operatorname{dim} H+1$ when $\operatorname{dim} H<\infty$, and $\lambda_{k}^{\alpha}:=\infty$ for all $k \geq \operatorname{dim} H_{\alpha}+1$ when $\operatorname{dim} H_{\alpha}<\infty$. Then we have

$$
\lim _{\alpha} \lambda_{k}^{\alpha}=\lambda_{k} \quad \text { for any } k .
$$

Moreover, let $\left\{\varphi_{k}^{\alpha}\right\}_{k=1}^{\operatorname{dim} H_{\alpha}}$ be an orthonormal basis on $H_{\alpha}$ such that $\varphi_{k}^{\alpha}$ is an eigenvector for $\lambda_{k}^{\alpha}$ of $A_{\alpha}$. Then, by replacing with a subnet of $\mathcal{A}$ if necessarily, for each fixed $k \in \mathbb{N}$ with $k \leq \operatorname{dim} H$, the vector $\varphi_{k}^{\alpha}$ strongly converges to some eigenvector $\varphi_{k}$ for $\lambda_{k}$ of $A$ such that $\left\{\varphi_{k}\right\}_{k=1}^{\operatorname{dim} H}$ is a complete orthonormal basis on $H$.

Proof. In the proof of Theorem 2.6, we can choose $\varphi_{k}^{\alpha}$ as in the statement of the corollary. Thus, the corollary follows from the discussion in the proof of Theorem 2.6. 
Remark 2.10. Throughout this section, we have assumed the separability of Hilbert spaces for the simplicity of proofs. However, this is not essential and all results except Theorem 2.3 still hold for non-separable Hilbert spaces.

\section{Convergence of manifolds.}

\subsection{Preliminaries for Lipschitz-Riemannian manifold.}

Let us first recall Lipschitz manifold (see [55] for more details). A Lipschitz manifold is defined to be a paracompact topological manifold with atlas $\left\{\left(U_{\lambda}, \varphi_{\lambda}\right)\right\}_{\lambda \in \Lambda}$ whose chart transformations $\varphi_{\mu} \circ \varphi_{\lambda}^{-1}: \varphi_{\lambda}\left(U_{\lambda} \cap U_{\mu}\right) \rightarrow \varphi_{\mu}\left(U_{\lambda} \cap\right.$ $\left.U_{\mu}\right)$ are bi-Lipschitz maps between open subsets of $\mathbb{R}^{n}$. A Riemannian metric on a Lipschitz manifold with atlas $\left\{\left(U_{\lambda}, \varphi_{\lambda}\right)\right\}_{\lambda \in \Lambda}$ is defined to be a family of measurable Riemannian metrics $g_{\lambda}$ on $\varphi_{\lambda}\left(U_{\lambda}\right) \subset \mathbb{R}^{n}$ for all $\lambda \in \Lambda$ which satisfies the two following conditions:

(1) the compatibility condition on chart transformations

$$
\left(\varphi_{\mu} \circ \varphi_{\lambda}^{-1}\right)^{*} g_{\mu}=g_{\lambda} \text { a.e. }
$$

(2) for each $\lambda \in \Lambda$ there exists a constant $c_{\lambda} \in(0,1)$ such that

$$
c_{\lambda}\|\omega\|_{L^{2}\left(\mathbb{R}^{n}\right)} \leq\|\omega\|_{L^{2}\left(g_{\lambda}\right)} \leq c_{\lambda}^{-1}\|\omega\|_{L^{2}\left(\mathbb{R}^{n}\right)}
$$

for any $C^{\infty}$ differential form $\omega$ on $\mathbb{R}^{n}$ with compact support in $\varphi_{\lambda}\left(U_{\lambda}\right)$, where $\|\cdot\|_{L^{2}\left(\mathbb{R}^{n}\right)}$ and $\|\cdot\|_{L^{2}\left(g_{\lambda}\right)}$ denote the $L^{2}$ norms with respect to the Euclidean metric and $g_{\lambda}$ respectively.

Note that (2) is required to define the $L^{2}$ norm of differential form on the manifold. We also define a Lipschitz manifold with boundary in an ordinary manner.

Let $M$ be a Lipschitz-Riemannian manifold (possibly with boundary), i.e., a Lipschitz manifold equipped with a Riemannian metric. Then, we have the distance function on $M$ induced from the Riemannian metric (see [20]). It follows that the $n$-dimensional Hausdorff measure and the Riemannian volume coincide for any Borel subset of $M$ (cf. $\$ 7.1$ of [45]). We define the $(1,2)$-Sobolev space $W^{1,2}(M)$ to be the set of real valued $L^{2}$ functions $u$ on $M$ admitting $L^{2}$ weak derivative $d u$. A canonical Dirichlet form $\mathcal{E}_{M}$ : $W^{1,2}(M) \times W^{1,2}(M) \rightarrow \mathbb{R}$ is defined by

$$
\mathcal{E}_{M}(u, v):=\int_{M}\langle d u, d v\rangle_{M} d \operatorname{vol}_{M}, \quad u, v \in W^{1,2}(M)
$$


where $\langle\cdot, \cdot\rangle_{M}$ and $d \mathrm{vol}_{M}$ respectively denote the inner product and volume measure induced from the Riemannian metric on $M$. A (Hilbert) inner product (resp. norm) on $W^{1,2}(M)$ is defined by $(\cdot, \cdot)_{W^{1,2}}=(\cdot, \cdot)_{L^{2}}+\mathcal{E}_{M}(\cdot, \cdot)$ (resp. $\left.\|\cdot\|_{W^{1,2}}^{2}=\|\cdot\|_{L^{2}}^{2}+\mathcal{E}_{M}(\cdot)\right)$. Denote by $W_{0}^{1,2}(M)$ the $W^{1,2}$ closure of the set of $W^{1,2}$ functions with compact support on $M$. A standard partitionof-unity argument using Theorem 3 in $\S 4.2 .1$ of [21] shows that the set of Lipschitz functions with compact support on $M$ is dense in $W_{0}^{1,2}(M)$. The canonical spectral structure $\Sigma(M)$ on $M$ is defined to be that induced from the symmetric bilinear form $\left(\mathcal{E}_{M}, W_{0}^{1,2}(M)\right)$, where we mean by $\left(\mathcal{E}_{M}, \mathcal{F}\right)$ the symmetric bilinear form $\mathcal{E}_{M}$ with domain restricted on $\mathcal{F} \subset W^{1,2}(M)$. Note that $\left(\mathcal{E}_{M}, W_{0}^{1,2}(M)\right)$ is a strongly local regular Dirichlet form in the sense of the abstract Dirichlet form theory (see $\S 5.1$ ). We call its infinitesimal generator the Laplacian $\Delta_{M}$ on $M$. Remark that if $M$ is a smooth Riemannian manifold, $W_{0}^{1,2}(M)$ coincides with the $W^{1,2}$ closure of the set $C_{0}^{\infty}(M)$ of smooth functions with compact support on $M$ and the Laplacian $\Delta_{M}$ defined here is the Friedrichs extension of the $C^{\infty}$ Laplacian defined on $C_{0}^{\infty}(M)$. Note that when $M$ has nonempty boundary, the eigenvalue problem $\Delta_{M} u=\lambda u, u \in \mathcal{D}\left(\Delta_{M}\right)$, means one under the free (or Neumann) boundary condition on $\partial M$. If we consider the interior $M^{\circ}:=M \backslash \partial M$, then the eigenvalue problem $\Delta_{M^{\circ}} u=\lambda u, u \in \mathcal{D}\left(\Delta_{M^{\circ}}\right)$ is corresponding to one under the Dirichlet boundary condition on $\partial M$.

Let $M$ and $N$ be two Lipschitz-Riemannian manifolds. By using the short-time asymptotic formula for heat kernel $([43])$, it is easy to prove that if a measurable map $f: M \rightarrow N$ with $f^{*} \operatorname{vol}_{N}=\operatorname{vol}_{M}$ induces an isomorphism between the spectral structures of $M$ and $N$, i.e., $\mathcal{D}\left(\Delta_{M}\right)=\{u \circ f \mid u \in$ $\left.\mathcal{D}\left(\Delta_{N}\right)\right\}$ and $\Delta_{M}(u \circ f)=\left(\Delta_{N} u\right) \circ f$ for any $u \in \mathcal{D}\left(\Delta_{N}\right)$, then $M$ and $N$ are isometric. In other words, each isomorphism class of $(X, p, m, \Sigma)$ for $(X, p, m) \in \mathcal{M}$ and a spectral structure $\Sigma$ on $L^{2}(X ; m)$ contains at most one $\left(M, p, \operatorname{vol}_{M}, \Sigma(M)\right)$ induced from a Lipschitz-Riemannian manifold $M$.

An $\epsilon$-almost isometry from $M$ to $N$ is defined to be a bi-Lipschitz homeomorphism $f: M \rightarrow N$ with $|\ln \operatorname{dil}(f)|+\left|\ln \operatorname{dil}\left(f^{-1}\right)\right| \leq \epsilon$, where $\operatorname{dil}(f)$ denotes the dilatation of $f$, i.e., the smallest Lipschitz constant of $f$. If $f: M \rightarrow N$ is an $\epsilon$-almost isometry, the push-forward measure $f_{*} \operatorname{vol}_{M}$ satisfies

$$
e^{-n \epsilon} \operatorname{vol}_{N} \leq f_{*} \operatorname{vol}_{M} \leq e^{n \epsilon} \operatorname{vol}_{N}
$$

where $n:=\operatorname{dim} M=\operatorname{dim} N$, and we consequently have

$$
\left|\left\|f_{*} u\right\|_{L^{2}}-\|u\|_{L^{2}}\right| \leq \theta_{n}(\epsilon)\|u\|_{L^{2}}
$$


for any $u \in L^{2}(M)$, and

$$
\left|\mathcal{E}_{N}\left(f_{*} u\right)-\mathcal{E}_{M}(u)\right| \leq \theta_{n}(\epsilon) \mathcal{E}_{M}(u)
$$

for any $u \in W^{1,2}(M)$ (cf. Theorem 4(ii) in 4.2.2 of [21] and its Remark), where $\theta_{n}$ is some function depending only on $n$ such that $\lim _{\epsilon \rightarrow 0} \theta_{n}(\epsilon)=0$. In other words, the push-forward $f_{*}$ is a $\theta_{n}(\epsilon)$-isometry with respect to the $L^{2}$ and $W^{1,2}$ norms.

The Lipschitz distance $d_{L}(M, N)$ between $M$ and $N$ is defined to be the infimum of $\epsilon>0$ such that an $\epsilon$-almost isometry from $M$ to $N$ exists. The topology on the set of isometry classes of Lipschitz-Riemannian manifolds induced from the Lipschitz distance $d_{L}$ is called the Lipschitz topology.

\subsection{Compact Lipschitz convergence and spectral structure.}

In the following, Lipschitz-Riemannian manifolds may have nonempty boundaries and we do not assume the completeness of them. Let us first present a trivial proposition.

Proposition 3.1. Let $\left\{M_{\alpha}\right\}_{\alpha \in \mathcal{A}}$ be a net of Lipschitz-Riemannian manifolds and $M$ a Lipschitz-Riemannian manifold such that the embedding of $W^{1,2}(M)$ into $L^{2}(M)$ is compact. If $\left\{M_{\alpha}\right\}_{\alpha \in \mathcal{A}}$ converges to $M$ with respect to the Lipschitz topology, then $\left(M_{\alpha}, \operatorname{vol}_{M_{\alpha}}\right)$ converges to $\left(M, \operatorname{vol}_{M}\right)$ with respect to the measured $G H$ topology, and the spectral structure $\Sigma\left(M_{\alpha}\right)$ compactly converges to $\Sigma(M)$. Consequently, the spectrum $\sigma\left(\Delta_{M_{\alpha}}\right)$ converges to $\sigma\left(\Delta_{M}\right)$.

Proof. By the definition of the Lipschitz topology, there is a net of $\epsilon_{\alpha}$-almost isometries $f_{\alpha}: M_{\alpha} \rightarrow M, \epsilon_{\alpha} \rightarrow 0$. It is clear from (3.1) that $\left(M_{\alpha}, \operatorname{vol}_{M_{\alpha}}\right)$ converges to $\left(M, \operatorname{vol}_{M}\right)$ with respect to the measured GH topology. In order to prove the compact convergence of spectral structures, it suffices to show that the canonical Dirichlet form $\mathcal{E}_{M_{\alpha}}$ on $L^{2}\left(M_{\alpha}\right)$ compactly converges to the canonical Dirichlet form $\mathcal{E}_{M}$ on $L^{2}(M)$. From (3.2) and (3.3), we easily verify (F1) and (F2) in $\S 2.5$. The asymptotic compactness of $\left\{\mathcal{E}_{M_{\alpha}}\right\}$ follows from the compactness of the embedding $W^{1,2}(M) \hookrightarrow L^{2}(M)$. This completes the proof.

Let us briefly discuss the compactness of the embedding $W^{1,2}(M) \hookrightarrow$ $L^{2}(M)$, which is obtained if one of the following holds:

(1) $M$ is a compact Lipschitz-Riemannian manifold (with boundary). 
(2) More generally, $M$ is covered by finitely many open subsets bi-Lipschitz homeomorphic to an open unit disk.

We see an example of $M$ satisfying (2) in the following:

Example 3.1 (Lipschitz cone manifold). Define a notion of (non-branched) Lipschitz cone manifold inductively as follows. A one-dimensional Lipschitz cone manifolds is, by definition, a one-dimensional Riemannian manifold (possibly with boundary). For $n \geq 2$, an $n$-dimensional Lipschitz cone manifold is defined to be a metric space $X$ such that any point in $X$ admits a neighborhood bi-Lipschitz homeomorphic to a neighborhood of the vertex in the Euclidean cone over an $(n-1)$-dimensional compact connected Lipschitz cone manifold. See [8] for the definition of Euclidean cone. Any $n$-dimensional Lipschitz cone manifold $X$ splits into an $n$-dimensional Lipschitz-Riemannian manifold, say the regular set, and the set of points which do not have neighborhoods bi-Lipschitz homeomorphic to an open subsets of $\mathbb{R}^{n}$, say the singular set. The singular set is of Hausdorff dimension $\leq n-1$. The regular set of a compact Lipschitz cone manifold satisfies the condition (2) above. Because, if $X$ is covered by finitely many open subsets bi-Lipschitz homeomorphic to an open unit disk, so is the $r$-ball at the vertex in the Euclidean cone over $X$ for each $r>0$.

Perelman claims that every Alexandrov space is a Lipschitz cone manifold, the proof of which is unpublished. Another proof of the compactness of the embedding $W^{1,2}(M) \hookrightarrow L^{2}(M)$ for compact Alexandrov space $M$ is seen in [38].

The purpose of this section is to extend the above proposition for manifolds which may have continuous spectra of Laplacian. We here define a new notion of convergence of manifolds.

Definition 3.1 (Compact Lipschitz topology). We say that a net $\left\{M_{\alpha}\right\}_{\alpha \in \mathcal{A}}$ of Lipschitz-Riemannian manifolds compact Lipschitz converges to a Lipschitz-Riemannian manifold $M$ if for any relatively compact open subset $O \subset M$ there exists a net of relatively compact open subsets $O_{\alpha} \subset M_{\alpha}$ such that $O_{\alpha}$ Lipschitz converges to $O$. This convergence induces a topology on the set of Lipschitz-Riemannian manifolds, say the compact Lipschitz topology.

Remark 3.1. Let $M$ be a Lipschitz-Riemannian manifold and $\left\{M_{\alpha}\right\}_{\alpha \in \mathcal{A}}$ a net of Lipschitz-Riemannian manifolds. 
(1) If $M_{\alpha}$ Lipschitz converges to $M$, then $M_{\alpha}$ compact Lipschitz converge to $M$. However, the converse is not true in general.

(2) If there exist open subsets $N_{\alpha} \subset M_{\alpha}$ which compact Lipschitz converges to $M$, then $M_{\alpha}$ compact Lipschitz converges to $M$. In particular, the compact Lipschitz topology is not Hausdorff.

(3) If $\left\{M_{\alpha}\right\}_{\alpha \in \mathcal{A}}$ is an increasing net of open subsets of $M$ with $\bigcup_{\alpha} M_{\alpha}=$ $M$, then $M_{\alpha}$ compact Lipschitz converges to $M$.

Theorem 1.1 is more precisely restated as follows:

Theorem 3.1. Let $\left\{M_{\alpha}\right\}$ be a net of Lipschitz-Riemannian manifolds and $M$ a Lipschitz-Riemannian manifold with $W^{1,2}(M)=W_{0}^{1,2}(M)$. If $M_{\alpha}$ compact Lipschitz converges to $M$, then $\left(M_{\alpha}, \operatorname{vol}_{M_{\alpha}}\right)$ converges to $\left(M, \operatorname{vol}_{M}\right)$ with respect to the measured compact $G H$ topology and the spectral structure $\Sigma\left(M_{\alpha}\right)$ strongly converges to $\Sigma(M)$, in particular

$$
\sigma\left(\Delta_{M}\right) \subset \lim _{\alpha} \sigma\left(\Delta_{M_{\alpha}}\right)
$$

Proof. The definition of the compact Lipschitz convergence leads to the existence of $\epsilon_{\alpha}$-almost isometries $f_{\alpha}: M_{\alpha} \supset O_{\alpha} \rightarrow f_{\alpha}\left(O_{\alpha}\right) \subset M, \epsilon_{\alpha} \searrow 0$, such that $O_{\alpha}$ and $f_{\alpha}\left(O_{\alpha}\right)$ for each $\alpha$ are both relatively compact open subsets and $\left\{f_{\alpha}\left(O_{\alpha}\right)\right\}_{\alpha}$ is a monotone increasing net covering $M$. We easily verify that $\left(M_{\alpha}, \operatorname{vol}_{M_{\alpha}}\right)$ converges to $\left(M, \operatorname{vol}_{M}\right)$ with respect to the measured compact GH topology. It suffices to show that the canonical Dirichlet form $\mathcal{E}_{M_{\alpha}}$ on $L^{2}\left(M_{\alpha}\right)$ converges to the canonical Dirichlet form $\mathcal{E}_{M}$ on $L^{2}(M)$ with respect to the Mosco topology. To verify (F1') in $\S 2.5$, we take a net $u_{\alpha} \in W^{1,2}\left(M_{\alpha}\right)$ with

$$
R:=\varlimsup_{\alpha}\left\|u_{\alpha}\right\|_{W^{1,2}\left(M_{\alpha}\right)}<\infty .
$$

For a relatively compact open subset $C \subset M$ with Lipschitz boundary, there exists $\alpha_{C} \in \mathcal{A}$ such that $C \subset f_{\alpha}\left(O_{\alpha}\right)$ for all $\alpha \geq \alpha_{C}$. Setting $\bar{u}_{\alpha}:=u_{\alpha} \circ$ $\left.f_{\alpha}^{-1}\right|_{C} \in W^{1,2}(C)$ for $\alpha \geq \alpha_{C}$, we have

$$
\varlimsup_{\alpha}\left\|\bar{u}_{\alpha}\right\|_{W^{1,2}(C)} \leq R .
$$

Hence, by replacing with a subnet, for each $C,\left\{\bar{u}_{\alpha}\right\}_{\alpha \in \mathcal{A}} W^{1,2}$-weakly and $L^{2}$ strongly converges to some $u_{C} \in W^{1,2}(C)$ with $\left\|u_{C}\right\|_{W^{1,2}(C)} \leq R$. We take a monotone increasing sequence of $C$ covering $M$ and use the diagonal argument to obtain a function $u \in W^{1,2}(M)$ such that, when replacing with a 
subnet of $\mathcal{A}, \lim _{\alpha} \bar{u}_{\alpha}=\left.u\right|_{C}\left(L^{2}\right.$ strongly $)$ for each $C$, and that $\|u\|_{W^{1,2}} \leq R$. This implies (F1').

To verify (F2), let us take any $u \in W^{1,2}(M)$ and fix it. Since $W^{1,2}(M)=$ $W_{0}^{1,2}(M)$, for any $\epsilon>0$ there is a Lipschitz function $\tilde{u}_{\epsilon}$ in $C_{0}(M)$ such that $\left\|u-\tilde{u}_{\epsilon}\right\|_{W^{1,2}}<\epsilon$. There exists a large $\alpha(\epsilon) \in \mathcal{A}$ such that $C:=\operatorname{supp} \tilde{u}_{\epsilon} \subset$ $f_{\alpha}\left(O_{\alpha}\right)$ for all $\alpha \geq \alpha(\epsilon)$ and that the function $u_{\epsilon} \in W^{1,2}\left(M_{\alpha(\epsilon)}\right)$ defined by $u_{\epsilon}:=\left.\tilde{u}_{\epsilon} \circ f_{\alpha(\epsilon)}\right|_{C}$ on $C$ and by $u_{\epsilon}:=0$ outside $C$ satisfies

$$
\left|\mathcal{E}_{M_{\alpha(\epsilon)}}\left(u_{\epsilon}\right)-\mathcal{E}_{M}\left(\tilde{u}_{\epsilon}\right)\right|<\epsilon \quad \text { and } \quad\left|\left\|u_{\epsilon}\right\|_{L^{2}\left(M_{\alpha(\epsilon)}\right)}-\left\|\tilde{u}_{\epsilon}\right\|_{L^{2}(M)}\right|<\epsilon
$$

Then we have $u_{\epsilon} \rightarrow u$ in $L^{2}(\mathcal{M})$ and $\mathcal{E}_{M_{\alpha(\epsilon)}}\left(u_{\epsilon}\right) \rightarrow \mathcal{E}_{M}(u)$ as $\epsilon \rightarrow 0$. This together with Proposition 2.5 completes the proof.

Remark 3.2. In Theorem 3.1, the equality $\sigma\left(\Delta_{M}\right)=\lim _{\alpha} \sigma\left(\Delta_{M_{\alpha}}\right)$ does not hold in general. In fact, we take a complete noncompact Riemannian manifold $M$ with $\inf \sigma\left(\Delta_{M}\right)>0$ (some hyperbolic manifold satisfy this). Then there exists a net of closed Riemannian manifolds $M_{\alpha}$ which compact Lipschitz converges to $M$. Here, the bottom of spectrum of $\Delta_{M_{\alpha}}$ is $\inf \sigma\left(\Delta_{M_{\alpha}}\right)=0$, so that $\sigma\left(\Delta_{M}\right) \neq \lim _{\alpha} \sigma\left(\Delta_{M_{\alpha}}\right)$.

By looking at Theorem 3.1, it is important to investigate the criteria of the condition $W^{1,2}(M)=W_{0}^{1,2}(M)$. We know the following sufficient conditions for it:

- $M$ is a complete Lipschitz-Riemannian manifold, which is obtained in a standard way (see $[25,26,27]))$.

- $M$ is isometric to $N \backslash S$, where $N$ is a complete smooth Riemannian manifold and $S \subset N$ a compact smooth submanifold of codimension $\geq 2([39,41])$.

- The completion $\bar{M}$ of $M$ is an Alexandrov space of curvature bounded below and $\bar{M} \backslash M$ consists of non-boundary singular points of $\bar{M}$ ([38]).

- $M$ is a Lipschitz-Riemannian manifold and $\bar{M} \backslash M$ has Minkowski codimension $>2$, which is obtained by the same way as in [41].

Let us see some examples of compact Lipschitz convergence in the following: 
3.2.1. Blowing up. Let $M$ be a smooth manifold and $S \subset M$ a closed subset. When a net $\left\{g_{\alpha}\right\}$ of Riemannian metrics on $M$ converges to a complete Riemannian metric $g$ on $M \backslash S$ uniformly on compact sets on $M \backslash S$, it is easy to show that $\left(M, g_{\alpha}\right)$ compact Lipschitz converges to $(M \backslash S, g)$. Since $(M \backslash S, g)$ is complete and by Theorem 3.1, the spectral structure $\Sigma\left(M, g_{\alpha}\right)$ strongly converges to $\Sigma(M \backslash S, g)$.

3.2.2. Magnifying. Let $M$ be a smooth Riemannian manifold with metric $g$, and $r M$ denote the manifold $M$ with metric $r^{2} g, r>0$. Then, as $r \rightarrow \infty$, $r M$ compact Lipschitz converges to $\mathbb{R}^{n}$, where $n:=\operatorname{dim} M$. Therefore, $\Sigma(r M)$ strongly converges to $\Sigma\left(\mathbb{R}^{n}\right)$.

Corollary 3.1. Let $M$ be a smooth Riemannian manifold. Then we have

$$
\lim _{a, b \rightarrow \infty} \frac{a}{b}=1
$$

where $(a, b) \subset[0, \infty)$ is any spectral gap, i.e., $(a, b) \cap \sigma\left(\Delta_{M}\right)=\emptyset$.

Proof. Theorem 3.1 implies that $\sigma\left(\Delta_{r M}\right)=r^{-2} \sigma\left(\Delta_{M}\right)$ converges to $\sigma\left(\mathbb{R}^{n}\right)=$ $[0, \infty)$. This proves the corollary.

3.2.3. Tower of coverings. Let $M_{0} \leftarrow M_{1} \leftarrow M_{2} \leftarrow \ldots$ be a sequence of Riemannian coverings of complete Riemannian manifolds with fundamental groups $G_{k}:=\pi_{1}\left(M_{k}\right), k=0,1,2, \ldots$ Then $G_{k+1}$ is a subgroup of $G_{k}$ for any $k \geq 0$. Since $G_{\infty}:=\bigcap_{k} G_{k}$ is a subgroup of $G_{0}$, there exists a Riemannian covering space $M_{\infty}$ of $M_{0}$ with fundamental group $G_{\infty}$. It is easy to see that $M_{k}$ compact Lipschitz converges to $M_{\infty}$ as $k \rightarrow \infty$ and therefore $\Sigma\left(M_{k}\right)$ strongly converges to $\Sigma\left(M_{\infty}\right)$.

3.2.4. Degeneration. Let $M$ be a smooth manifold and $g$ a degenerate Riemannian metric on $M$, i.e., a positive semi-definite symmetric smooth $(0,2)$-tensor. Then, $g$ induces a pseudo-distance function $d_{g}$ on $M$ and the quotient space of $M$ modulo the equivalence relation $d_{g}(\cdot, \cdot)=0$ becomes an intrinsic metric (or length) space, say $\hat{M}_{g}$. Here, an intrinsic metric space is a metric space $X$ such that the distance between any two points in $X$ is equal to the infimum of length of continuous curves joining them (cf. [28]). Denote by $\pi_{g}: M \rightarrow \hat{M}_{g}$ the projection. The nondegenerate part, say $M_{g}$, of $g$ in $M$ is an open subset of $M$ and the degenerate part, say $S_{g}$, of $g$ in $M$ is closed. We equip $M_{g}$ with the metric $g$, so that $M_{g}$ is isometrically embedded into 
$\hat{M}_{g}$. Assume that a net $\left\{g_{\alpha}\right\}$ of Riemannian metrics converges to $g$ uniformly on compact sets on $M$, in which case we say that $g_{\alpha}$ degenerates to $g$. It is easy to prove that $\left(M, p, g_{\alpha}\right) \mathrm{GH}$ converges to $\left(\hat{M}_{g}, \pi_{g}(p)\right)$ and $\left(M, g_{\alpha}\right)$ compact Lipschitz converges to $M_{g}$.

Example 3.2. Let $M_{\epsilon}:=\left\{(x, y, z) \in \mathbb{R}^{3} \mid x^{2}+y^{2}-z^{2}=\epsilon\right\}$ for $\epsilon \geq 0$. Then, as $\epsilon \rightarrow 0$, the surface $M_{\epsilon}$ compact Lipschitz converges to the double of cone, $C:=M_{0} \backslash\{o\}$, with the origin excluded. We see that $W^{1,2}(C)=W_{0}^{1,2}(C)$ and therefore $\Sigma\left(M_{\epsilon}\right)$ strongly converges to $\Sigma(C)$.

Remark 3.3. Consider the set $\mathcal{R}(c)$ of isometry classes of pointed complete two-dimensional Riemannian manifolds with $L^{1}$ norm of curvature $\leq c$ for a constant $c>0$. In [50], the second author proved the GH relative compactness of $\mathcal{R}(c)$ and characterized the topology of the GH limit spaces of $\mathcal{R}(c)$. Note here that the limit may degenerate partially. It seems not so hard to construct a Lipschitz-Riemannian metric on the nondegenerate part of a limit space and to prove that if a net $M_{\alpha} \in \mathcal{R}(c) \mathrm{GH}$ converges to a space $M$, then the spectral structure $\Sigma\left(M_{\alpha}\right)$ strongly converges to $\Sigma(M)$.

We see some interesting works in $[2,35]$ for degeneration.

\subsection{Convergence of manifolds under a bound of local isoperimetric constant.}

Let $M$ be an $n$-dimensional smooth Riemannian manifold (with boundary). Define the isoperimetric constant of $M$ by

$$
\mathcal{I}(M):=\inf \frac{\operatorname{area}\left(\partial \Omega_{1} \cap \partial \Omega_{2}\right)}{\operatorname{vol}\left(\Omega_{1}\right) \wedge \operatorname{vol}\left(\Omega_{2}\right)}
$$

where $\left(\Omega_{1}, \Omega_{2}\right)$ run over all pairs of two disjoint open domains in $M$ with piecewise smooth boundary such that $\operatorname{vol}\left(M \backslash\left(\Omega_{1} \cup \Omega_{2}\right)\right)=0$, and $x \wedge y:=$ $\min \{x, y\}$ for $x, y \in \mathbb{R}$. For $r>0$ we define the $r$-isoperimetric constant $\mathcal{I}_{r}(M)$ of $M$ by

$$
\mathcal{I}_{r}(M):=r \cdot \sup _{\left\{p_{k}\right\}} \inf _{k} \mathcal{I}\left(B\left(p_{k}, r\right)\right)
$$

where $\left\{p_{k}\right\}$ is any $r$-discrete $r$-net of $M$. Note that $\mathcal{I}_{a r}(a M)=\mathcal{I}_{r}(M)$ for any $a, r>0$. For the completion $\bar{M}$ of an incomplete Riemannian manifold $M$, let us define a measure $\operatorname{vol}_{\bar{M}}$ on $\bar{M}$ to be $\operatorname{vol}_{M}$ on $M$ and zero on $\bar{M} \backslash M$.

Applying Theorem 3.1 and a result in $\S 5$ below, we have the following: 
Theorem 3.2. Let $\left\{M_{\alpha}\right\}_{\alpha \in \mathcal{A}}$ be a net of compact Riemannian manifold (possibly with boundary) and $M$ an incomplete Riemannian manifold. We assume the following (1)-(5).

(1) $\varlimsup_{\lim _{r} \backslash 0} \underline{\lim }_{\alpha} \mathcal{I}_{r}\left(M_{\alpha}\right)>0$.

(2) $W^{1,2}(M)=W_{0}^{1,2}(M)$.

(3) The completion $\bar{M}$ of $M$ is compact.

(4) $\left(M_{\alpha}, \operatorname{vol}_{M_{\alpha}}\right)$ measured $G H$ converges to $\left(\bar{M}, \operatorname{vol}_{\bar{M}}\right)$.

(5) $M_{\alpha}$ compact Lipschitz converges to $M$.

Then, the spectral structure $\Sigma\left(M_{\alpha}\right)$ compactly converges to $\Sigma(M)$. In particular, $\Sigma(M)$ has compact resolvent and for any fixed $k \in \mathbb{N}$, the $k^{\text {th }}$ eigenvalue of $\Delta_{M_{\alpha}}$ converges to that of $\Delta_{M}$.

Proof. Consider the family $\mathcal{S}_{c}\left(L^{2}\left(\mathcal{M}_{c}\right)\right)$ of spectral structures on $L^{2}(X ; m)$ with compact resolvent, where $(X, p, m) \in \mathcal{M}_{c}$ runs over all spaces. Recall that $\mathcal{S}_{c}\left(L^{2}\left(\mathcal{M}_{c}\right)\right)$ has the compact spectral topology based on the measured GH topology on $\mathcal{M}_{c}$. Suppose that $\Sigma\left(M_{\alpha}\right)$ does not compactly converge to $\Sigma(M)$, so that there exist a subnet $\mathcal{B}$ of $\mathcal{A}$ and a neighborhood $U$ in $\mathcal{S}_{c}\left(L^{2}\left(\mathcal{M}_{c}\right)\right)$ of $\Sigma(M)$ such that $\Sigma\left(M_{\beta}\right)$ for any $\beta \in \mathcal{B}$ is not contained in $U$. According to Corollary 1 of [57], the assumption for isoperimetric constant, (1), implies the estimate for the Poincaré constant, (P) in $§ 5$. Also, (D) and $(\mathrm{N})$ in $\S 5$ are clear. Hence, Theorem 5.1 below shows the existence of a compactly convergent subnet of $\left\{\Sigma\left(M_{\beta}\right)\right\}$. By Theorem 3.1, the limit must be $\Sigma(M)$, which is a contradiction. Thus, $\Sigma\left(M_{\alpha}\right)$ compactly converges to $\Sigma(M)$.

The rest follows from Remark 2.6 and Corollary 2.5. This completes the proof.

Remark 3.4. (1) In general, a net of manifolds $\left\{M_{\alpha}\right\}$ in Theorem 3.2 has no bound of the isoperimetric constant of Chavel-Feldman [12]. We shall see such an example in Example 3.3 below. It is not clear that there exists a bound of heat kernel needed to apply the theory of Kasue-Kumura [32], for manifolds satisfying (1)-(5) of the theorem. On the other hand, if the heat kernel is bounded in the sense of [33], or if the isoperimetric constant is bounded in the sense of [12], then this does not necessarily imply (1) of Theorem 3.2. 
(2) Both of the assumptions (4) and (5) of Theorem 3.2 can be replaced with the following equivalent condition: There exists bi-Lipschitz homeomorphisms $f_{\alpha}$ from open subsets $O_{\alpha} \subset M_{\alpha}$ to relatively compact open subsets $O_{\alpha}^{\prime} \subset M$ such that $\left\{O_{\alpha}^{\prime}\right\}$ is a monotone increasing net covering $M$ and that

$$
\lim _{\alpha} \operatorname{vol}\left(M_{\alpha} \backslash O_{\alpha}\right)=\lim _{\alpha} \sup _{x \in M_{\alpha} \backslash O_{\alpha}} d\left(x, O_{\alpha}\right)=0
$$

(3) Theorem 3.2 is true also for Lipschitz-Riemannian manifolds instead of smooth Riemannian manifolds, which follows from the same proof.

We see more definite cases of Theorem 3.2 in the following sections.

3.3.1. Shrinking. Let $M$ be a smooth Riemannian manifold with $W^{1,2}(M)=W_{0}^{1,2}(M)$, and $N \subset M$ a compact smooth submanifold of codimension $\geq 2$. It then follows that $W_{0}^{1,2}(M \backslash N)=W_{0}^{1,2}(M)$ and so $\Sigma(M \backslash N)=\Sigma(M)$. We take a family $\left\{M_{r}\right\}_{r \in\left(0, r_{0}\right)}$ of smooth Riemannian manifolds in such a way that each $M_{r}$ contains an open subset $U_{r}$ such that $M_{r} \backslash U_{r}$ is isometric to $M \backslash B(N, r)$, where $B(N, r)$ is the open $r$-ball of $N$. Then, as $r \rightarrow 0, M_{r}$ compact Lipschitz converges to $M \backslash N$. Thus, by Theorem 3.1, the spectral structure $\Sigma\left(M_{r}\right)$ strongly converges to $\Sigma(M)$ as $r \rightarrow 0$.

Assume further that $M$ is compact, $N$ consists of a single point, the diameter and volume of $U_{r}$ both tend to zero as $r \rightarrow 0$, and $r \cdot \mathcal{I}\left(U_{r}\right)$ is bounded away from zero. Then, it is easy to verify that all the assumptions of Theorem 3.2 are satisfied, so that the spectral structure $\Sigma\left(M_{r}\right)$ compactly converges to $\Sigma(M)$.

A nontrivial example of such a family $\left\{U_{r}\right\}$ is seen in the following:

Example 3.3. Let $T:=(\mathbb{R} / 2 \pi \mathbb{Z}) \times S$ with coordinate $(\theta, s)$ for a compact manifold $S$ with a Riemannian metric $d s^{2}$, and let $\left\{f_{r}: \mathbb{R} / 2 \pi \mathbb{Z} \rightarrow\right.$ $(0, \infty)\}_{r \in\left(0, r_{0}\right)}$ be a family of smooth functions such that

(1) $f_{r}$ for each $r$ is monotone nonincreasing on $[0, \pi]$ and monotone nondecreasing on $[\pi, 2 \pi]$,

(2) we have $\lim _{r \searrow 0} f_{r}(\pi)=0$,

(3) there is an $a \in(0, \pi)$ such that $\left.f_{r}\right|_{[-a, a]} \equiv 1$ for any $r$. 
(For instance, we may take $f_{r}(\theta)$ as a smooth deformation of $(\cos \theta+1+r) \wedge 1$.) Assuming that $\left(S, d s^{2}\right)$ contains a flat disk of radius $a \in(0, \pi)$, say $B(x, a)$, we find a flat disk of radius $a, B((0, x), a)$, in the Riemannian manifold $\left(T, d \theta^{2}+f_{r}(\theta)^{2} d s^{2}\right)$ by (3). We consider the rescaled Riemannian manifold $T_{r}:=\left(T,(r / a)^{2}\left(d \theta^{2}+f_{r}(\theta)^{2} d s^{2}\right)\right)$ and so the flat disk is rescaled as one with radius $r$. Remove the disk from $T_{r}$ and denote the rest by $U_{r}$. We deform the metric of $U_{r}$ around $\partial U_{r}$ such that the gluing $M_{r}$ of $M \backslash B(N, r)$ and $U_{r}$ along their boundaries is a smooth Riemannian manifold. Then, the construction of $U_{r}$ yields that the diameter and volume of $U_{r}$ both tend to zero as $r \rightarrow 0$ and that $\inf _{r} r \cdot \mathcal{I}\left(U_{r}\right)>0$.

For such a family $\left\{M_{r}\right\}$, we now consider the condition that

$$
\inf _{r>0, \Omega \subset M_{r}} \frac{\operatorname{area}(\partial \Omega)^{n}}{\operatorname{vol}(\Omega)^{n-1}}>0
$$

where $n:=\operatorname{dim} M_{r}$. Note that this is required in [12] to obtain convergence of eigenvalue. Let us see that by choosing a suitable $f_{r}$, the family $\left\{M_{r}\right\}$ does not satisfy (3.4). In fact, it follows that (3.4) does not hold if

$$
\lim _{r \rightarrow 0} \inf _{a<\theta_{1}<\theta_{2}<2 \pi-a} \frac{\left(f_{r}\left(\theta_{1}\right)+f_{r}\left(\theta_{2}\right)\right)^{n}}{\left(\int_{\theta_{1}}^{\theta_{2}} f_{r}(\theta) d \theta\right)^{n-1}}=0,
$$

(cf. $\S 8$ of [33]). For instance, if $f_{r}(\theta)$ tends to $|\theta-\pi|^{n} \wedge 1$ as $r \rightarrow 0$, we have $(3.5)$.

3.3.2. Iteration of attaching small manifolds. For a fixed $n \geq 2$, let $\left\{N_{k}\right\}_{k=0,1,2, \ldots}$ be a sequence of $n$-dimensional closed Riemannian manifolds such that $\operatorname{vol}\left(N_{k}\right), \operatorname{diam}\left(N_{k}\right)$, and $1 / \mathcal{I}\left(N_{k}\right)$ are all uniformly bounded from above. (For example, such the boundedness is obtained if the Ricci curvature of $N_{k}$ satisfies $\operatorname{Ric}_{N_{k}} \geq n-1$.) Assume that every $N_{k}$ contains two disjoint unit open metric balls, say $B\left(p_{k}, 1\right)$ and $B\left(q_{k}, 1\right)$, both isometric to $B^{n}(1)$, where $B^{n}(r)$ denotes an $n$-dimensional Euclidean open disk of radius $r$. We shall construct another sequence of closed Riemannian manifolds $\left\{M_{k}\right\}$ such that each $M_{k}$ contains a metric ball $B\left(x_{k}, 100^{-k}\right)$ isometric to $B^{n}\left(100^{-k}\right)$. We first set $M_{0}:=N_{0}$ and $x_{0}:=q_{0}$. Supposing that $M_{k}$ is defined for a number $k$, we define $M_{k+1}$ as the connected sum of $M_{k}$ and $N_{k}^{\prime}:=100^{-(k+1)} N_{k}$ with the metric described as follows. We deform the metrics of $\hat{M}_{k}:=M_{k} \backslash B\left(x_{k}, 100^{-(k+1)} / 2\right)$ and $\hat{N}_{k}:=N_{k}^{\prime} \backslash B\left(p_{k+1}, 100^{-(k+1)} / 2\right)$ around the boundaries so that the gluing of $\hat{M}_{k}$ and $\hat{N}_{k}$ along their boundaries is a smooth Riemannian manifold, which is the desired $M_{k+1}$. A point 
$x_{k+1} \in M_{k+1}$ is defined to be $q_{k+1}$ in $\hat{N}_{k}$. Then, $B\left(x_{k+1}, 100^{-(k+1)}\right)$ is isometric to $B^{n}\left(100^{-(k+1)}\right)$.

By the construction, we have the infinite sequence of the isometric embeddings

$$
M_{0} \backslash B\left(x_{0}, 1\right) \hookrightarrow M_{1} \backslash B\left(x_{1}, 100^{-1}\right) \hookrightarrow \ldots \hookrightarrow M_{k} \backslash B\left(x_{k}, 100^{-k}\right) \hookrightarrow \ldots
$$

The inductive limit, say $M$, is a precompact incomplete Riemannian manifold with finite volume such that $\bar{M} \backslash M$ consists of a single point. Remark that if we take each $N_{k}$ to be non-simply connected, then $\pi_{1}(M)$ is not finitely generated. It is easy to verify that $\left\{M_{k}\right\}$ and $M$ together satisfy the assumptions (1)-(5) of Theorem 3.2. Here, (1) is deduced from $\sup _{k} \mathcal{I}\left(N_{k}\right)<\infty$, and (2) is proved by constructing cut-off functions around $\bar{M} \backslash M$ in a similar way as in $[10,39]$, for which we omit the detail. Thus, $\Sigma\left(M_{k}\right)$ compactly converges to $\Sigma(M)$.

\subsection{Collapsing of warped product manifolds.}

Let $\left\{N_{\alpha}\right\}_{\alpha \in \mathcal{A}}$ be a net of (incomplete) Riemannian manifolds which compact Lipschitz converges to a Riemannian manifold $N$, let $F$ be a compact Riemannian manifold, and let $\left\{\varphi_{\alpha}: N_{\alpha} \rightarrow(0, \infty)\right\}_{\alpha \in \mathcal{A}}$ be a net of $C^{\infty}$ functions. We consider the warped product $M_{\alpha}:=N_{\alpha} \times_{\varphi_{\alpha}} F$, i.e., $M_{\alpha}$ is a product manifold $N_{\alpha} \times F$ with Riemannian metric $g_{M_{\alpha}}:=g_{N_{\alpha}}+\varphi_{\alpha}^{2} g_{F}$, where $g_{X}$ denotes the metric of a Riemannian manifold $X$. We denote the projection by $\pi_{N_{\alpha}}: M_{\alpha} \rightarrow N_{\alpha}$. Assume that there exist a net $r_{\alpha} \rightarrow 0$ of positive numbers and a constant $c \in(0,1)$ such that for any $\alpha \in \mathcal{A}$ and any $x \in N_{\alpha}$,

$$
c \leq \frac{\varphi_{\alpha}(x)}{r_{\alpha}} \leq c^{-1} .
$$

Then, by taking a subnet of $\mathcal{A}$, the push-forward $\left(\pi_{N_{\alpha}}\right)_{*} \mu_{\alpha}$ of the measure $\mu_{\alpha}:=r_{\alpha}^{-k} \operatorname{vol}_{M_{\alpha}}$ vaguely converges to a positive Radon measure $m$ on $N$, where $k:=\operatorname{dim} F$. Since

$$
\mu_{\alpha}(d x d y)=r_{\alpha}^{-k} \varphi_{\alpha}^{k}(x) \operatorname{vol}_{F}(d y) \operatorname{vol}_{N_{\alpha}}(d x),
$$

the measures $m$ and $\operatorname{vol}_{N}$ are mutually absolute continuous.

Theorem 3.3. Assume that $W^{1,2}(N ; m)=W_{0}^{1,2}(N ; m)$. Then, the spectral structure $\Sigma\left(M_{\alpha}, \mu_{\alpha}\right)$ strongly converges to $\Sigma(N, m)$. If the embedding of $W^{1,2}(N ; m)$ into $L^{2}(N ; m)$ is compact and if $N_{\alpha}$ Lipschitz converges to $N$, then $\Sigma\left(M_{\alpha}, \mu_{\alpha}\right)$ compactly converges to $\Sigma(N, m)$. 
Proof. Let us prove the later assertion of the theorem. Let $u \in L^{2}\left(M_{\alpha} ; \mu_{\alpha}\right)$ be any function. We set

$$
\bar{u}(x):=\frac{1}{\operatorname{vol}(F)} \int_{F} u(x, y) \operatorname{vol}_{F}(d y), \quad x \in N_{\alpha} .
$$

Assume that $u$ is of $C^{\infty}$. Since $\left|d_{(x, y)} u(x, y)\right|_{M_{\alpha}}^{2}=\left|d_{x} u(x, y)\right|_{N_{\alpha}}^{2}+$ $\varphi_{\alpha}^{-2}(x)\left|d_{y} u(x, y)\right|_{F}^{2}$ for any $x \in N_{\alpha}$ and $y \in F$, we have

$$
\mathcal{E}_{\left(N_{\alpha},\left(\pi_{N_{\alpha}}\right)_{*} \mu_{\alpha}\right)}(\bar{u}) \leq \mathcal{E}_{\left(M_{\alpha} ; \mu_{\alpha}\right)}(u) .
$$

It follows from the Poincaré inequality on $F$ that

$$
\begin{aligned}
\int_{F}(u(x, y)-\bar{u}(x))^{2} \operatorname{vol}_{F}(d y) & \leq \operatorname{const} \int_{F}\left|d_{y} u(x, y)\right|_{F}^{2} \operatorname{vol}_{F}(d y) \\
& \leq \operatorname{const} r_{\alpha}^{2} \int_{F}\left|d_{(x, y)} u(x, y)\right|_{F}^{2} \operatorname{vol}_{F}(d y)
\end{aligned}
$$

where 'const' is a constant depending on $F$. Hence,

$$
\begin{aligned}
& \left\|u-\bar{u} \circ \pi_{N_{\alpha}}\right\|_{L^{2}\left(M_{\alpha} ; \mu_{\alpha}\right)}^{2} \\
& \leq \operatorname{const} r_{\alpha}^{2} \int_{N_{\alpha} \times F} \int_{F}\left|d_{(x, y)} u(x, y)\right|_{F}^{2} \operatorname{vol}_{F}(d y) r^{-k} \varphi^{k}(x) \operatorname{vol}_{F}\left(d y_{1}\right) \operatorname{vol}_{N_{\alpha}}(d x) \\
& \leq \operatorname{const} r_{\alpha}^{2} \mathcal{E}_{\left(M_{\alpha}, \mu_{\alpha}\right)}(u)
\end{aligned}
$$

Now, let $u_{\alpha} \in W^{1,2}\left(M_{\alpha} ; \mu_{\alpha}\right)$ be a net with

$$
\sup _{\alpha}\left(\left\|u_{\alpha}\right\|_{L^{2}\left(M_{\alpha} ; \mu_{\alpha}\right)}^{2}+\mathcal{E}_{\left(M_{\alpha}, \mu_{\alpha}\right)}\left(u_{\alpha}\right)\right)<\infty
$$

There exists a net of $\epsilon_{\alpha}$-almost isometries $f_{\alpha}: N_{\alpha} \rightarrow N, \epsilon_{\alpha} \rightarrow 0$. By (3.7) and (3.8), we have

$$
\sup _{\alpha}\left(\left\|\bar{u}_{\alpha} \circ f_{\alpha}^{-1}\right\|_{L^{2}(N ; m)}^{2}+\mathcal{E}_{(N, m)}\left(\bar{u}_{\alpha} \circ f_{\alpha}^{-1}\right)\right)<\infty
$$

which implies the existence of an $L^{2}(N ; m)$-strongly convergent subnet of $\left\{\bar{u}_{\alpha} \circ f_{\alpha}^{-1}\right\}$. Therefore, $\left\{\mathcal{E}_{\left(M_{\alpha}, \mu_{\alpha}\right)}\right\}$ is asymptotically compact. Assume that $\left\{\bar{u}_{\alpha} \circ f_{\alpha}^{-1}\right\}$ converges to a function $u \in L^{2}(N ; m)$ strongly in $L^{2}(N ; m)$. Then, by $(3.7)$,

$$
\mathcal{E}_{(N, m)}(u) \leq \underline{\lim } \mathcal{E}_{\left(N_{\alpha},\left(\pi_{N_{\alpha}}\right) * \mu_{\alpha}\right)}\left(\bar{u}_{\alpha}\right) \leq \underline{\lim } \mathcal{E}_{\left(M_{\alpha} ; \mu_{\alpha}\right)}\left(u_{\alpha}\right),
$$

which proves (F1). 
For any fixed $u \in W^{1,2}(N ; m)$, we set $u_{\alpha}:=u \circ f_{\alpha} \circ \pi_{N_{\alpha}}$. Then, $u_{\alpha} \rightarrow u$ in $L^{2}(\mathcal{M})$ and $\mathcal{E}_{\left(M_{\alpha}, \mu_{\alpha}\right)}\left(u_{\alpha}\right) \rightarrow \mathcal{E}_{(N, m)}(u)$. This proves (F2).

The proof of the first assertion is similar to that of Theorem 3.1 and is omitted.

Note that it is difficult to relax the assumption (3.6). See $[1,35]$ for such attempts.

\subsection{Convergence of noncompact Alexandrov spaces.}

Denote by $\mathcal{A}(n, D, v)$ the set of compact $n$-dimensional Alexandrov spaces of curvature $\geq-1$, diameter $\leq D$, and $n$-dimensional Hausdorff measure $\geq v$, where $n \in \mathbb{N}, D, v>0$ are constants. In [51], we proved that the topology induced from the spectral distance coincides with the GH topology on $\mathcal{A}(n, D, v)$. In particular, if a net $\left\{M_{\alpha}\right\} \subset \mathcal{A}(n, D, v) \mathrm{GH}$ converges to an $M \in \mathcal{A}(n, D, v)$, then the spectral structure $\Sigma\left(M_{\alpha}\right)$ compactly converges to $\Sigma(M)$. Here, we equip $n$-dimensional Alexandrov spaces with the $n$ dimensional Hausdorff measure $\mathcal{H}^{n}$. The purpose of this section is to prove the following:

Theorem 3.4. Let $\left\{\left(M_{\alpha}, p_{\alpha}\right)\right\}$ be a net of (possibly noncompact) pointed $n$-dimensional Alexandrov spaces of curvature $\geq-1$ which $G H$ converges to a pointed $n$-dimensional Alexandrov space $(M, p)$. Then, $\left(M_{\alpha}, p_{\alpha}, \mathcal{H}^{n}\right)$ converges to $\left(M, p, \mathcal{H}^{n}\right)$ with respect to the measured $G H$ topology and the spectral structure $\Sigma\left(M_{\alpha}\right)$ strongly converges to $\Sigma(M)$.

As a consequence to the theorem, we prove:

Corollary 3.2. Let $M$ be a noncompact nonnegatively curved Alexandrov space such that $\lim _{r \rightarrow \infty} \mathcal{H}^{n}(B(p, r)) / r^{n}>0$ for some point $p \in M$, where $n:=\operatorname{dim} M$. Then, we have inf $\sigma\left(\Delta_{M}\right) \backslash\{0\}=0$, and spectral gaps $(a, b) \subset$ $[0, \infty) \backslash \sigma\left(\Delta_{M}\right)$ satisfy

$$
\lim _{a, b \rightarrow 0} \frac{a}{b}=1
$$

We briefly describe some convention for Alexandrov spaces. Refer to $[8,45,38]$ for the details. Let $M$ be an $n$-dimensional Alexandrov space with curvature $\geq-1$. For $\delta>0$, the $\delta$-singular set $S_{\delta}$ of $M$ is defined to be the set of $x \in M$ such that $\mathcal{H}^{n-1}\left(\Sigma_{x}\right) \leq \omega_{n-1}-\delta$, where $\Sigma_{x}$ is the space of directions at $x$ and $\omega_{n-1}$ the volume of the unit $(n-1)$-sphere. Then, the $\delta$-singular set for any $\delta>0$ is of Hausdorff dimension $\leq n-1([8,45])$. 
There exists a $\delta_{n}>0$ depending only on the dimension $n$ such that $M \backslash S_{\delta_{n}}$ is a (incomplete) Lipschitz-Riemannian manifold ([45]). The canonical spectral structure $\Sigma(M)$ on $L^{2}\left(M ; \mathcal{H}^{n}\right)$ is induced from the canonical Dirichlet form $\left(\mathcal{E}_{M}, W_{0}^{1,2}\left(M \backslash S_{\delta_{n}}\right)\right)$ as defined in $\S 3.1$. The double $\operatorname{dbl}(M)$ of $M$ is obtained by gluing two copies of $M$ along their boundaries. The double of an Alexandrov space with nonempty boundary is an Alexandrov space without boundary and of the same lower bound of curvature $([8,46])$. We have a natural isometric embedding $\iota: M \rightarrow \operatorname{dbl}(M)$. Denote by $\hat{S}_{\delta}$ the preimage in $M$ of the $\delta$-singular set of $\operatorname{dbl}(M)$ contained in $\iota(M)$. Clearly, if $\partial M=\emptyset$, then $\hat{S}_{\delta}=S_{\delta}$. It follows that $\hat{S}_{\delta} \subset S_{\delta}$ and $S_{\delta} \backslash \hat{S}_{\delta} \subset \partial M$. The two following theorems are essential for the proof of Theorem 3.4.

Theorem 3.5 (Theorem 1.1 of [38]). The set $\hat{S}_{\delta}$ for any $\delta>0$ is of capacity zero, i.e., $W_{0}^{1,2}\left(M \backslash \hat{S}_{\delta}\right)=W^{1,2}\left(M \backslash \hat{S}_{\delta}\right)$.

Theorem 3.6 (Theorem 3.1 of [51]). Assume that a net $\left\{\left(M_{\alpha}, p_{\alpha}\right)\right\}$ of pointed $n$-dimensional Alexandrov spaces of curvature $\geq-1 G H$ converges to a pointed $n$-dimensional Alexandrov space $(M, p)$. Then, for any $\delta$ with $0<\delta \ll 1 / n$, there exist $\epsilon_{\alpha}$-approximations $f_{\delta, \alpha}:(M, p) \rightarrow\left(M_{\alpha}, p_{\alpha}\right)$ for some $\epsilon_{\alpha} \searrow 0$ and compact subsets $D_{\delta, \alpha} \subset M \backslash \hat{S}_{\delta}$ with $\bigcup_{\alpha} D_{\delta, \alpha}=M \backslash \hat{S}_{\delta}$ such that the restriction $f_{\delta, \alpha}: D_{\delta, \alpha} \rightarrow f_{\delta, \alpha}\left(D_{\delta, \alpha}\right)$ is a $\theta(\delta)$-almost isometry, and

$$
\lim _{\alpha} \mathcal{H}^{n}\left(M_{\alpha} \backslash f_{\delta, \alpha}\left(D_{\delta, \alpha}\right)\right)=0,
$$

where $\theta$ is some function with $\lim _{t \rightarrow 0} \theta(t)=0$. In particular, $\left(M_{\alpha}, p_{\alpha}, \mathcal{H}^{n}\right)$ converges to $\left(M, p, \mathcal{H}^{n}\right)$ in the measured $G H$ topology.

Indeed, we assumed the compactness of Alexandrov spaces in the original statement in [51], which is however not essential for the proof.

Proof of Theorem 3.4. The theorem is proved in the same way as in the proof of Theorem 3.1 by using the above two theorems. See also the discussion in $\S 5$ of $[51]$.

Proof of Corollary 3.2. We first claim that the Laplacian of an Alexandrov space which is isometric to an Euclidean cone has full spectrum $[0, \infty)$. In fact, this follows from the invariability of the spectrum up to metric rescaling and the non-triviality of the Laplacian.

Let $M$ be a noncompact nonnegatively curved Alexandrov space. Consider the $\epsilon$-rescaled space $\epsilon M, \epsilon>0$. For a fixed point $p \in M$, as 
$\epsilon \rightarrow 0,(\epsilon M, p) \mathrm{GH}$ converges to an Euclidean cone, called the limit cone ' $C_{\infty} M$ ' of $M$ (see $\S 4$ of [6] and Proposition 1.1 of [49]). The assumption $\lim _{r \rightarrow \infty} \mathcal{H}^{n}(B(p, r)) / r^{n}>0$ implies that this convergence does not collapse dimension. Applying Theorem 3.4 and Proposition 2.5 yields that $\epsilon^{-2} \sigma\left(\Delta_{M}\right)=\sigma\left(\Delta_{\epsilon M}\right) \rightarrow \sigma\left(\Delta_{C_{\infty} M}\right)=[0, \infty)$ as $\epsilon \rightarrow 0$. This completes the proof.

\section{Convergence of graphs.}

\subsection{Graph with simplicial metric.}

A (oriented) graph $\Gamma$ is defined to be a set $\left(V_{\Gamma}, E_{\Gamma}, o, t\right)$, where $V_{\Gamma}$ and $E_{\Gamma}$ are at most countable sets and $o, t: E_{\Gamma} \rightarrow V_{\Gamma}$ are maps. We always assume that all graphs are locally finite, i.e., for any $x \in o\left(E_{\Gamma}\right)$ and $y \in t\left(E_{\Gamma}\right), o^{-1}(x)$ and $t^{-1}(y)$ are both finite subsets of $E_{\Gamma}$. Each element of $V_{\Gamma}$ is called a vertex of $\Gamma$ and each element of $E_{\Gamma}$ an edge of $\Gamma$. We say that an edge e $\in E_{\Gamma}$ connects a vertex $x \in V_{\Gamma}$ to a vertex $y \in V_{\Gamma}$ if $o(e)=x$ and $t(e)=y$. Note that for two vertices $x, y \in V_{\Gamma}$, an edge connecting $x$ to $y$ (if any) is not necessarily unique and that we may have a loop, i.e., an edge $e \in E_{\Gamma}$ with $o(e)=t(e)$. We denote the disjoint union $V_{\Gamma} \sqcup E_{\Gamma}$ for a graph $\Gamma$ by the same symbol $\Gamma$.

A simplicial metric on a graph $\Gamma$ is defined to be a pair of two functions $\ell, w: E_{\Gamma} \rightarrow(0, \infty)$. Here, $\ell$ is called the length function and $w$ the weight function. Let $(\Gamma, g)$ be a given graph with a simplicial metric $g=(\ell, w)$. Denote by $C(S)$ the set of real valued functions on a subset $S \subset \Gamma=V_{\Gamma} \sqcup E_{\Gamma}$. The difference operator $d: C\left(V_{\Gamma}\right) \rightarrow C\left(E_{\Gamma}\right)$ is defined by

$$
d u(e):=u(t(e))-u(o(e)), \quad u \in C\left(V_{\Gamma}\right), e \in E_{\Gamma} .
$$

Define a measure $m_{g}$ over $\Gamma$ induced from the metric $g$ by

$$
m_{g}:=\sum_{x \in V_{\Gamma}} w(x) \delta_{x}+\sum_{e \in E_{\Gamma}} \ell(e) \delta_{e}
$$

where $\delta$. denotes the Dirac delta measure. This defines the $L^{2}$ space $L^{2}\left(V_{\Gamma}\right):=L^{2}\left(V_{\Gamma}, \mathbb{R} ;\left.m_{g}\right|_{V_{\Gamma}}\right)$ in the usual manner. For $\omega_{1}, \omega_{2} \in C\left(E_{\Gamma}\right)$ we set the $L^{2}$ inner product:

$$
\left(\omega_{1}, \omega_{2}\right)_{L^{2}}:=\int_{e \in E_{\Gamma}}\left\langle\omega_{1}(e), \omega_{2}(e)\right\rangle_{g} m_{g}(d e)=\sum_{e \in E_{\Gamma}} \frac{\omega_{1}(e) \omega_{2}(e)}{\ell(e)}
$$

if it converges, where $\langle x, y\rangle_{g}:=x y / \ell(e)^{2}$ for $x, y \in \mathbb{R}$. Then, as well as for (Lipschitz-)Riemannian manifold, we obtain the spaces $L^{2}\left(E_{\Gamma}\right):=\{\omega \in$ 
$\left.C\left(E_{\Gamma}\right) \mid\|\omega\|_{L^{2}}:=\sqrt{(\omega, \omega)_{L^{2}}}<\infty\right\}$ and $W^{1,2}\left(V_{\Gamma}\right):=\left\{u \in L^{2}\left(V_{\Gamma}\right) \mid d u \in\right.$ $\left.L^{2}\left(E_{\Gamma}\right)\right\}$. A closed symmetric bilinear form $\mathcal{E}_{(\Gamma, g)}: W^{1,2}\left(V_{\Gamma}\right) \times W^{1,2}\left(V_{\Gamma}\right) \rightarrow$ $\mathbb{R}$ is defined by

$$
\mathcal{E}_{(\Gamma, g)}(u, v):=(d u, d v)_{L^{2}}, \quad u, v \in W^{1,2}\left(V_{\Gamma}\right) .
$$

An inner product $(\cdot, \cdot)_{W^{1,2}}$ on $W^{1,2}\left(V_{\Gamma}\right)$ is defined in the same manner as for (Lipschitz-)Riemannian manifold. Consider the $W^{1,2}$ closure $W_{0}^{1,2}\left(V_{\Gamma}\right)$ of the set, say $C_{0}\left(V_{\Gamma}\right)$, of functions on $V_{\Gamma}$ whose support is a finite subset of $V_{\Gamma}$. We call the restricted closed form $\left(\mathcal{E}_{(\Gamma, g)}, W_{0}^{1,2}\left(V_{\Gamma}\right)\right)$ the canonical Dirichlet form induced from $g$. Note that this is a non-local regular Dirichlet form on $L^{2}\left(V_{\Gamma}\right)$ in the sense of the abstract Dirichlet form theory (see $\S 5.1$ ). The spectral structure $\Sigma(\Gamma, g)$ on $L^{2}\left(V_{\Gamma}\right)$ associated with the simplicial metric $g$ is defined to be that induced from the Dirichlet form $\left(\mathcal{E}_{(\Gamma, g)}, W_{0}^{1,2}\left(V_{\Gamma}\right)\right)$. Define a linear operator $\delta: C\left(E_{\Gamma}\right) \rightarrow C\left(E_{\Gamma}\right)$ by

$$
\delta \omega(x):=\frac{1}{w(x)} \sum_{e \in E_{x}} \frac{\omega(e)}{\ell(e)}, \quad \omega \in C\left(E_{\Gamma}\right), x \in V_{\Gamma},
$$

where $E_{x}$ denotes the set of all edges connecting $x$, and the Laplacian $\tilde{\Delta}=$ $\tilde{\Delta}_{(\Gamma, g)}:=\delta d: C\left(V_{\Gamma}\right) \rightarrow C\left(V_{\Gamma}\right)$. The (formal) adjoint $d^{*}$ of $\left.d\right|_{W^{1,2}\left(V_{\Gamma}\right)}$ satisfies $\left.\delta\right|_{C_{0}\left(V_{\Gamma}\right)} \subset d^{*} \subset \delta$, and the generator, say $\Delta=\Delta_{(\Gamma, g)}$, of $\left(\mathcal{E}_{(\Gamma, g)}, W_{0}^{1,2}\left(V_{\Gamma}\right)\right)$ does $\left.\tilde{\Delta}\right|_{C_{0}\left(V_{\Gamma}\right)} \subset \Delta \subset \tilde{\Delta}$.

\subsection{Convergence of graphs and spectral structure.}

Denote by $\mathcal{G}$ the set of isomorphism classes of pairs $(\Gamma, g)$, where $\Gamma$ runs over all locally finite graphs and $g$ simplicial metrics on $\Gamma$. Let $(\Gamma, g) \in \mathcal{G}$ and denote by $M_{\Gamma}$ the geometric image of $\Gamma$. The length function of $g$ induces a natural distance function, say $d_{g}$, on $M_{\Gamma}$ so that $\left(M_{\Gamma}, d_{g}\right)$ is a locally compact intrinsic metric space. Let $\iota: V_{\Gamma} \rightarrow M_{\Gamma}$ be the natural embedding and define a measure $\mu_{g}$ on $M_{\Gamma}$ to be zero on $M_{\Gamma} \backslash \iota\left(V_{\Gamma}\right)$ and the push-forward measure $\iota_{*}\left(\left.m_{g}\right|_{V_{\Gamma}}\right)$ on $\iota\left(V_{\Gamma}\right)$.

Proposition 4.1. Let $(\Gamma, g) \in \mathcal{G}$ and let $\left\{\left(\Gamma_{\alpha}, g_{\alpha}\right)\right\}_{\alpha \in \mathcal{A}} \subset \mathcal{G}$ be a net such that $\Gamma$ and $\Gamma_{\alpha}$ are all finite graphs. If $\left(M_{\Gamma_{\alpha}}, \mu_{g_{\alpha}}\right)$ converges to $\left(M_{\Gamma}, \mu_{g}\right)$ with respect to the measured $G H$ topology, then the spectral structure $\Sigma\left(\Gamma_{\alpha}, g_{\alpha}\right)$ compactly converges to $\Sigma(\Gamma, g)$ and in particular

$$
\lim _{\alpha} \sigma\left(\Delta_{\left(\Gamma_{\alpha}, g_{\alpha}\right)}\right)=\sigma\left(\Delta_{(\Gamma, g)}\right)
$$


Proof. The proposition is implied by the discussion of $[22,30]$. Let $f_{\alpha}$ : $M_{\Gamma_{\alpha}} \rightarrow M_{\Gamma}$ be measured $\epsilon_{\alpha}$-approximations with respect to $d_{g_{\alpha}}, m_{g}, d_{g}, m_{g}$ for some $\epsilon_{\alpha} \searrow 0$. We may assume that each $f_{\alpha}$ maps vertices to vertices and set $\Phi_{\alpha} u:=\left.u \circ f_{\alpha}\right|_{V_{\Gamma_{\alpha}}} \in C\left(V_{\Gamma_{\alpha}}\right)$ for $u \in C\left(V_{\Gamma}\right)$. It was obtained in [22, 30] that

$$
\mathcal{E}_{(\Gamma, g)}(u)=\lim _{\alpha} \mathcal{E}_{\left(\Gamma_{\alpha}, g_{\alpha}\right)}\left(\Phi_{\alpha} u\right) \quad \text { and } \quad\|u\|_{L^{2}}=\lim _{\alpha}\left\|\Phi_{\alpha} u\right\|_{L^{2}}
$$

for any $u \in C\left(V_{\Gamma}\right)$. This implies (F2). Also, if we define $\Psi_{\alpha}: C\left(V_{\Gamma_{\alpha}}\right) \rightarrow$ $C\left(V_{\Gamma}\right)$ by

$$
\Psi_{\alpha} u(x):=\frac{1}{m\left(f_{\alpha}^{-1}(x)\right)} \int_{f_{\alpha}^{-1}(x) \cap V_{\Gamma}} u d m_{g_{\alpha}}, \quad u \in C\left(V_{\Gamma_{\alpha}}\right), x \in V_{\Gamma},
$$

then

$$
\lim _{\alpha}\left\|\Psi_{\alpha} u_{\alpha}\right\|_{L^{2}}=1, \quad \frac{\lim }{\alpha} \mathcal{E}_{(\Gamma, g)}\left(\Psi_{\alpha} u_{\alpha}\right) \leq \frac{\lim }{\alpha} \mathcal{E}_{\left(\Gamma_{\alpha}, g_{\alpha}\right)}\left(u_{\alpha}\right)
$$

for any $u_{\alpha} \in C\left(V_{\Gamma_{\alpha}}\right)$ with $\left\|u_{\alpha}\right\|_{L^{2}}=1$ and $\sup _{\alpha} \mathcal{E}_{\left(\Gamma_{\alpha}, g_{\alpha}\right)}\left(u_{\alpha}\right)<\infty$ (see $[22,30])$. Note that $W^{1,2}\left(V_{\Gamma}\right)=L^{2}\left(V_{\Gamma}\right)=C\left(V_{\Gamma}\right)$ holds because of the finiteness of $\Gamma$. Therefore, for any such $\left\{u_{\alpha}\right\},\left\{\Psi_{\alpha} u_{\alpha}\right\}$ is uniformly bounded and then has a convergent subnet $\left\{\Psi_{\beta} u_{\beta}\right\}$. Since $u_{\beta} L^{2}$ strongly converges to $\lim _{\beta} \Psi_{\beta} u_{\beta},\left\{\mathcal{E}_{\left(\Gamma_{\alpha}, g_{\alpha}\right)}\right\}$ is asymptotically compact. Remarking that $\mathcal{E}_{(\Gamma, g)}$ is continuous on $C\left(V_{\Gamma}\right)$ with respect to the $L^{\infty}$ norm, we easily see $(\mathrm{F} 1)$.

We next consider infinite graphs.

Theorem 4.1. Let $\left\{\left(\Gamma_{\alpha}, g_{\alpha}\right)\right\}_{\alpha \in \mathcal{A}} \subset \mathcal{G}$ be a net and $(\Gamma, g) \in \mathcal{G}$ be such that $W^{1,2}\left(V_{\Gamma}\right)=W_{0}^{1,2}\left(V_{\Gamma}\right)$. If $\left(M_{\Gamma_{\alpha}}, \mu_{g_{\alpha}}\right)$ converges to $\left(M_{\Gamma}, \mu_{g}\right)$ with respect to the compact measured GH topology, then the spectral structure $\Sigma\left(\Gamma_{\alpha}, g_{\alpha}\right)$ strongly converges to $\Sigma(\Gamma, g)$ and in particular

$$
\sigma\left(\Delta_{(\Gamma, g)}\right) \subset \lim _{\alpha} \sigma\left(\Delta_{\left(\Gamma_{\alpha}, g_{\alpha}\right)}\right) .
$$

Proof. The theorem is proved in the almost same way as in the proof of Theorem 3.1. Here, the only difference is the definition of $\bar{u}$. There exist measured $\epsilon_{\alpha}$-approximations $f_{\alpha}: M_{\Gamma_{\alpha}} \supset O_{\alpha} \rightarrow f_{\alpha}\left(O_{\alpha}\right) \subset M_{\Gamma}, \epsilon_{\alpha} \searrow 0$, such that

(1) $O_{\alpha}$ and $f_{\alpha}\left(O_{\alpha}\right)$ for each $\alpha$ are geometric images of finite subgraphs of $\Gamma_{\alpha}$ and $\Gamma$ respectively, 
(2) $\left\{f_{\alpha}\left(O_{\alpha}\right)\right\}$ is a monotone increasing net covering $M_{\Gamma}$,

(3) each $f_{\alpha}$ maps vertices to vertices.

Let $\Psi_{\alpha}: C\left(O_{\alpha}\right) \rightarrow C_{0}\left(V_{\Gamma}\right)$ be as in the proof of Proposition 4.1. We now define $\bar{u}_{\alpha}:=\Psi_{\alpha}\left(\left.u_{\alpha}\right|_{O_{\alpha}}\right)$. The rest of the proof is completely same as of Theorem 3.1.

Remark 4.1. The same proofs yield that Proposition 4.1 and Theorem 4.1 are both true for degeneration of graphs in the sense of [30].

\subsection{Negligibility of boundary.}

In this section, we discuss the condition $W^{1,2}\left(V_{\Gamma}\right)=W_{0}^{1,2}\left(V_{\Gamma}\right)$. Let $(\Gamma, g) \in \mathcal{G}$ be an infinite graph with a simplicial metric $g=(\ell, w)$.

Proposition 4.2. If $C_{\Gamma}:=\sup _{x \in V_{\Gamma}} \frac{m\left(E_{x}\right)}{w(x)}<\infty$, we have:

(1) $W^{1,2}\left(V_{\Gamma}\right)=L^{2}\left(V_{\Gamma}\right)$, and moreover $\left.\tilde{\Delta}\right|_{L^{2}\left(V_{\Gamma}\right)}$ and $\left.d\right|_{L^{2}\left(V_{\Gamma}\right)}$ are both bounded linear operators on $L^{2}\left(V_{\Gamma}\right)$,

(2) $W^{1,2}\left(V_{\Gamma}\right)=W_{0}^{1,2}\left(V_{\Gamma}\right)$ and $\Delta=\left.\tilde{\Delta}\right|_{L^{2}\left(V_{\Gamma}\right)}$.

Note that any finite graph satisfies $C_{\Gamma}<\infty$.

Proof. (1): Define $\varphi_{x} \in L^{2}\left(V_{\Gamma}\right), x \in V_{\Gamma}$, by $\varphi_{x}(x):=w(x)^{1 / 2}$ and $\varphi_{x}(y):=0$ for any $y \neq x$. It is easy to see that $\left\{\varphi_{x}\right\}_{x \in V_{\Gamma}}$ is a complete orthonormal basis on $L^{2}\left(V_{\Gamma}\right)$. A straight forward calculation shows that for any $x, y \in V_{\Gamma}$,

$$
\left|\mathcal{E}_{(\Gamma, g)}\left(\varphi_{x}, \varphi_{y}\right)\right|=\frac{m\left(E_{x} \cap E_{y}\right)}{\sqrt{w(x) w(y)}} \leq C_{\Gamma}
$$

and hence, for any $u=\sum_{x \in V_{\Gamma}} a_{x} \varphi_{x}, v=\sum_{x \in V_{\Gamma}} b_{x} \varphi_{x} \in L^{2}\left(V_{\Gamma}\right)$,

$$
\left|(\tilde{\Delta} u, v)_{L^{2}}\right|=\left|\mathcal{E}_{(\Gamma, g)}(u, v)\right| \leq C_{\Gamma} \sum_{x, y \in V_{\Gamma}}\left|a_{x}\right|\left|b_{x}\right| \leq C_{\Gamma}\|u\|_{L^{2}}\|v\|_{L^{2}}
$$

This proves (1).

(2): Assume that $W_{0}^{1,2}\left(V_{\Gamma}\right) \ni u_{i} \rightarrow u \in L^{2}\left(V_{\Gamma}\right)$ as $i \rightarrow \infty$ with respect to the $L^{2}$ norm. Then, (4.1) shows $\mathcal{E}_{(\Gamma, g)}\left(u_{i}-u\right) \rightarrow 0$ and hence $\left\|u_{i}-u\right\|_{W^{1,2}} \rightarrow$ 0 . This completes the proof of the proposition. 
Proposition 4.3. If $W^{1,2}\left(V_{\Gamma}\right)=W_{0}^{1,2}\left(V_{\Gamma}\right)$, then $\left.\tilde{\Delta}\right|_{C_{0}\left(V_{\Gamma}\right)}$ is essentially selfadjoint and its closure coincides with $\Delta_{(\Gamma, g)}$.

Proof. The proof is nothing but a simple modification of that in [25].

Set $d_{0}:=\left.d\right|_{C_{0}\left(V_{\Gamma}\right)}$ and $\delta_{0}:=\left.\delta\right|_{C_{0}\left(E_{\Gamma}\right)}$. Since the adjoint $\delta_{0}^{*}$ is closed and $d_{0} \subset \delta_{0}^{*}$, we have $\bar{d}_{0} \subset \delta_{0}^{*}$.

We shall prove $\delta_{0}^{*} \subset \bar{d}_{0}$. Let $u \in \mathcal{D}\left(\delta_{0}^{*}\right)$. For any $\omega \in \mathcal{D}\left(\delta_{0}\right)=C_{0}\left(E_{\Gamma}\right)$ we have

$$
\int_{e \in E_{\Gamma}}\langle d u(e), \omega(e)\rangle_{g} m_{g}(d e)=\left(u, \delta_{0} \omega\right)_{L^{2}}=\left(\delta_{0}^{*} u, \omega\right)_{L^{2}}
$$

which implies that $d u=\delta_{0}^{*} u \in L^{2}\left(E_{\Gamma}\right)$. Hence, $u \in W^{1,2}\left(V_{\Gamma}\right)=W_{0}^{1,2}\left(V_{\Gamma}\right)=$ $\mathcal{D}\left(\bar{d}_{0}\right)$ and $\bar{d}_{0} u=d u=\delta_{0}^{*} u$. Thus we obtain $\bar{d}_{0}=\delta_{0}^{*}$, which implies the proposition.

\section{Convergence of measured metric spaces with Dirichlet forms.}

\subsection{Preliminaries for Dirichlet form.}

In this section, let us briefly recall the basics for Dirichlet form. Refer to [24] for the details. Let $X$ be a locally compact separable metric space and $m$ a positive Radon measure with full support. We consider a symmetric nonnegative definite bilinear form $\mathcal{E}$ defined on a (not necessarily dense) linear domain $\mathcal{D}(\mathcal{E}) \subset L^{2}(X ; m)$. We define an inner product $\mathcal{E}_{1}$ on $\mathcal{D}(\mathcal{E})$ by

$$
\mathcal{E}_{1}(u, v):=\mathcal{E}(u, v)+(u, v)_{L^{2}(X ; m)}, \quad u, v \in \mathcal{D}(\mathcal{E}) .
$$

The pair $(\mathcal{E}, \mathcal{D}(\mathcal{E}))$ or simply $\mathcal{E}$ is said to be a Dirichlet form on $L^{2}(X ; m)$ if it is closed (i.e., $\mathcal{D}(\mathcal{E})$ is complete with respect to the $\mathcal{E}_{1}^{1 / 2}$-norm) and if it is Markovian (i.e., for any $u \in \mathcal{D}(\mathcal{E})$ we have $u^{\sharp}:=0 \vee u \wedge 1 \in \mathcal{D}(\mathcal{E})$ and $\left.\mathcal{E}\left(u^{\sharp}, u^{\sharp}\right) \leq \mathcal{E}(u, u)\right)$. A Dirichlet form $\mathcal{E}$ densely defined on $L^{2}(X ; m)$ is said to be regular if $\mathcal{D}(\mathcal{E}) \cap C_{0}(X)$ is dense in $\mathcal{D}(\mathcal{E})$ with respect to the $\mathcal{E}_{1}^{1 / 2}$-norm and dense in $C_{0}(X)$ with respect to the uniform norm. A Dirichlet form $\mathcal{E}$ on $L^{2}(X ; m)$ is said to be local if $\mathcal{E}(u, v)=0$ for any $u, v \in \mathcal{D}(\mathcal{E})$ such that $\operatorname{supp} u$ and $\operatorname{supp} v$ are disjoint compact subsets. A Dirichlet form $\mathcal{E}$ on $L^{2}(X ; m)$ is said to be strongly local if $\mathcal{E}(u, v)=0$ for any $u, v \in \mathcal{D}(\mathcal{E})$ such that $\operatorname{supp} u$ and $\operatorname{supp} v$ are compact and $u=$ const $m$-a.e. on a neighborhood of $\operatorname{supp} v$. It follows from Theorem 4.5.3 and Lemma 5.3.3 of [24] that a regular Dirichlet form $\mathcal{E}$ on $L^{2}(X ; m)$ is local (resp. strongly local) if and 
only if $\mathcal{E}(u, v)=0$ for any $u, v \in \mathcal{D}(\mathcal{E})$ such that $u=0$ (resp. $u=$ const) $m$-a.e. on a neighborhood of $\operatorname{supp} v$.

Let $\mathcal{E}$ be a strongly local regular Dirichlet form on $L^{2}(X ; m)$. The 1 capacity $\operatorname{Cap}(\cdot)$ over $X$ with respect to $\mathcal{E}$ is defined as follows: for any open subset $O$ of $X$,

$$
\operatorname{Cap}(O):=\inf \left\{\mathcal{E}_{1}(u, u) \mid u \in \mathcal{D}(\mathcal{E}), u \geq 1 m \text {-a.e. on } O\right\}
$$

and for any subset $A$ of $X$,

$$
\operatorname{Cap}(A):=\inf \{\operatorname{Cap}(O) \mid O \text { is open set with } A \subset O\} .
$$

A quasi-continuous m-version $\tilde{u}$ of $u \in \mathcal{D}(\mathcal{E})$ is a function satisfying that $\tilde{u}=u m$-a.e. and that there exists a sequence $\left\{F_{k}\right\}$ of closed subsets of $X$ such that $\lim _{k \rightarrow \infty} \operatorname{Cap}\left(X \backslash F_{k}\right)=0$ and each $\left.\tilde{u}\right|_{F_{k}}$ is continuous on $F_{k}$. It is known that any $u \in \mathcal{D}(\mathcal{E})$ admits a quasi-continuous $m$-version (see [24]).

For any $u, v \in \mathcal{D}(\mathcal{E})$, there exists a unique signed finite Borel measure $\Gamma(u, v)$ on $X$, called the energy measure of $\mathcal{E}$, charging no set of zero 1capacity, such that

$$
\begin{aligned}
& 2 \int_{X} \tilde{w} d \Gamma(u, v)=\mathcal{E}(u w, v)+\mathcal{E}(u, v w)-\mathcal{E}(u v, w), \\
& u, v, w \in \mathcal{D}(\mathcal{E}) \cap L^{\infty}(X ; m), \\
& \mathcal{E}(u, v)=\Gamma(u, v)(X), \quad u, v \in \mathcal{D}(\mathcal{E}) .
\end{aligned}
$$

We set $\Gamma(u):=\Gamma(u, u)$ for $u \in \mathcal{D}(\mathcal{E})$. Note that the energy measure of the canonical Dirichlet form $\mathcal{E}_{M}$ of a Riemannian manifold $M$ coincides with $d \Gamma(u, v)=\langle d u, d v\rangle_{M} d \operatorname{vol}_{M}$ for $u, v \in W_{0}^{1,2}(M)$.

Lemma 5.1. For any $u, v \in \mathcal{D}(\mathcal{E}) \cap L^{\infty}(X ; m)$ we have

$$
\mathcal{E}(u v)^{1 / 2} \leq\left(\int_{X} \tilde{u}^{2} d \Gamma(u)\right)^{1 / 2}+\left(\int_{X} \tilde{v}^{2} d \Gamma(v)\right)^{1 / 2} .
$$

Proof. Lemma 3.2.5 of [24] implies that

$$
d \Gamma(u v)=\tilde{v}^{2} d \Gamma(u)+2 \tilde{u} \tilde{v} d \Gamma(u, v)+\tilde{u}^{2} d \Gamma(v) .
$$

Applying Lemma 5.6.1 of [24] yields

$$
\left(\int_{X} \tilde{u} \tilde{v} d \Gamma(u, v)\right)^{2} \leq \int_{X} \tilde{u}^{2} d \Gamma(u) \int_{X} \tilde{v}^{2} d \Gamma(v) .
$$


Combining these two formulas completes the proof.

The intrinsic (or Carathéodory) metric $d_{\mathcal{E}}: X \times X \rightarrow[0, \infty]$ induced from $\mathcal{E}$ is defined by

$$
d_{\mathcal{E}}(x, y):=\sup \left\{u(x)-u(y) \mid u \in \mathcal{D}(\mathcal{E})_{\operatorname{loc}} \cap C(X), \Gamma(u) \leq m\right\}
$$

for $x, y \in X$, where $\mathcal{D}(\mathcal{E})_{\text {loc }}$ denotes the localization of $\mathcal{D}(\mathcal{E})$. It follows that $d_{\mathcal{E}}$ is a pseudo-distance function on $X$. According to $[52,53]$, if $d_{\mathcal{E}}$ coincides with the original metric on $X$, then

- it is a length metric,

- $X$ is complete if and only if any bounded subset is relatively compact.

\subsection{Asymptotic compactness of Dirichlet forms.}

We denote by const $a, b, \ldots$ some constant depending only on $a, b, \ldots$ Let $\mathcal{M D}_{c}$ be the set of all $(X, p, m, \mathcal{E})$, where $(X, p, m) \in \mathcal{M}_{c}$ (defined in 2.1) and $\mathcal{E}$ is any strongly local regular Dirichlet form on $L^{2}(X ; m)$. For a net $\left\{\left(X_{\alpha}, p_{\alpha}, m_{\alpha}, \mathcal{E}_{\alpha}\right)\right\}_{\alpha \in \mathcal{A}} \subset \mathcal{M D}_{c}$, we consider the following conditions.

(N) For any $R>r>0$, any sufficiently large $\alpha$ compared with $R, r$, and for any $x \in X_{\alpha}$, the number of elements of any $r$-discrete net in $\bar{B}(x, R)$ is at most const $r / R$.

(D) We have

$$
\varlimsup_{\alpha} \operatorname{diam} X_{\alpha}<\infty
$$

(M) For each $r, R>0$,

$$
\varlimsup_{\alpha} m_{\alpha}\left(B\left(p_{\alpha}, r\right)\right)<\infty \quad \text { and } \quad \underline{\lim }_{\alpha} \inf _{x \in B\left(p_{\alpha}, R\right)} m_{\alpha}(B(x, r))>0 .
$$

(C) The intrinsic metric $d_{\mathcal{E}_{\alpha}}$ of $\mathcal{E}_{\alpha}$ coincides with the original distance function on $X_{\alpha}$ for any $\alpha$.

(P) There exists a sequence $r_{j} \searrow 0, j=1,2, \ldots$, such that for any $R>0$ and $j \geq 1$ there is $\alpha_{R, j} \in \mathcal{A}$ such that for each $\alpha \geq \alpha_{R, j}$ we can find an $r_{j}$-discrete $r_{j}$-net $\left\{x_{j k}^{\alpha}\right\}_{k}$ of $B\left(p_{\alpha}, R\right)$ satisfying

$$
\left\|u-\bar{u}_{j k}^{\alpha}\right\|_{L^{2}\left(B\left(x_{j k}^{\alpha}, r_{j}\right) ; m\right)} \leq \operatorname{const}_{R} \cdot r_{j}\left(\int_{B\left(x_{j k}^{\alpha}, \text { const } r_{j}\right)} d \Gamma_{\alpha}(u)\right)^{1 / 2}
$$


for any $k$ and $u \in \mathcal{D}\left(\mathcal{E}_{\alpha}\right)$, where $\bar{u}_{j k}^{\alpha}$ is some constant depending only on $u, j, k$, and $\alpha$.

The purpose of this section is to prove the two following theorems:

Theorem 5.1. Let a net $\left\{\left(X_{\alpha}, p_{\alpha}, m_{\alpha}, \mathcal{E}_{\alpha}\right)\right\}_{\alpha \in \mathcal{A}} \subset \mathcal{M D}_{c}$ satisfy (N), (D), $(\mathrm{M})$, and $(\mathrm{P})$. Then, there exists a subnet of $\mathcal{A}$ for which $\left(X_{\alpha}, p_{\alpha}, m_{\alpha}\right)$ measured $G H$ converges to some $(X, p, m) \in \mathcal{M}_{c}$ and the Dirichlet form $\mathcal{E}_{\alpha}$ compactly converges to some Dirichlet form on $L^{2}(X ; m)$.

Theorem 5.2. Let a net $\left\{\left(X_{\alpha}, p_{\alpha}, m_{\alpha}, \mathcal{E}_{\alpha}\right)\right\}_{\alpha \in \mathcal{A}} \subset \mathcal{M D}_{c}$ satisfies (N), (M), $(\mathrm{C})$, and $(\mathrm{P})$. Then, there exists a subnet of $\mathcal{A}$ for which $\left(X_{\alpha}, p_{\alpha}, m_{\alpha}\right)$ measured $G H$ converges to some $(X, p, m) \in \mathcal{M}_{c}$ and the Dirichlet form $\mathcal{E}_{\alpha}$ Mosco converges to some Dirichlet form on $L^{2}(X ; m)$.

Note that in Theorems 5.1 and 5.2, the limit Dirichlet form is not necessarily densely defined on $L^{2}(X ; m)$. For instance, if $\mathcal{E}$ is a densely defined Dirichlet form on $L^{2}(X ; m)$, then, as $r \rightarrow \infty$, the rescaled Dirichlet form $r \mathcal{E}$ converges to the Dirichlet form $\hat{\mathcal{E}}$ defined by

$$
\hat{\mathcal{E}}(u):=0, \quad u \in \mathcal{D}(\hat{\mathcal{E}})=\operatorname{Ker} \mathcal{E}
$$

which is not densely defined in general.

The following is an immediate consequence of Theorem 5.1.

Corollary 5.1. Let $X$ be a compact metric space, $m$ a positive finite Borel measure on $X$ with full support, and $\mathcal{E}$ a strongly local regular Dirichlet form on $L^{2}(X ; m)$. Assume that there exists a sequence $r_{j} \searrow 0, j=1,2, \ldots$, such that for any $j$ there is an $r_{j}$-discrete $r_{j}$-net $\left\{x_{j k}\right\}_{k}$ of $X$ satisfying

$$
\left\|u-\bar{u}_{j k}\right\|_{L^{2}\left(B\left(x_{j k}, r_{j}\right) ; m\right)} \leq \mathrm{const} \cdot r_{j}\left(\int_{B\left(x_{j k}, \mathrm{const} r_{j}\right)} d \Gamma(u)\right)^{1 / 2}
$$

for any $k$ and $u \in \mathcal{D}(\mathcal{E})$, where $\bar{u}_{j k}$ is some constant depending only on $u, j$, and $k$. Then, the embedding $\left(\mathcal{D}(\mathcal{E}), \mathcal{E}_{1}\right) \hookrightarrow L^{2}(X ; m)$ is compact.

If we assume $(\mathrm{N}),(\mathrm{D}),(\mathrm{M}),(\mathrm{C})$, the doubling condition for measure:

$$
\frac{m_{\alpha}(B(x, 2 r))}{m_{\alpha}(B(x, r))} \leq \mathrm{const}
$$


and the weak Poincaré inequality on balls:

$$
\left\|u-\bar{u}_{x, r}\right\|_{L^{2}(B(x, r) ; m)} \leq \mathrm{const} \cdot r\left(\int_{B(x, \mathrm{const} r)} d \Gamma_{\alpha}(u)\right)^{1 / 2}
$$

for any $x \in X_{\alpha}, r \in(0$, const $)$, and $\alpha \in \mathcal{A}$, where $\bar{u}_{x, r}$ is the average of $u$ on $B(x, r)$, then the conclusion of Theorem 5.1 can also be obtained by the method of Kasue and Kumura [33] using heat kernels. Under the assumption of Theorem 5.1 or 5.2, the heat kernel bound in [33] cannot be obtained in general - even the heat kernel (i.e., the integral kernel of the semigroup) does not necessarily exist. Under the doubling condition for measure and the weak Poincaré inequality on balls for $(X, m, \mathcal{E})$ as in Corollary 5.1 , the compactness of the embedding $\left(\mathcal{D}(\mathcal{E}), \mathcal{E}_{1}\right) \hookrightarrow L^{2}(X ; m)$ is well-known. The corollary says that this holds without the doubling condition for measure.

We apply Theorem 5.1 to prove Theorem 3.2. Theorem 5.2 has an application to convergence of complete noncompact Riemannian manifolds with a uniform lower bound of Ricci curvature (see $\S 5.3$ ).

Let us first discuss the conditions (N), (D), and (M). If $\left\{\left(X_{\alpha}, p_{\alpha}\right)\right\}_{\alpha \in \mathcal{A}}$ is GH convergent, we have $(\mathrm{N})$. In this case, (D) is equivalent to the compactness of the limit. If $\left\{\left(X_{\alpha}, p_{\alpha}, m_{\alpha}\right)\right\}_{\alpha \in \mathcal{A}}$ is measured GH convergent, then the first formula of (M) holds and the second is equivalent to the fullness of the support of the limit measure. Conversely, it is well-known (see [28]) that $(\mathrm{N})$ implies the existence of a subnet of $\left\{\left(X_{\alpha}, p_{\alpha}\right)\right\}$ which converges in $\mathcal{M e t}_{c}$ with respect to the GH topology. This together with Corollary 2.1 leads to the following:

Lemma 5.2. If a net $\left\{\left(X_{\alpha}, p_{\alpha}, m_{\alpha}\right)\right\}_{\alpha \in \mathcal{A}} \subset \mathcal{M}_{c}$ satisfies $(\mathrm{N})$ and $(\mathrm{M})$, then there exists a measured $G H$ convergent subnet of $\left\{\left(X_{\alpha}, p_{\alpha}, m_{\alpha}\right)\right\}$ in $\mathcal{M}_{c}$ whose limit measure has full support.

Let us give a net $\left\{\left(X_{\alpha}, p_{\alpha}, m_{\alpha}, \mathcal{E}_{\alpha}\right)\right\}_{\alpha \in \mathcal{A}} \subset \mathcal{M D}_{c}$. By Lemma 5.2, we may suppose the following for the proof of Theorems 5.1 and 5.2.

Assumption 5.1. The net $\left\{\left(X_{\alpha}, p_{\alpha}, m_{\alpha}\right)\right\}$ measured GH converges to a space $(X, p, m) \in \mathcal{M}_{c}$ such that $m$ has full support.

Under this assumption, we shall show some lemmas.

Lemma 5.3. If a net $u_{\alpha} \in L^{2}\left(X_{\alpha} ; m_{\alpha}\right) L^{2}$ strongly converges to a function $u \in L^{2}(X ; m)$, then $u_{\alpha}^{\sharp} \rightarrow u^{\sharp} L^{2}$ strongly. 
Proof. Assume that $L^{2}\left(X_{\alpha} ; m_{\alpha}\right) \ni u_{\alpha} \rightarrow u \in L^{2}(X ; m) L^{2}$ strongly. Then, there is a net $\tilde{u}_{\beta} \in C_{0}(X)$ such that $\tilde{u}_{\beta} \rightarrow u$ in $L^{2}(X ; m)$ and

$$
\lim _{\beta} \varlimsup_{\alpha}\left\|\Phi_{\alpha} \tilde{u}_{\beta}-u_{\alpha}\right\|_{L^{2}}=0,
$$

where $\Phi_{\alpha}: L^{2}(X ; m) \rightarrow L^{2}\left(X_{\alpha} ; m_{\alpha}\right)$ is defined in Definition 2.3. Remarking that $\left|x^{\sharp}-y^{\sharp}\right| \leq|x-y|$ for any $x, y \in \mathbb{R}$, we have $\left\|\tilde{u}_{\beta}^{\sharp}-u^{\sharp}\right\|_{L^{2}} \leq\left\|\tilde{u}_{\beta}-u\right\|_{L^{2}} \rightarrow 0$ and

$$
\left\|\Phi_{\alpha} \tilde{u}_{\beta}^{\sharp}-u_{\alpha}^{\sharp}\right\|_{L^{2}}=\left\|\left(\Phi_{\alpha} \tilde{u}_{\beta}\right)^{\sharp}-u_{\alpha}^{\sharp}\right\|_{L^{2}} \leq\left\|\Phi_{\alpha} \tilde{u}_{\beta}-u_{\alpha}\right\|_{L^{2}},
$$

which completes the proof.

Lemma 5.4. If $\mathcal{E}_{\alpha}$ is $\Gamma$-convergent, then the $\Gamma$-limit is a Dirichlet form on $L^{2}(X ; m)$.

Proof. Assume that $\mathcal{E}_{\alpha} \Gamma$-converges to $\mathcal{E}$. Then, by Lemma $2.11, \mathcal{E}$ is a closed bilinear form on $L^{2}(X ; m)$. It suffices to show the Markovian property of $\mathcal{E}$. Take any $u \in \mathcal{D}(\mathcal{E})$ and fix it. By $(\mathrm{F} 2)$, there exists a net $u_{\alpha} \in \mathcal{D}\left(\mathcal{E}_{\alpha}\right)$ such that $u_{\alpha} \rightarrow u L^{2}$ strongly and $\mathcal{E}_{\alpha}\left(u_{\alpha}\right) \rightarrow \mathcal{E}(u)$. Lemma 5.3 implies that $u_{\alpha}^{\sharp} \rightarrow u^{\sharp} L^{2}$ strongly. Hence, from (F1) and the Markovian property of $\mathcal{E}_{\alpha}$,

$$
\mathcal{E}\left(u^{\sharp}\right) \leq \frac{\lim }{\alpha} \mathcal{E}_{\alpha}\left(u_{\alpha}^{\sharp}\right) \leq \lim _{\alpha} \mathcal{E}_{\alpha}\left(u_{\alpha}\right)=\mathcal{E}(u),
$$

which completes the proof of Lemma 5.4.

In addition to Assumption 5.1, we assume (P). For any $\alpha \in \mathcal{A}$ and $R>0$, we denote by $\mathcal{E}_{\alpha}^{R}$ the restriction of $\mathcal{E}_{\alpha}$ on the $\mathcal{E}_{\alpha, 1^{-}}$closure, say $\mathcal{D}\left(\mathcal{E}_{\alpha}^{R}\right)$, of the set of $u \in \mathcal{D}\left(\mathcal{E}_{\alpha}\right)$ with $\operatorname{supp} u \subset B\left(p_{\alpha}, R\right)$. Note that we have $\left(\mathcal{E}_{\alpha}^{R}, \mathcal{D}\left(\mathcal{E}_{\alpha}^{R}\right)\right)=$ $\left(\mathcal{E}_{\alpha}, \mathcal{D}\left(\mathcal{E}_{\alpha}\right)\right)$ for sufficiently large $R>0$ provided (D) is satisfied. Let us fix $R>0, j \geq 1$, and $\alpha \geq \alpha_{R, j}$, where $\alpha_{R, j}$ is as in (P). By taking a $r_{j}$-discrete $r_{j}$-net $\left\{x_{j k}^{\alpha}\right\}_{k=1}^{N_{j}^{\alpha}}$ of $\bar{B}\left(p_{\alpha}, R\right)$ as in $(\mathrm{P})$, it follows from (N) that $N_{j}^{\alpha} \leq$ const $_{r_{j} / R}$. For any $u \in \mathcal{D}\left(\mathcal{E}_{\alpha}^{R}\right)$, we define a function $\bar{u}_{j}^{\alpha}: X_{\alpha} \rightarrow \mathbb{R}$ by $\bar{u}_{j}^{\alpha}:=\bar{u}_{j k}^{\alpha}$ on each $U_{j k}^{\alpha}:=B\left(x_{j k}^{\alpha}, r_{j}\right) \backslash \bigcup_{\ell=1}^{k-1} B\left(x_{j \ell}^{\alpha}, r_{j}\right)$ and $\bar{u}_{j}^{\alpha}:=0$ on $X \backslash \bigcup_{k=1}^{N_{j}^{\alpha}} U_{j k}^{\alpha}$. Note that $U_{j k}^{\alpha} \cap U_{j \ell}^{\alpha}=\emptyset$ for any $k \neq \ell$ and that $\bar{B}\left(p_{\alpha}, R\right) \subset$ $\bigcup_{k=1}^{N_{j}^{\alpha}} U_{j k}^{\alpha} \subset B\left(p_{\alpha}, R+r_{j}\right)$.

Lemma 5.5. We have

$$
\left\|u-\bar{u}_{j}^{\alpha}\right\|_{L^{2}\left(X_{\alpha} ; m_{\alpha}\right)} \leq \operatorname{const}_{R} \cdot r_{j}\left(\int_{B\left(p_{\alpha}, R+\operatorname{const} r_{j}\right)} d \Gamma_{\alpha}(u)\right)^{1 / 2} .
$$


Proof. By (P),

$$
\begin{aligned}
\left\|u-\bar{u}_{j}^{\alpha}\right\|_{L^{2}\left(X_{\alpha} ; m_{\alpha}\right)}^{2} & =\sum_{k=1}^{N_{j}^{\alpha}}\left\|u-\bar{u}_{j k}^{\alpha}\right\|_{L^{2}\left(U_{j k}^{\alpha} ; m_{\alpha}\right)}^{2} \\
& \leq \operatorname{const}_{R} \cdot r_{j}^{2} \sum_{k=1}^{N_{j}^{\alpha}} \int_{B\left(x_{j k}^{\alpha}, \operatorname{const} r_{j}\right)} d \Gamma_{\alpha}(u) .
\end{aligned}
$$

It follows from $(\mathrm{N})$ that $\#\left\{k \mid d\left(x, x_{j k}^{\alpha}\right)<\right.$ const $\left.r_{j}\right\}$ is bounded uniformly for $x \in X$ and large $\alpha$. Therefore, the right hand side of the above is

$$
\leq \operatorname{const}_{R} \cdot r_{j}^{2} \int_{B\left(p_{\alpha}, R+\text { const } r_{j}\right)} d \Gamma_{\alpha}(u)
$$

This completes the proof.

For convenience in the later discussions, we define two functions $L_{+}(x, a, b): S \rightarrow[0,1]$ and $L_{-}(x, a, b): S \rightarrow[0,1]$ for $a<b$ and $x \in S \in \mathcal{M e t}_{c}$ by

$$
L_{+}(x, a, b)(y):=\left(\frac{d(x, y)-a}{b-a}\right)^{\sharp}, \quad L_{-}(x, a, b)(y):=\left(\frac{b-d(x, y)}{b-a}\right)^{\sharp}
$$

for any $y \in S$, where $d$ is the distance function on $S$. Then, $L_{ \pm}(x, a, b)$ are Lipschitz functions on $S$ with Lipschitz constant $1 /(b-a)$.

Theorem 5.1 is derived from Lemma 5.4, Corollary 2.3, and the following:

Theorem 5.3. Under Assumption 5.1 and $(\mathrm{P})$, the net $\left\{\mathcal{E}_{\alpha}^{R}\right\}_{\alpha \in \mathcal{A}}$ is asymptotically compact for each fixed $R>0$.

Proof. Fix an $R>0$ and let $u_{\alpha} \in \mathcal{D}\left(\mathcal{E}_{\alpha}^{R}\right)$ be such that $\sup _{\alpha} \mathcal{E}_{\alpha, 1}\left(u_{\alpha}\right)<\infty$. It suffices to prove that $\left\{u_{\alpha}\right\}_{\alpha \in \mathcal{A}}$ has an $L^{2}$ convergent subnet. There are measured $\epsilon_{\alpha}$-approximations $f_{\alpha}:\left(B\left(p_{\alpha}, t_{\alpha}\right), p_{\alpha}\right) \rightarrow\left(B\left(p, t_{\alpha}^{\prime}\right), p\right)$ with $\epsilon_{\alpha} \searrow 0$ and $t_{\alpha}, t_{\alpha}^{\prime} \nearrow \infty$. For any $j \geq 1$ and $\alpha \geq \alpha_{R, j}$, let $x_{j k}^{\alpha}$ and $U_{j k}^{\alpha}, k=1, \ldots, N_{j}^{\alpha}$, be as above. There is a subnet $\mathcal{A}_{j}$ of $\mathcal{A}$ depending on $j$ such that for every $k=1, \ldots, N_{j}^{\alpha}$, the limits $x_{j k}:=\lim _{\alpha} f_{\alpha}\left(x_{j k}^{\alpha}\right), N_{j}:=\lim _{\alpha} N_{j}^{\alpha}$, and $c_{j k}:=\lim _{\alpha} \bar{u}_{j k}^{\alpha}$ all exist. Replacing with a subnet of $\mathcal{A}_{j}$, we assume that $N_{j}=N_{j}^{\alpha}$ for all $\alpha \in \mathcal{A}_{j}$. We may also assume that $\mathcal{A}_{j+1} \subset \mathcal{A}_{j}$ for any $j \geq 1$. Therefore, by a diagonal argument, we find a common cofinal subnet of all 
$\mathcal{A}_{j}$ and write it also by $\mathcal{A}$. Set $U_{j k}:=B\left(x_{j k}, r_{j}\right) \backslash \bigcup_{\ell=1}^{k-1} B\left(x_{j \ell}, r_{j}\right)$. For any $\epsilon>0, x \in \bar{B}(p, R)$, and any set $A$, we define

$$
\begin{aligned}
\chi_{B\left(x, r_{j}\right)}^{\epsilon} & :=L_{-}\left(x, r_{j}-2 \epsilon, r_{j}-\epsilon\right): X \rightarrow[0,1], \\
\chi_{U_{j k}}^{\epsilon} & :=\chi_{B\left(x_{j k}, r_{j}\right)}^{\epsilon} \cdot \prod_{\ell=1}^{k-1}\left(1-\chi_{B\left(x_{j \ell}, r_{j}\right)}^{\epsilon}\right): X \rightarrow[0,1], \\
I_{A}(x) & := \begin{cases}1 & \text { if } x \in A, \\
0 & \text { if } x \notin A .\end{cases}
\end{aligned}
$$

Claim 5.1. We have

$$
\begin{gathered}
\lim _{\epsilon \searrow 0}\left\|\chi_{U_{j k}}^{\epsilon}-I_{U_{j k}}\right\|_{L^{2}(X ; m)}=0, \\
\lim _{\epsilon \searrow 0} \lim _{\alpha}\left\|\Phi_{\alpha} \chi_{U_{j k}}^{\epsilon}-I_{U_{k}^{\alpha}}\right\|_{L^{2}\left(X_{\alpha} ; m_{\alpha}\right)}=0
\end{gathered}
$$

for any $j=1,2, \ldots$ and $k=1, \ldots, N_{j}$, where $\Phi_{\alpha}$ is associated with $f_{\alpha}$.

Proof. We set $A(x, r, R):=B(x, R) \backslash B(x, r)$. Since $\left\{I_{U_{j k}} \neq \chi_{U_{j k}}^{\epsilon}\right\} \subset$ $\bigcup_{\ell=1}^{N_{j}} A\left(x_{j \ell}, r_{j}-2 \epsilon, r_{j}-\epsilon\right)$, we have

$$
\left\|I_{U_{j k}}-\chi_{U_{j k}}^{\epsilon}\right\|_{L^{2}(X ; m)} \leq \sum_{\ell=1}^{N_{j}} m\left(A\left(x_{j \ell}, r_{j}-2 \epsilon, r_{j}-\epsilon\right)\right),
$$

which implies (5.1).

Let $\alpha \in \mathcal{A}$ be sufficient large compared with an arbitrarily fixed $\epsilon>0$. For any $y \in B\left(p_{\alpha}, t_{\alpha}\right)$ and $\ell=1, \ldots, N_{j}$, we have

$$
\left|d\left(x_{j \ell}^{\alpha}, f_{\alpha}(y)\right)-d\left(x_{j \ell}, y\right)\right|<\epsilon_{\alpha}<\epsilon / 2
$$

and hence $\left\{I_{U_{j k}^{\alpha}} \neq \chi_{U_{j k}}^{\epsilon} \circ f_{\alpha}\right\} \subset \bigcup_{\ell=1}^{N_{j}} A_{\ell \epsilon}^{\alpha}$, where $A_{\ell \epsilon}^{\alpha}:=A\left(x_{j \ell}^{\alpha}, r_{j}-3 \epsilon, r_{j}-\right.$ $\epsilon / 2)$, so that

$$
\left\|I_{U_{j k}^{\alpha}}-\chi_{U_{j k}}^{\epsilon} \circ f_{\alpha}\right\|_{L^{2}\left(B\left(p_{\alpha}, t_{\alpha}\right) ; m_{\alpha}\right)} \leq \sum_{\ell=1}^{N_{j}} m\left(A_{\ell \epsilon}^{\alpha}\right) .
$$

Setting

$$
\varphi_{\ell \epsilon}:=L_{-}\left(x_{j \ell}, r_{j}-\epsilon / 4, r_{j}-\epsilon / 8\right) \cdot L_{+}\left(x_{j \ell}, r_{j}-5 \epsilon, r_{j}-4 \epsilon\right): X \rightarrow[0,1],
$$


we have $I_{A_{\ell \epsilon}^{\alpha}} \leq \varphi_{\ell \epsilon} \circ f_{\alpha}$ for large $\alpha$ and so

$$
\varlimsup_{\alpha} m\left(A_{\ell \epsilon}^{\alpha}\right) \leq \varlimsup_{\alpha} \int_{B\left(p_{\alpha}, t_{\alpha}\right)} \varphi_{\ell \epsilon} \circ f_{\alpha} d m_{\alpha}=\int_{X} \varphi_{\ell \epsilon} d m,
$$

which tends to zero as $\epsilon \rightarrow 0$. This completes the proof of the claim.

Let $\bar{u}_{j}^{\alpha}$ be as above, and define two functions on $X$ by

$$
\bar{u}_{j}:=\sum_{k=1}^{N_{j}} c_{j k} I_{U_{j k}} \quad \text { and } \quad \tilde{u}_{j}^{\epsilon}:=\sum_{k=1}^{N_{j}} c_{j k} \chi_{U_{j k}}^{\epsilon} .
$$

Claim 5.2. For any $j=1,2, \ldots$ we have

$$
\begin{gathered}
\lim _{\epsilon \searrow 0}\left\|\tilde{u}_{j}^{\epsilon}-\bar{u}_{j}\right\|_{L^{2}(X ; m)}=0, \\
\lim _{\epsilon \searrow 0} \varlimsup_{\alpha}\left\|\Phi_{\alpha} \tilde{u}_{j}^{\epsilon}-\bar{u}_{j}^{\alpha}\right\|_{L^{2}\left(X_{\alpha} ; m_{\alpha}\right)}=0 .
\end{gathered}
$$

Consequently, $\lim _{\alpha} \bar{u}_{j}^{\alpha}=\bar{u}_{j}\left(L^{2}\right.$ strongly).

Proof. The claim directly follows from Claim 5.1.

Claim 5.3. The sequence $\left\{\bar{u}_{j}\right\}$ is Cauchy in $L^{2}(X ; m)$.

Proof. By Claim 5.2, for any $j$ and $j^{\prime}$,

$$
\begin{aligned}
&\left\|\bar{u}_{j}-\bar{u}_{j^{\prime}}\right\|_{L^{2}(X ; m)}= \lim _{\epsilon \searrow 0}\left\|\tilde{u}_{j}^{\epsilon}-\tilde{u}_{j^{\prime}}^{\epsilon}\right\|_{L^{2}(X ; m)} \\
&= \lim _{\epsilon \searrow 0} \lim _{\alpha}\left\|\Phi_{\alpha} \tilde{u}_{j}^{\epsilon}-\Phi_{\alpha} \tilde{u}_{j^{\prime}}^{\epsilon}\right\|_{L^{2}\left(X_{\alpha} ; m_{\alpha}\right)} \\
&=\varlimsup_{\alpha}\left\|\bar{u}_{j}^{\alpha}-\bar{u}_{j^{\prime}}^{\alpha}\right\|_{L^{2}\left(X_{\alpha} ; m_{\alpha}\right)} \\
& \leq \varlimsup_{\alpha}\left(\left\|\bar{u}_{j}^{\alpha}-u_{\alpha}\right\|_{L^{2}\left(X_{\alpha} ; m_{\alpha}\right)}\right. \\
&\left.\quad+\left\|u_{\alpha}-\bar{u}_{j^{\prime}}^{\alpha}\right\|_{L^{2}\left(X_{\alpha} ; m_{\alpha}\right)}\right),
\end{aligned}
$$

which tends to zero as $j, j^{\prime} \rightarrow \infty$ because of Lemma 5.5. This shows the claim.

Let $u:=\lim _{j \rightarrow \infty} \bar{u}_{j} \in L^{2}(X ; m)$. We prove that $u_{\alpha} \rightarrow u L^{2}$ strongly in the following. In fact, there exists a sequence $\epsilon_{j} \searrow 0$ such that $\lim _{j} \tilde{u}_{j}^{\epsilon_{j}}=u$ 
and

$$
\begin{aligned}
& \varlimsup_{j} \varlimsup_{\alpha}\left\|\Phi_{\alpha} \tilde{u}_{j}^{\epsilon_{j}}-u_{\alpha}\right\|_{L^{2}\left(X_{\alpha} ; m_{\alpha}\right)} \\
& \leq \varlimsup_{j} \varlimsup_{\epsilon \searrow 0} \varlimsup_{\alpha}\left\|\Phi_{\alpha} \tilde{u}_{j}^{\epsilon}-u_{\alpha}\right\|_{L^{2}\left(X_{\alpha} ; m_{\alpha}\right)} .
\end{aligned}
$$

By Claim 5.2, the right hand side of the above inequality is

$$
\leq \varlimsup_{j} \varlimsup_{\alpha}\left\|\bar{u}_{j}^{\alpha}-u_{\alpha}\right\|_{L^{2}\left(X_{\alpha} ; m_{\alpha}\right)}=0 .
$$

This completes the proof of Theorem 5.3.

Under Assumption 5.1, we have a $\Gamma$-convergent subnet of $\left\{\mathcal{E}_{\alpha}\right\}$ by Theorem 2.3. Assume that $\mathcal{E}_{\alpha} \Gamma$-converges to a Dirichlet form $\mathcal{E}$ on $L^{2}(X ; m)$. Supposing (P), Theorem 5.3, Corollary 2.3, and Lemma 5.4 together show the existence of a subnet $\left\{\mathcal{E}_{\alpha}^{R}\right\}_{\alpha \in \mathcal{A}_{R}}$ depending on $R>0$ which compactly converges to a Dirichlet form $\mathcal{E}^{R}$ on $L^{2}(X ; m)$. The following is obvious.

Lemma 5.6. We have $\mathcal{D}\left(\mathcal{E}^{R}\right) \subset \mathcal{D}(\mathcal{E})$ and $\mathcal{E}^{R}(u)=\mathcal{E}(u)$ for any $u \in \mathcal{D}\left(\mathcal{E}^{R}\right)$, $R>0$.

With these preparations, we finally give:

Proof of Theorem 5.2. Assume that $L^{2}\left(X_{\alpha} ; m_{\alpha}\right) \ni u_{\alpha} \rightarrow u \in L^{2}(X ; m) L^{2}$ weakly and $\sup _{\alpha} \mathcal{E}_{\alpha, 1}\left(u_{\alpha}\right)<\infty$. It suffices to prove (F1'), i.e.,

$$
\mathcal{E}(u) \leq \varlimsup_{\alpha \in \mathcal{A}} \mathcal{E}_{\alpha}\left(u_{\alpha}\right)
$$

Since each $u_{\alpha}$ can be $\mathcal{E}_{\alpha, 1}$-approximated by a bounded function, we may assume that each $u_{\alpha}$ is bounded.

Let $f_{\alpha}:\left(B\left(p_{\alpha}, t_{\alpha}\right), p_{\alpha}\right) \rightarrow\left(B\left(p, t_{\alpha}^{\prime}\right), p\right)$ be measured $\epsilon_{\alpha}$-approximations with $\epsilon_{\alpha} \searrow 0$ and $t_{\alpha}, t_{\alpha}^{\prime} \nearrow \infty$, and $\Phi_{\alpha}$ be as in Definition 2.3. We set $\varphi_{R}:=$ $L_{-}(p, R / 4, R / 2): X \rightarrow[0,1]$ and $\varphi_{R}^{\alpha}:=L_{-}\left(p_{\alpha}, R / 4, R / 2\right): X_{\alpha} \rightarrow[0,1]$ for $R>0$ and $\alpha \in \mathcal{A}$.

Claim 5.4. We have the following:

(1) $\lim _{R / \infty}\left\|\varphi_{R} u-u\right\|_{L^{2}}=0$.

(2) $\underline{\lim }_{R / \infty} \mathcal{E}\left(\varphi_{R} u\right) \geq \mathcal{E}(u)$.

(3) $\left\|\varphi_{R}^{\alpha} u_{\alpha}\right\|_{L^{2}} \leq\left\|u_{\alpha}\right\|_{L^{2}}$ for any $R>0$ and $\alpha \in \mathcal{A}$. 
(4) $\mathcal{E}_{\alpha}\left(\varphi_{R}^{\alpha} u_{\alpha}\right)^{1 / 2} \leq \mathcal{E}_{\alpha}\left(u_{\alpha}\right)^{1 / 2}+4\left\|u_{\alpha}\right\|_{L^{2}} / R$ for any $R>0$ and $\alpha \in \mathcal{A}$.

(5) $\lim _{\alpha}\left\|\Phi_{\alpha} \varphi_{R}-\varphi_{R}^{\alpha}\right\|_{L^{\infty}}=0$ for any $R>0$.

(6) If $L^{2}\left(X_{\alpha} ; m\right) \ni v_{\alpha} \rightarrow v \in L^{2}(X ; m) L^{2}$ strongly, then $\varphi_{R}^{\alpha} v_{\alpha} \rightarrow \varphi_{R} v$ $L^{2}$ strongly for any $R>0$.

(7) $\varphi_{R}^{\alpha} u_{\alpha} \rightarrow \varphi_{R} u L^{2}$ weakly for any $R>0$.

Proof. The proofs of (1) and (3) are easy and omitted. (2) follows from (1) and the closedness of $\mathcal{E}$ (see Lemma 2.11). (4) follows from Lemma 5.1 and $d \Gamma\left(\varphi_{R}\right) \leq 16 d m / R^{2}$ (see Lemma 1 of [52]).

(5): Since $\left|d\left(p_{\alpha}, x\right)-d\left(p, f_{\alpha}(x)\right)\right|<\epsilon_{\alpha}$ for any $x \in B\left(p_{\alpha}, t_{\alpha}\right)$, we have $\left|\varphi_{R} \circ f_{\alpha}-\varphi_{R}^{\alpha}\right| \leq 4 \epsilon_{\alpha} / R$. This implies (5).

(6): Assume that $L^{2}\left(X_{\alpha} ; m\right) \ni v_{\alpha} \rightarrow v \in L^{2}(X ; m) L^{2}$ strongly. Then, there exists a net of functions $\tilde{v}_{\beta} \in C_{0}(X)$ such that $\tilde{v}_{\beta} \rightarrow v$ in $L^{2}(X ; m)$ and

$$
\lim _{\beta} \varlimsup_{\alpha}\left\|v_{\alpha}-\Phi_{\alpha} \tilde{v}_{\beta}\right\|_{L^{2}}=0
$$

Since $\varphi_{R} \tilde{v}_{\beta} \rightarrow \varphi_{R} v$ in $L^{2}(X ; m)$, it suffices to prove that

$$
\lim _{\beta} \varlimsup_{\alpha}\left\|\varphi_{R}^{\alpha} v_{\alpha}-\Phi_{\alpha}\left(\varphi_{R} \tilde{v}_{\beta}\right)\right\|_{L^{2}}=0
$$

In fact, the left hand side is

$$
\begin{aligned}
& \leq \lim _{\beta} \varlimsup_{\alpha}\left(\left\|\varphi_{R}^{\alpha} v_{\alpha}-\varphi_{R}^{\alpha} \Phi_{\alpha} \tilde{v}_{\beta}\right\|_{L^{2}}+\left\|\varphi_{R}^{\alpha} \Phi_{\alpha} \tilde{v}_{\beta}-\Phi_{\alpha}\left(\varphi_{R} \tilde{v}_{\beta}\right)\right\|_{L^{2}}\right) \\
& \leq \lim _{\beta} \varlimsup_{\alpha}\left(\left\|\varphi_{R}^{\alpha}\right\|_{L^{\infty}}\left\|v_{\alpha}-\Phi_{\alpha} \tilde{v}_{\beta}\right\|_{L^{2}}+\left\|\varphi_{R}^{\alpha}-\Phi_{\alpha} \varphi_{R}\right\|_{L^{\infty}}\left\|\Phi_{\alpha} \tilde{v}_{\beta}\right\|_{L^{2}}\right) \\
& =0
\end{aligned}
$$

(7): Take any functions $v_{\alpha} \in L^{2}\left(X_{\alpha} ; m_{\alpha}\right)$ and $v \in L^{2}(X ; m)$ such that $v_{\alpha} \rightarrow v L^{2}$ strongly. Since (6) implies $\varphi_{R}^{\alpha} v_{\alpha} \rightarrow \varphi_{R} v L^{2}$ strongly, we have

$$
\left(\varphi_{R}^{\alpha} u_{\alpha}, v_{\alpha}\right)_{L^{2}}=\left(u_{\alpha}, \varphi_{R}^{\alpha} v_{\alpha}\right)_{L^{2}} \rightarrow\left(u, \varphi_{R} v\right)_{L^{2}}=\left(\varphi_{R} u, v\right)_{L^{2}}
$$

for any fixed $R>0$. This completes the proof of Claim 5.4.

By Claim 5.4(3)(4), $\mathcal{E}_{\alpha, 1}\left(\varphi_{R}^{\alpha} u_{\alpha}\right)$ is uniformly bounded for all $\alpha \in \mathcal{A}$ and $R \geq 1$. Therefore, the compact convergence $\mathcal{E}_{\alpha}^{R} \rightarrow \mathcal{E}^{R}, \alpha \in \mathcal{A}_{R}$, leads to the existence of a subnet $\left\{\varphi_{R}^{\alpha} u_{\alpha}\right\}_{\alpha \in \mathcal{A}_{R}^{\prime}}$ of $\left\{\varphi_{R}^{\alpha} u_{\alpha}\right\}_{\alpha \in \mathcal{A}_{R}}$ which $L^{2}$ strongly 
converges to a function $v \in L^{2}(X ; m)$. Claim 5.4(7) implies $v=\varphi_{R} u$. By Lemma 5.6, (F1) for $\mathcal{E}_{\alpha}^{R} \rightarrow \mathcal{E}^{R}$, and Claim 5.4(4), we have

$$
\mathcal{E}\left(\varphi_{R} u\right) \leq \varliminf_{\alpha \in \mathcal{A}_{R}^{\prime}} \mathcal{E}_{\alpha}\left(\varphi_{R}^{\alpha} u_{\alpha}\right) \leq \varliminf_{\alpha \in \mathcal{A}_{R}^{\prime}}\left(\mathcal{E}_{\alpha}\left(u_{\alpha}\right)^{1 / 2}+4\left\|u_{\alpha}\right\|_{L^{2}} / R\right)^{2} .
$$

From this and Claim 5.4(2),

$$
\mathcal{E}(u) \leq \lim _{R \nearrow \infty} \mathcal{E}\left(\varphi_{R} u\right) \leq \varlimsup_{\alpha \in \mathcal{A}} \mathcal{E}_{\alpha}\left(u_{\alpha}\right)
$$

This completes the proof of Theorem 5.2.

\subsection{Convergence of (noncompact) manifolds under a lower bound of Ricci curvature.}

In this section, we shall prove Theorem 1.3 as an application of Theorem 5.2 .

Let $\mathcal{P} \mathcal{R}(n, c)$ be as defined in $\S 1$. Recall that $\mathcal{S}\left(L^{2}\left(\mathcal{M}_{c}\right)\right)$ is the set of spectral structures on $L^{2}(X ; m)$, where $(X, p, m) \in \mathcal{M}_{c}$ runs over all spaces (see Definitions 2.3 and 2.14). We first prove:

Lemma 5.7. The closure of $\left\{\Sigma\left(M, \mu_{p}\right) \mid\left(M, p, \mu_{p}\right) \in \mathcal{P} \mathcal{R}(n, D)\right\}$ is sequentially compact in $\mathcal{S}\left(L^{2}\left(\mathcal{M}_{c}\right)\right.$ ) with respect to the strong spectral topology.

Proof. Since any net in $\mathcal{P} \mathcal{R}(n, c)$ satisfies $(\mathrm{N}),(\mathrm{M}),(\mathrm{C})$, and $(\mathrm{P})($ see $[28,9])$, Theorem 5.2 implies the theorem.

Assume from now on that a net $\left\{\left(M_{\alpha}, p_{\alpha}, \mu_{p_{\alpha}}\right)\right\}_{\alpha \in \mathcal{A}} \subset \mathcal{P} \mathcal{R}(n, c)$ converges to a space $(X, p, m) \in \mathcal{M}_{c}$ with respect to the measured $\mathrm{GH}$ topology. We set $\mathcal{E}_{\alpha}:=\mathcal{E}_{\left(M_{\alpha}, \mu_{p_{\alpha}}\right)}$ and $\mathcal{E}:=\mathcal{E}_{(X, m)}$ for simplicity.

Lemma 5.8. Let $R>0$ be a fixed number. Denote by $\lambda_{k}\left(\right.$ resp. $\left.\lambda_{k}^{\alpha}\right)$ the $k^{\text {th }}$ eigenvalue of the Laplacian on $B(p, R)$ (resp. $B\left(p_{\alpha}, R\right)$ ) with Dirichlet boundary condition and with multiplicity. Then we have $\lambda_{k}^{\alpha} \rightarrow \lambda_{k}$ for each $k$. Moreover, for any orthonormal basis $\left\{\varphi_{k}\right\}_{k=1,2, \ldots}$ on $L^{2}(B(p, R) ; m)$ consisting of eigenfunctions for $\left\{\lambda_{k}\right\}$, there exists an orthonormal basis $\left\{\varphi_{k}^{\alpha}\right\}$ on $L^{2}\left(B\left(p_{\alpha}, R\right) ; \mu_{p_{\alpha}}\right)$ consisting of eigenfunctions for $\left\{\lambda_{k}^{\alpha}\right\}$ such that $\varphi_{k}^{\alpha} \rightarrow \varphi_{k}$ $L^{2}$ strongly for each $k$.

Proof. The lemma follows basically from the same discussion as in $\S 7$ of [16]. An essential part of the proof is the following: 
Claim 5.5. (1) For any $u \in \mathcal{D}(\mathcal{E}) \cap C_{0}(B(p, R))$ there exists a net $u_{\alpha} \in$ $W_{0}^{1,2}\left(B\left(p_{\alpha}, R\right) ; \mu_{p}\right)$ such that $u_{\alpha} \rightarrow u L^{2}$ strongly and

$$
\mathcal{E}(u) \geq \varlimsup_{\alpha} \mathcal{E}_{\alpha}\left(u_{\alpha}\right)
$$

(2) For any net of Dirichlet eigenfunctions $u_{\alpha}$ on $B(p, R)$ of the Laplacian with eigenvalues $\lambda_{\alpha}$ with $\sup _{\alpha} \lambda_{\alpha}<\infty$ and $\left\|u_{\alpha}\right\|_{L^{2}}=1$, there exists a subnet of $\left\{u_{\alpha}\right\}$ which $L^{2}$ strongly converges to some function $u$ such that

$$
\mathcal{E}(u) \leq \varliminf_{\alpha} \mathcal{E}_{\alpha}\left(u_{\alpha}\right)
$$

Proof. (1) follows from Lemma 10.7 of [15].

We will prove $(2)$. By Theorem 5.3, $\left\{u_{\alpha}\right\}$ has an $L^{2}$ strong convergent subnet. Without loss of generality, we may assume that $u_{\alpha} L^{2}$ strongly converges to a function $u \in L^{2}(B(p, R))$. Take any $r \in(0, R)$ and fix it. Then, by Li-Yau's gradient estimate [40], we obtain the uniform bound of the norm of the gradient of $u_{\alpha}$ on $B\left(p_{\alpha}, r\right)$ (see also [31]). We also have the uniform bound of $\left\|\nabla\left|\nabla u_{\alpha}\right|\right\|_{L^{2}}$ from the Weitzenböck formula and the lower bound of Ricci curvature. Thus, Proposition 16.43 of [15] (see also Lemma 16.39 of [15]) shows that, taking $\epsilon_{\alpha}$-approximations $f_{\alpha}: B(p, r) \rightarrow B\left(p_{\alpha}, r\right)$ with $\epsilon_{\alpha} \searrow 0$, i.e.,

$$
\begin{gathered}
\left|d_{\alpha}\left(f_{\alpha}(x), f_{\alpha}(y)\right)-d(x, y)\right|<\epsilon_{\alpha} \quad \text { for any } x, y \in B(p, r), \\
B\left(p_{\alpha}, r\right) \subset B\left(f_{\alpha}(B(p, r)), \epsilon_{\alpha}\right)
\end{gathered}
$$

the function $\hat{u}_{\alpha}:=u_{\alpha} \circ f_{\alpha} L^{2}$ converges to a function, say $u_{r}$, on $B(p, r)$ such that

$$
\mathcal{E}\left(u_{r}\right) \leq \underline{\lim _{\alpha}} \mathcal{E}_{\alpha}\left(\left.u_{\alpha}\right|_{B\left(p_{\alpha}, r\right)}\right) \leq \underline{\lim _{\alpha}} \mathcal{E}_{\alpha}\left(u_{\alpha}\right) .
$$

Since $u_{r}=\left.u\right|_{B(p, r)}$, we obtain (2).

Once we obtain the claim above, Lemma 5.8 follows from a standard inductive argument (a simplified version of the proof of Theorem 2.6).

Lemma 5.9. $\mathcal{E}_{\alpha} \Gamma$-converges to $\mathcal{E}$.

Proof. We will check (F1). Assume that a net $u_{\alpha} \in L^{2}\left(M_{\alpha} ; \mu_{p_{\alpha}}\right) L^{2}$ strongly converges to a function $u \in L^{2}(X ; m)$, and that $\sup _{\alpha} \mathcal{E}_{\alpha}\left(u_{\alpha}\right)<\infty$. Since 
$\mathcal{D}(\mathcal{E}) \cap C_{0}(X)$ is dense in $\left(\mathcal{D}(\mathcal{E}), \mathcal{E}_{1}^{1 / 2}\right.$ ) (see [16]), for any $\epsilon>0$ there is a function $\tilde{u}$ on $X$ with compact support such that $\mathcal{E}_{1}(u-\tilde{u})<\epsilon$. Taking a number $R>0$ with supp $\tilde{u} \subset B(p, R)$, we set, for $k, N \in \mathbb{N}$,

$$
\begin{aligned}
& c_{k}:=\left(u, \varphi_{k}\right)_{L^{2}}, \quad \tilde{c}_{k}:=\left(\tilde{u}, \varphi_{k}\right)_{L^{2}}, \quad c_{k}^{\alpha}:=\left(u_{\alpha}, \varphi_{k}\right)_{L^{2}}, \\
& u^{(N)}:=\sum_{k=1}^{N} c_{k} \varphi_{k}, \quad \tilde{u}^{(N)}:=\sum_{k=1}^{N} \tilde{c}_{k} \varphi_{k}, \quad u_{\alpha}^{(N)}:=\sum_{k=1}^{N} c_{k}^{\alpha} \varphi_{k}^{\alpha},
\end{aligned}
$$

where $\varphi_{k}, \varphi_{k}^{\alpha}$ are as in Lemma 5.8. Then we have for each $k \in \mathbb{N}$,

$$
\left|c_{k}-\tilde{c}_{k}\right|<\epsilon \text { and } \quad \lim _{\alpha} c_{k}^{\alpha}=c_{k}
$$

Since for any $N \in \mathbb{N}$,

$$
\mathcal{E}_{\alpha}\left(u_{\alpha}\right) \geq \mathcal{E}_{\alpha}\left(\left.u_{\alpha}\right|_{B\left(p_{\alpha}, R\right)}\right) \geq \mathcal{E}\left(u_{\alpha}^{(N)}\right)=\sum_{k=1}^{N} \lambda_{k}^{\alpha}\left(c_{k}^{\alpha}\right)^{2},
$$

Lemma 5.8 shows

$$
\varliminf_{\alpha} \mathcal{E}_{\alpha}\left(u_{\alpha}\right) \geq \sum_{k=1}^{N} \lambda_{k} c_{k}^{2} \geq(1-\theta(\epsilon)) \sum_{k=1}^{N} \lambda_{k} \tilde{c}_{k}^{2}=(1-\theta(\epsilon)) \mathcal{E}\left(\tilde{u}^{(N)}\right) .
$$

Letting $N \rightarrow \infty$ yields

$$
\varliminf_{\alpha} \mathcal{E}_{\alpha}\left(u_{\alpha}\right) \geq(1-\theta(\epsilon)) \mathcal{E}(\tilde{u}) \geq(1-\theta(\epsilon))(\mathcal{E}(u)-\theta(\epsilon)) .
$$

This completes the proof of (F1).

(F2) is implied by Claim 5.5(1) and (F1).

We restate Theorem 1.3.

Theorem 5.4. Let a net $\left\{\left(M_{\alpha}, p_{\alpha}, \mu_{p_{\alpha}}\right)\right\}_{\alpha \in \mathcal{A}} \subset \mathcal{P} \mathcal{R}(n, c)$ converge to a space $(X, p, m) \in \mathcal{M}_{c}$ with respect to the measured $G H$ topology. Then, the spectral structure $\Sigma\left(M_{\alpha}, \mu_{p_{\alpha}}\right)$ strongly converges to $\Sigma(X, m)$. In particular we have

$$
\sigma(\Delta) \subset \lim _{\alpha} \sigma\left(\Delta_{\alpha}\right)
$$

where $\Delta, \Delta_{\alpha}$ are the generators of $\Sigma(X, m), \Sigma\left(M_{\alpha}, \mu_{p_{\alpha}}\right)$ respectively. 
Proof. It suffices to prove that $\mathcal{E}_{\alpha}:=\mathcal{E}_{\left(M_{\alpha}, \mu_{p_{\alpha}}\right)}$ Mosco converges to $\mathcal{E}:=$ $\mathcal{E}_{(X, m)}$. Suppose the contrary, so that there exists a subnet $\mathcal{B} \subset \mathcal{A}$ and a neighborhood $U$ of $\mathcal{E}$ in the space $\mathcal{F}\left(L^{2}\left(\mathcal{M}_{c}\right)\right.$ ) of closed bilinear forms equipped with the Mosco topology such that $\mathcal{E}_{\beta}$ for any $\beta \in \mathcal{B}$ is not contained in $U$. Lemma 5.7 implies the existence of a Mosco convergent subnet of $\left\{\mathcal{E}_{\beta}\right\}_{\beta \in \mathcal{B}}$, the limit of which must coincide with the $\Gamma$-limit $\mathcal{E}$ (see Lemma 5.9). This is a contradiction and the proof is completed.

Remark 5.1. For $n \geq 2$ and $D>0$, let $\mathcal{R}(n, D)$ be the set of $\left(M, \mu_{M}\right)$, where $M$ is any closed Riemannian manifold of $\operatorname{Ric}_{M} \geq-(n-1)$ and $\operatorname{diam} M \leq D$ and where $\mu_{M}:=\operatorname{vol}_{M} / \operatorname{vol}(M)$. Combining the works of Kasue-Kumura [32] and Cheeger-Colding [16] yields that the topology induced from the spectral distance coincides with the measured GH topology on the measured GH closure of $\mathcal{R}(n, D)$. Note that the topology induced from the spectral distance is stronger than the compact spectral topology in general. In particular, if $X$ is compact in Theorem 5.4, the conclusion 'strongly converges' can be replaced with 'compactly converges'.

\section{References.}

[1] S. Albeverio, S. Kusuoka, and L. Streit, Convergence of Dirichlet forms and associated Schrödinger operators, J. Funct. Anal. 68 (1986), no. 2, 130-148.

[2] C. Anné, Fonctions propres sur des variétés avec des anses fines, application à la multiplicité, Séminaire de Théorie Spectrale et Géométrie, No. 7, Année 1988-1989, Univ. Grenoble I, Saint, 1989, pp. 123-133.

[3] — Formes différentielles sur des variétés avec des anses fines, Actes de la Table Ronde de Géométrie Différentielle (Luminy, 1992), Soc. Math. France, Paris, 1996, pp. 69-76.

[4] C. Anné and B. Colbois, Opérateur de Hodge-Laplace sur des variétés compactes privées d'un nombre fini de boules, J. Funct. Anal. 115 (1993), no. 1, 190-211.

[5] , Spectre du laplacien agissant sur les p-formes différentielles et écrasement d'anses, Math. Ann. 303 (1995), no. 3, $545-573$.

[6] W. Ballmann, M. Gromov, and V. Schroeder, Manifolds of nonpositive curvature, Birkhäuser Boston Inc., Boston, Mass., 1985. 
[7] P. Bérard, G. Besson, and S. Gallot, Embedding Riemannian manifolds by their heat kernel, Geom. Funct. Anal. 4 (1994), no. 4, 373-398.

[8] Yu. Burago, M. Gromov, and G. Perel'man, A. D. Aleksandrov spaces with curvatures bounded below, Uspekhi Mat. Nauk 47 (1992), no. 2(284), 3-51, 222, translation in Russian Math. Surveys 47 (1992), no. $2,1-58$.

[9] P. Buser, A note on the isoperimetric constant, Ann. Sci. École Norm. Sup. (4) 15 (1982), no. 2, 213-230.

[10] I. Chavel and E. A. Feldman, Spectra of domains in compact manifolds, J. Funct. Anal. 30 (1978), no. 2, 198-222.

[11] , Spectra of manifolds with small handles, Comment. Math. Helv. 56 (1981), no. 1, 83-102.

[12] Isoperimetric constants of manifolds with small handles, Math. Z. 184 (1983), no. 4, 435-448.

[13] Diffusion on manifolds with small handles, Indiana Univ. Math. J. 34 (1985), no. 3, 449-461.

[14] J. Cheeger, A lower bound for the smallest eigenvalue of the Laplacian, Problems in analysis (Papers dedicated to Salomon Bochner, 1969), Princeton Univ. Press, Princeton, N. J., 1970, pp. 195-199.

[15] Differentiability of Lipschitz functions on metric measure spaces, Geom. Funct. Anal. 9 (1999), no. 3, 428-517.

[16] J. Cheeger and T. H. Colding, On the structure of spaces with Ricci curvature bounded below. III, J. Differential Geom. 54 (2000), no. 1, $37-74$.

[17] B. Colbois and G. Courtois, Convergence de variétés et convergence du spectre du Laplacien, Ann. Sci. École Norm. Sup. (4) 24 (1991), no. 4, 507-518.

[18] G. Dal Maso, An introduction to $\Gamma$-convergence, Birkhäuser Boston Inc., Boston, MA, 1993.

[19] G. Dal Maso, R. Gulliver, and U. Mosco, Asymptotic spectrum of manifolds of increasing topological type, preprint, 1999. 
[20] G. De Cecco and G. Palmieri, Intrinsic distance on a Lipschitz Riemannian manifold, Rend. Sem. Mat. Univ. Politec. Torino 46 (1988), no. 2, 157-170 (1990).

[21] L. C. Evans and R. F. Gariepy, Measure theory and fine properties of functions, Studies in Advanced Mathematics, CRC Press, Boca Raton, FL, 1992.

[22] K. Fujiwara, Convergence of the eigenvalues of Laplacians in a class of finite graphs, Contemp. Math. 173 (1994), 115-120.

[23] K. Fukaya, Collapsing of Riemannian manifolds and eigenvalues of Laplace operator, Invent. Math. 87 (1987), 517-547.

[24] M. Fukushima, Y. Oshima, and M. Takeda, Dirichlet forms and symmetric Markov processes, de Gruyter Studies in Math., vol. 19, Walter de Gruyter, Berlin-New York, 1994.

[25] M. P. Gaffney, The harmonic operator for exterior differential forms, Proc. Nat. Acad. Sci. U. S. A. 37 (1951), 48-50.

[26] — A special Stokes's theorem for complete Riemannian manifolds, Ann. of Math. (2) 60 (1954), 140-145.

[27] Hilbert space methods in the theory of harmonic integrals, Trans. Amer. Math. Soc. 78 (1955), 426-444.

[28] M. Gromov, Metric structures for Riemannian and non-Riemannian spaces, Birkhäuser Boston Inc., Boston, MA, 1999, Based on the 1981 French original [MR 85e:53051], With appendices by M. Katz, P. Pansu and S. Semmes, Translated from the French by Sean Michael Bates.

[29] N. Ikeda and Y. Ogura, A degenerating sequence of Riemannian metrics on a manifold and their Brownian motions, Diffusion processes and related problems in analysis, Vol. I (Evanston, IL, 1989), Birkhäuser Boston, Boston, MA, 1990, pp. 293-312.

[30] T. Kanahori, Convergence of the eigenvalues of Laplacians of degenerating sequence of finite graphs, preprint, 1998.

[31] A. Kasue, Convergence of Riemannian manifold and Laplace operators. I, II, preprint, 1999 .

[32] A. Kasue and H. Kumura, Spectral convergence of Riemannian manifolds, Tôhoku Math. J. (2) 46 (1994), no. 2, 147-179. 
[33] ․ Spectral convergence of Riemannian manifolds. II, Tôhoku Math. J. (2) 48 (1996), no. 1, 71-120.

[34] Spectral convergence of conformally immersed surfaces with bounded mean curvature, J. Geom. Anal. 12 (2002), no. 4, 663681.

[35] A. Kasue, H. Kumura, and Y. Ogura, Convergence of heat kernels on a compact manifold, Kyushu J. Math. 51 (1997), no. 2, 453-524.

[36] T. Kato, Perturbation theory for linear operators, second ed., SpringerVerlag, Berlin, 1976, Grundlehren der Mathematischen Wissenschaften, Band 132.

[37] K. Kuwae, Y. Machigashira, and T. Shioya, Beginning of analysis on Alexandrov spaces, Geometry and topology: Aarhus (1998), Amer. Math. Soc., Providence, RI, 2000, pp. 275-284.

[38] Sobolev spaces, Laplacian, and heat kernel on Alexandrov spaces, Math. Z. 238 (2001), no. 2, 269-316.

[39] P. Li and G. Tian, On the heat kernel of the Bergmann metric on algebraic varieties, J. Amer. Math. Soc. 8 (1995), no. 4, 857-877.

[40] P. Li and S. T. Yau, Estimates of eigenvalues of a compact Riemannian manifold, Geometry of the Laplace operator (Proc. Sympos. Pure Math., Univ. Hawaii, Honolulu, Hawaii, 1979), Amer. Math. Soc., Providence, R.I., 1980, pp. 205-239.

[41] J. Masamune, Essential self-adjointness of Laplacians on Riemannian manifolds with fractal boundary, Comm. Partial Differential Equations 24 (1999), no. 3-4, 749-757.

[42] U. Mosco, Composite media and asymptotic Dirichlet forms, J. Funct. Anal. 123 (1994), no. 2, 368-421.

[43] J. R. Norris, Heat kernel asymptotics and the distance function in Lipschitz Riemannian manifolds, Acta Math. 179 (1997), 79-103.

[44] L. Notarantonio, Spectrum of compact manifolds with high genus, preprint.

[45] Y. Otsu and T. Shioya, The Riemannian structure of Alexandrov spaces, J. Differential Geom. 39 (1994), no. 3, 629-658. 
[46] G. Perelman, A. D. Alexandrov's spaces with curvatures bounded from below II, preprint.

[47] M. Reed and B. Simon, Methods of modern mathematical physics, vol. I - IV, Academic Press, 1975.

[48] I. E. Segal and R. A. Kunze, Integrals and operators, enlarged ed., Springer-Verlag, Berlin, 1978, Grundlehren der Mathematischen Wissenschaften, Band 228.

[49] T. Shioya, Mass of rays in Alexandrov spaces of nonnegative curvature, Comment. Math. Helv. 69 (1994), no. 2, 208-228.

[50] The limit spaces of two-dimensional manifolds with uniformly bounded integral curvature, Trans. Amer. Math. Soc. 351 (1999), no. $5,1765-1801$.

[51] Convergence of Alexandrov spaces and spectrum of Laplacian, J. Math. Soc. Japan 53 (2001), no. 1, 1-15.

[52] K.-T. Sturm, Analysis on local Dirichlet spaces. I. Recurrence, conservativeness and $L^{p}$-Liouville properties, J. Reine Angew. Math. 456 (1994), 173-196.

[53] On the geometry defined by Dirichlet forms, Seminar on Stochastic Analysis, Random Fields and Applications (Ascona, 1993), Progr. Probab., vol. 36, Birkhäuser, Basel, 1995, pp. 231-242.

[54] J. Takahashi, Collapsing of connected sums and the eigenvalues of the Laplacian, J. Geom. Phys. 40 (2002), no. 3-4, 201-208.

[55] N. Teleman, The index of signature operators on Lipschitz manifolds, Inst. Hautes Études Sci. Publ. Math. (1983), no. 58, 39-78 (1984).

[56] S. A. Wolpert, Spectral limits for hyperbolic surfaces. I, II, Invent. Math. 108 (1992), no. 1, 67-89, 91-129.

[57] S. T. Yau, Isoperimetric constants and the first eigenvalue of a compact Riemannian manifold, Ann. Sci. École Norm. Sup. (4) 8 (1975), no. 4, 487-507.

[58] K. Yosida, Functional analysis, sixth ed., Springer-Verlag, Berlin, 1980. 
KAZUHIRO KuWAE

Department of Mathematical Sciences

Yokohama City University

YOKOHAMA 236-0027, JAPAN

kuwae@math.yokohama-cu.ac.jp

TAKASHI SHIOYA

Mathematical Institute

TÔHOKU UNIVERSITY

SENDAI 980-8578, JAPAN

shioya@math.tohoku.ac.jp

Received June 7, 2001. 